\title{
PHYTOSOCIOLOGICAL STUDY OF BEECH AND BEECH-MIXED WOODS IN MONTI SIBILLINI NATIONAL PARK (CENTRAL APENNINES, ITALY)
}

\author{
Andrea CATORCI ${ }^{1}$, Alessandra VITANZI ${ }^{2} \&$ Sandro BALLELLI ${ }^{1}$
}

\begin{abstract}
The results of a phytosociological survey of the woods mainly growing on limestones in the Southern part of the Marches Region (Central Italy), that is part of the Monti Sibillini National Park, are here presented. This area is characterized by a temperate climate (upper mesotemperate and lower/upper supratemperate bioclimatic belts). In order to study the vegetation, 173 phytosociological relevés were carried out through the Braun-Blanquet methodology. All the data obtained were submitted to multivariate analysis. The phytosociological analysis is characterized in nine associations, whereof three are of new description (Lathyro veneti-Fagetum sylvaticae hieracietosum murorum, Cardamino kitaibelii-Fagetum sylvaticae corallorhizetosum trifidae and Cardamino kitaibelii-Fagetum sylvaticae anemonetosum nemorosae).

Key words: beech woods, calcareous substratum, Central Apennines, Monti Sibillini, multivariate analysis, phytosociology.

\section{Izvleček}

Prikazani so rezultati fitocenološke raziskave gozdov, ki uspevajo predvsem na apnencu v južnem delu pokrajine Marche (srednja Italija), to je v delu Nacionalnega parka Monti Sibillini. To območje označuje zmerna klima (zgornji mezotemperatni in spodnji/zgornji supratemperatni bioklimatki pas). Vegetacijo smo proučili s 173 fitocenološkimi popisi po Braun-Blanquetovi metodi. Vse popisno gradivosmo analizirali z multivariatno statistiko. S fitocenološko analizo smo dobili devet asociacij, od tega so tri novoopisane (Lathyro veneti-Fagetum sylvaticae hieracietosum murorum, Cardamino kitaibelii-Fagetum sylvaticae corallorhizetosum trifidae and Cardamino kitaibelii-Fagetum sylvaticae anemonetosum nemorosae).

Ključne besede: bukovi gozdovi, apnenčasti substrat, Srednji Apenini, Monti Sibillini, multivariatna analiza, fitosociologija.
\end{abstract}

\section{INTRODUCTION}

Italian beech woods develop on mountain relief belonging to the biogeographical Alpine and Apennine-Balcanic Provinces (Eurosiberian Region) and to the Italian-Tyrrhenian and Adriatic Provinces (Mediterranean Region) (Rivas-Martínez et al. 2004) as highlighted in Figure 1, at altitudes ranging from $(200-300 \mathrm{~m}) 600$ to $2000 \mathrm{~m}$ a.s.l. (Pignatti 1982, Scoppola \& Caporali 1998).

According to Biondi et al. (2002), Italian beech woodlands are placed into two alliances:
Aremonio-Fagion sylvaticae, widespread on the Alpine ridge and in the Central and Northern Apennines (Credano et al. 1980, Ubaldi \& Speranza 1985, Poldini \& Vidali 1995, Willner 2002) and Geranio versicoloris-Fagion sylvaticae, distributed in the Central and Southern sector of the Italian Peninsula (Bonin 1967-69, Gentile 1970, Brullo 1983, Di Pietro 2002, Pirone et al. 2003, Biondi et al. 2004, Di Pietro et al. 2004, Taffetani et al. 2004, Blasi et al. 2005, Pirone et al. 2005, Rosati et al. 2005, Ciaschetti et al. 2006, Di Pietro 2007, Allegrezza \& Biondi 2008, Biondi et al. 2008).

\footnotetext{
${ }^{1}$ School of Environmental Sciences, UNICAM Università di Camerino, via Pontoni 5, I-62032 Camerino (MC); e-mail: andrea.catorci@unicam.it, sandro.ballelli@unicam.it

${ }^{2} \mathrm{PhD}$ in Environmental Sciences and Public Health, UNICAM Università di Camerino; e-mail: alessandra.vitanzia) unicam.it
} 


\section{B. EUROSIBERIAN}

Bc. ALPINO-CAUCASIAN

8. Alpine

8a. Mediterranean Alpine, 8b Western Alpine, 8c. Central Alpine

8d. Eastern Alpine

9. Apennino-Balkan

9a. Apennine, 9b. Padanian, 9c. Ilyrian, 9d. Pindan, 9e. Bulgarian

C. MEDITERRANEAN

Ca. WESTERN MEDITERRANEAN

20. Italo-Thyrrhenian

20a. Corsican, 20b. Sardinian, 20c Sicilian, 20d. Coastal West Italian

Cb. EASTERN MEDITERRANEAN

21. Adriatic

21a. Apulian, 21b. Epiro-Dalmatian 21c. Peloponnesian

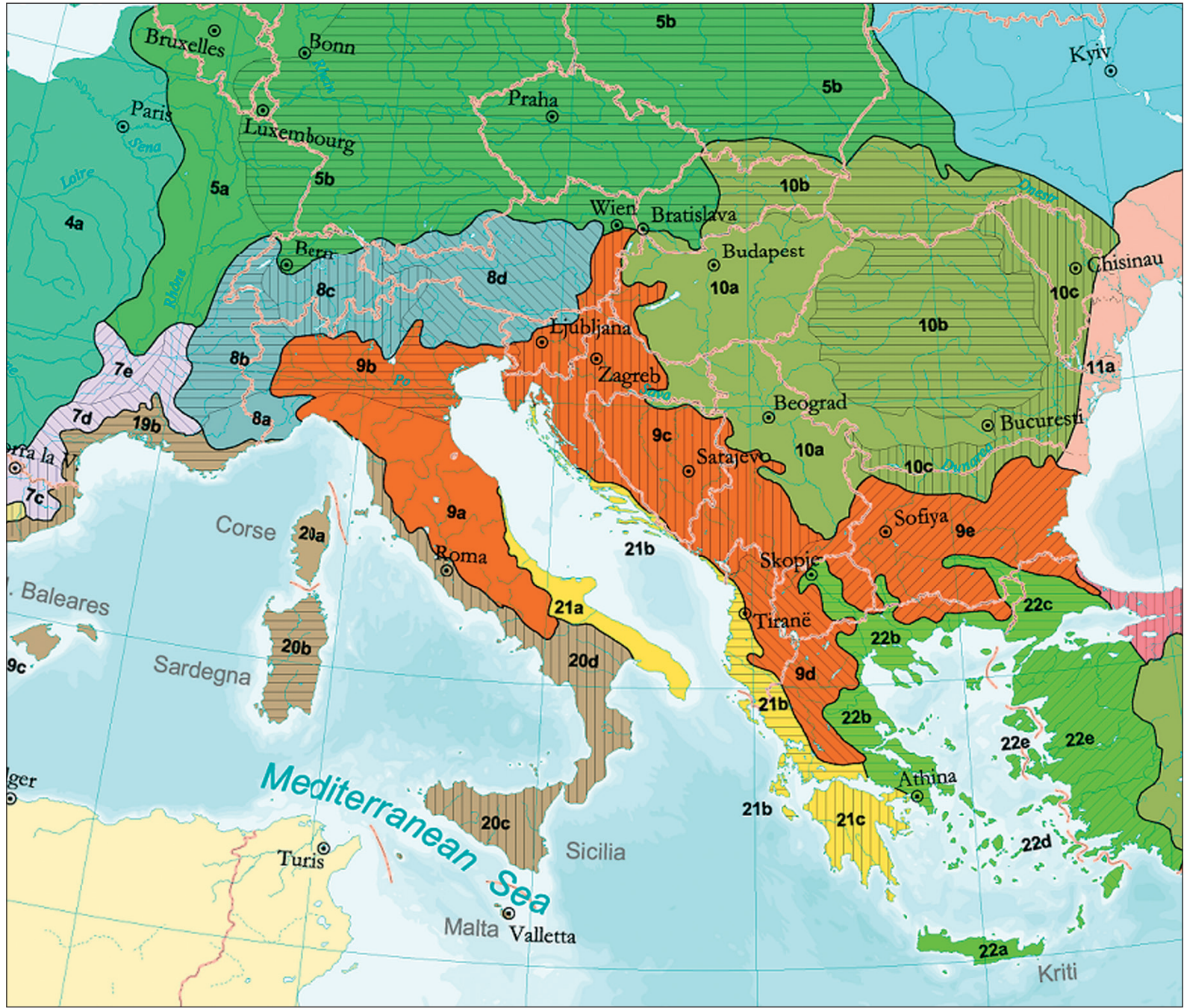

Figure 1: Part of Biogeographic map of Europe (Rivas-Martínez et al. 2004). Slika 1: Izsek iz biogeografske karte Evrope (Rivas-Martínez et. al 2004). 
These two alliances overlay in correspondence to the Umbria-Marches Apennines (Central Italy). In this biogeographical sector, the beech woods, belonging to the Geranio versicoloris-Fagion sylvaticae alliance, are present in the lower supratemperate bioclimatic belt, while the ones included in Aremonio-Fagion sylvaticae alliance develop in the upper supratemperate bioclimatic belt (Biondi et al. 2002, Allegrezza 2003, Catorci et al. 2003, Biondi et al. 2004). Furthermore, mountain forest landscape of the Central Apennines includes gorge plant communities belonging to Tilio platyphylli-Acerion pseudoplatani alliance (Biondi et al. 2002, Catorci et al. 2003, Paura \& Cutini 2006).

The study of beech woods of the Monti Sibillini National Park is part of a wider research comprising the survey of flora, vegetation and plant landscape of such a protected area. Moreover, the work is aimed at completing the set of information related to the mountain forests of the Central Apennines through the analysis of a mountain sector representing the one ridge of Umbria-Marches Apennines, where the altitudes of relief (2200$2400 \mathrm{~m}$ ) rise to the ecological timberline (located at about 1800-1850 m a.s.l., Pedrotti 1995).

\section{STUDY AREA AND FOREST MANAGEMENT}

From the geological viewpoint, Monti Sibillini National Park (Central Italy - Figure 2) is mainly formed by limestones, except for the peripheral pedemontane sectors, where sandstone and mar-

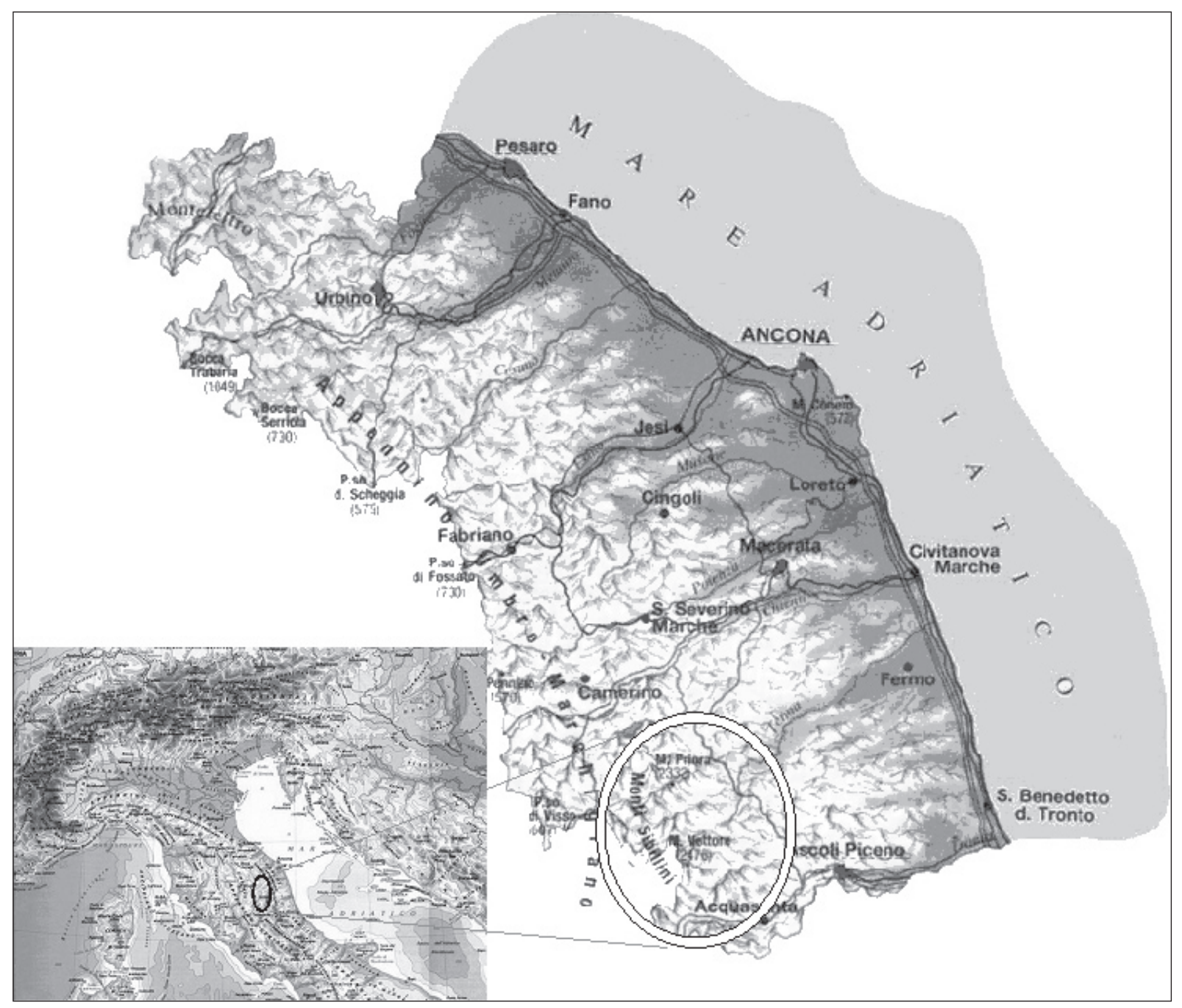

Figure 2: Study area.

Slika 2: Raziskovano območje. 
ly-limestone substrata outcrop (AA.vv. 1991). The lowest altitude is about $400 \mathrm{~m}$, while the highest is $2476 \mathrm{~m}$ (Monte Vettore).

The bedrock's geochemical features and glacial or post-glacial erosion processes have contributed to the formation of rough morphologies with extremely steep slopes carved by gorges, valleys and rocky walls. Such geomorphological and historicalclimatic features as well as the past land use have influenced a very slowly pedogenesis, also favouring the erosion of the most superficial soil layer.

The most widespread soil type in beech woods growing on limestones is Skeleti-Calcaric Phaeozem alternated by Calcari-Mollihumic Leptosol (A.S.S.A.M. 2006). These soils are moderately deep (about $0.5-1.5 \mathrm{~m}$ ), with quite a fine texture and changeable amount of skeleton. Organic matter content in shallow horizons is high or very high, the reaction is slightly basic, while cationic exchange capacity and base saturation are high (Giovagnotti et al. 2003, Calandra \& Leccese 2007). Soils of beech woods, growing on sandstones, are mostly Calcaric Cambisols and secondarily Calcaric Regosols; these soil types are characterized by strong desaturation, low Active Calcium content, $\mathrm{pH}$ lower than 6, sandy or sandy-clayey texture and moderate to mean depth (I.P.L.A. 2001, A.S.S.A.M. 2006).

In the study area, woodlands dominated by $\mathrm{Fa}$ gus sylvatica subsp. sylvatica are developing from 900-950 m to 1650-1700 (1750) m a.s.l., in correspondence to upper mesotemperate, lower and upper supratemperate bioclimatic belts (Table 1) (Biondi et al. 1995, Catorci et al. 2007). In impluvia of North-facing slopes, the lower limit falls down to $700-750 \mathrm{~m}$, while on watersheds and South-facing slopes, this limit rises up to about $1200 \mathrm{~m}$.

Beech woods in Monti Sibillini National Park extend for overall 8300 ha, of which about 1000 ha are part of upper mesotemperate bioclimatic belt, 5300 ha of the lower supratemperate and 1900 ha of the upper supratemperate bioclimatic belt.

In the study area, beech forests have been affected for centuries by remarkable human pressures, both for the production of firewood and for summer grazing (Sansa 2003). Nowadays, the most widespread management type of Sibillini beech woods is coppice with standard (about $80 \%$ ), with the shortest cutting turnover of 25 years and longest of 40 , while only about $20 \%$ is managed as high-forest (I.P.L.A. 2001).

However, the lack of economic interest in extensive livestock and mountain forestry in the last
30-40 years has favoured the natural expansion of woodlands as well as their generalized ageing. As a matter of fact, in many Sibillini sectors, beech woods can be considered as old coppice because they have not been cut for one or two turns (40-80 years). Current researches (Vitanzi et al. work in progress) did not highlight the presence of old forests in accordance with the criteria proposed by Franklin \& Spies (1991), Di Filippo et al. (2004) and Burrascano et al. (2009).

\section{METHODS}

The study of Monti Sibillini National Park beech woods was carried out using the phytosociological methodology of the Sigmatist Zurich-Montpellier school (Braun-Blanquet 1931, 1964), integrated with the recent acquisitions in synphytosociology and geosynphytosociology (Géhu \& Rivas-Martínez 1981, Theurillat 1992, Biondi 1996, Biondi et al. 2004, Rivas-Martínez 2005, 2005a). In particular, 173 relevés were executed from 2004 to 2008. Then, the relevés table was submitted to multivariate analysis using Syntax 2000 software (Podani 2001). Phytosociological values have been converted according to the Van der Maarel (1979) scale, obtaining a matrix made up of 222 rows (species) and 173 columns (relevés) that was submitted to numerical classification through the Group average algorithm (Orloci 1978), on a similarity matrix, based on chord distance.

Floristic nomenclature follows Tutin et al. (1964-80, 1993) and Conti et al. (2005, 2007). Publications regarding syntaxonomic review and local phytosociological study were considered in order to define the vegetation types (Allegrezza et al. 2002, Biondi et al. 2002, 2004, Allegrezza 2003, Catorci et al. 2003, Blasi et al. 2004, Taffetani et al. 2004, Ballelli et al. 2006).

The phytosociological data obtained were submitted to different processing methods/operations:

- Principal Components Analysis (PCA), using Syntax 2000 software (Podani 2001), of a matrix made up of 4 rows (environmental parameters - geology, altitude, slope angle and morphology - expressed by classes as shown in Table 2) $\times 11$ columns (syntaxa), in order to identify the weight of the different local factors defining the forest syntaxa distribution;

- calculation of the weighted phytosociological spectrum (Tomaselli 1956) for each syntaxon; 
phytosociological spectra were carried out starting from species number and their ponderal weight inside the two orders (Quercetalia pubescenti-petraeae and Fagetalia sylvaticae) and membership class (Querco-Fagetea) represented in phytosociological tables attached to this work.

Soil data, related to the identified plant communities were obtained using: a graduated pole for measurement of depth; a field pH-meter; an electromagnetic sieve for textures. Depth, $\mathrm{pH}$ and texture values were collected from some sampling areas for each syntaxon.

\section{RESULTS}

\subsection{Phytosociological GHARAGTERIZATION}

Multivariate analysis of phytosociological relevés (Figure 3) points out two main groups: I - highhilly mixed woods (Fagus sylvatica subsp. sylvatica and Ostrya carpinifolia) belonging to Quercetalia pubescenti-petraeae order; II - mountain woods with a dominance of Fagus sylvatica subsp. sylvatica, belonging to Fagetalia sylvaticae order. Inside cluster II, two sub-clusters can be identified: the first corresponding to Geranio versicoloris-Fagion sylvaticae and Aremonio-Fagion sylvaticae alliances (sub-cluster IIa), the second belonging to Tilio plathyphylliAcerion pseudoplatani alliance (sub-cluster IIb).

The sub-clusters are further divided into two or more relevés groups corresponding to association, subassociation or variant syntaxonomical levels, which are hereafter defined from a phy- tosociological viewpoint and described as regards floristic and ecological characteristics.

As for the importance of the environmental parameters for the distribution of the surveyed forest syntaxa, the biplot in Figure 4 shows that axes 1, 2 and 3 explain respectively $47.6 \%, 31.0 \%$ and $17.6 \%$ of the total variance. This analysis highlights that the variable which better explains the diversity is the geology (38.9\% of the variance), associated to axis 1 . As regards the other variables, altitude explains $28.4 \%$ of the variabil-

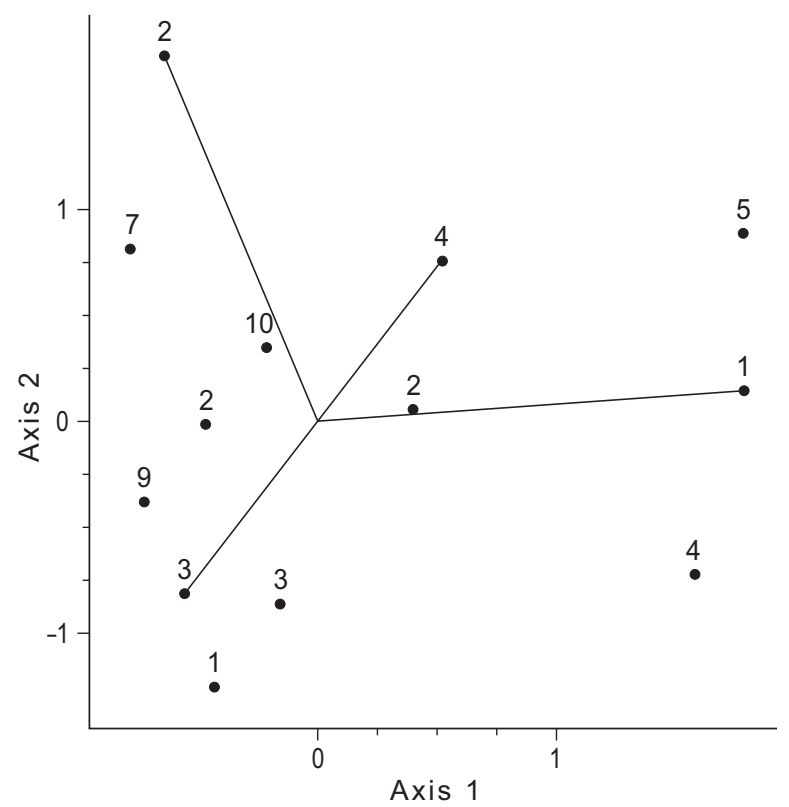

Figure 4: Biplot of environmental features versus considered syntaxa.

Slika 4: Dvorazsežnostni diagram rastiščnih dejavnikov in obravnavanih sintaksonov.

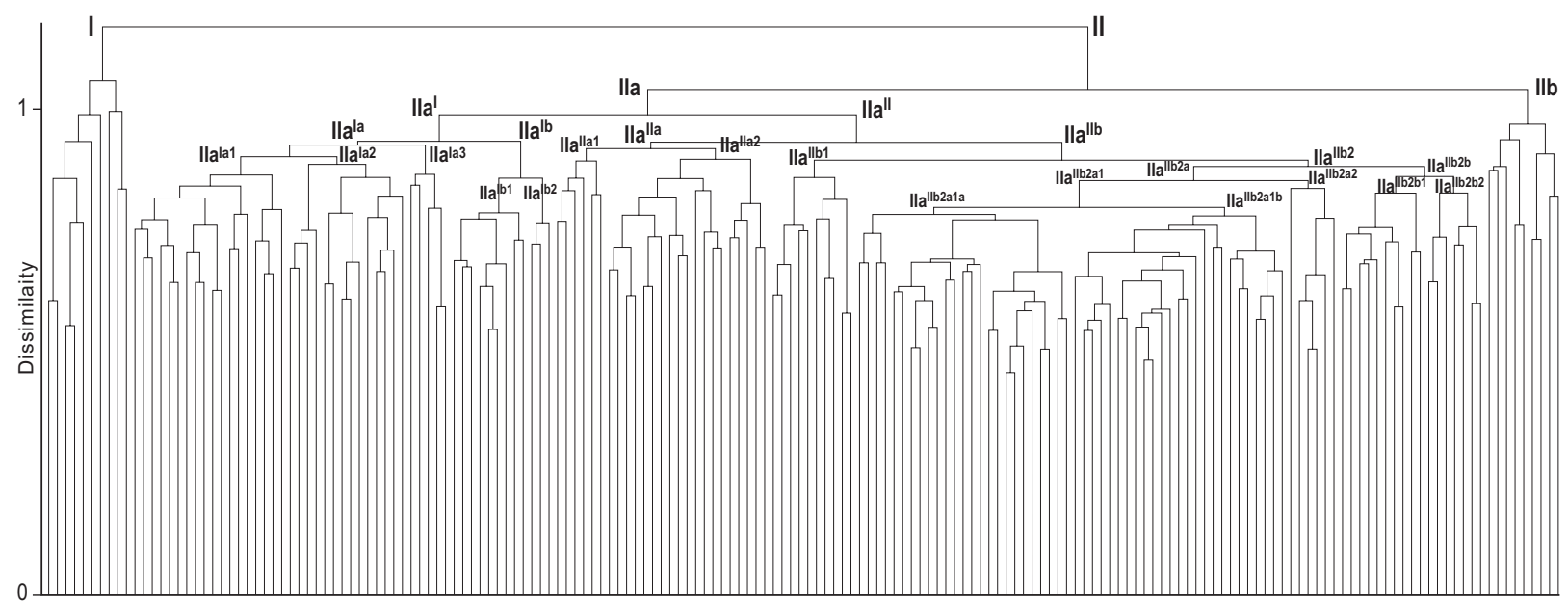

Figure 3: Cluster analysis of the studied forest plant communities. Slika 3: Klastrska analiza proučevanih gozdnih rastlinskih združb. 
ity and is clearly linked to axis 2 , while morphology, which explains $21.1 \%$ is linked to axis 3 .

Weighted phytosociological spectra highlight that cluster I, ascribed to Quercetalia pubescentipetraeae order, has a Quercetalia pubescenti-petraeae weighted cover value of $45-50 \%$ and Fagetalia sylvaticae cover value of 30-35\%. Relevés of cluster II, instead, highlight the change of the dominant phytosociological group, which becomes Fagetalia sylvaticae order. More in particular, sub-cluster IIa shows values of Quercetalia pubescenti-petraeae elements spanning from $7-10 \%$ to $16-18 \%$, while Fagetalia sylvaticae ones range from $65 \%$ to $79 \%$; sub-cluster IIb has Quercetalia pubescenti-petraeae values of $1-5 \%$, while those of Fagetalia sylvaticae are between $81 \%$ and $90 \%$.

\subsubsection{Beech and hop hornbeam mixed woods}

Beech and hop hornbeam mixed woods grow mainly on fairly steep North-facing slopes, ranging from $700-750$ to $950-1050 \mathrm{~m}$ a.s.l. They represent the phytosociological and ecological elements identifying the transition from Laburno anagyroidis-Ostryenion carpinifoliae (Carpinion orientalis, Quercetalia pubescenti-petraeae) hilly woods to mountain ones referred to Fagetalia sylvaticae order. As supposed by Catorci et al. (2003) a number of historical considerations (Reali 1871, Salbitano 1989, Catorci 2007) allow one to hypothesize the past existence of a beech wood type covering North-facing slopes between 600 and $900 \mathrm{~m}$ a.s.l. of which actual Ostrya carpinifolia and Fagus sylvatica subsp. sylvatica mixed woodlands could represent the xeric and degraded look, because of human activities and the consequent soil erosion. Such primeval formations could have been mixed woodlands, where Tilia plathyphyllos subsp. plathyphyllos, Acer sp. pl., Fagus sylvatica subsp. sylvatica, Carpinus betulus, Quercus cerris, Ilex aquifolium and, perhaps, Abies alba had a dominant role and where secular forestry and pasture activities allowed Ostrya carpinifolia settlement.

\section{Carici digitatae-Ostryetum carpinifoliae Catorci, Gatti et Sparvoli 2003}

(Group I, Table 3, relevés 1-7; holotypus rel. 20 of Table 2 in Catorci et al. 2003)

Woodland with a dominance of Ostrya carpinifolia and Fagus sylvatica subsp. sylvatica, widespread on North-facing slopes (from NW to E), with slope angle ranging from $10^{\circ}$ to $55^{\circ}$, at alti- tudes from 700 to $900 \mathrm{~m}$ (upper mesotemperate biclimatic belt).

This syntaxon is characterized by soils with the following features: $50-100 \mathrm{~cm}$ depth; $6.2-6.8 \mathrm{pH}$, tendly clayey texture (35-45\% sand, 20-30\% silt, $25-35 \%$ clay).

From a phytosociological point of view, these woods are characterized by the dominance of Quercetalia pubescenti-petraeae elements (45-50 \%) associated to a group of Fagetalia sylvaticae species (30-35\%).

Floristic analysis enabled classification of such a plant community into Carici digitatae-Ostryetum carpinifoliae association, described for the first time by Catorci et al. (2003) in low-mountain woodlands of Macerata Apennines (Central Italy) and mentioned also by Taffetani et al. (2004), who downgrades the association to the subassociation rank (Lathyro veneti-Fagetum sylvaticae staphyletosum pinnatae). However, on the basis of the historical (Catorci et al. 2003), physiognomical (hop hornbeam-beech woods versus beech woods) and phytosociological characterization (first of all the dominance of Quercetalia pubescenti-petraeae elements with respect to those belonging to Fagetalia sylvaticae), this placement is considered inappropriate, so the association level is mantained.

Moreover, Catorci et al. (2003) placed these syntaxa in Geranio versicoloris-Fagion sylvaticae, but the phytosociological framework and the clear division emerging from cluster analysis led to the placement of Carici sylvaticae-Ostryetum carpinifoliae in Laburno anagyroidis-Ostryenion carpinifoliae (Carpinion orientalis).

\subsubsection{Beech woods}

Beech woods are divided into two main groups referred to different altitudinal intervals (1000$1400 \mathrm{~m}$ and $1400-1800 \mathrm{~m}$ ) and consequently to different bioclimatic belts (lower and upper supratemperate). As better described later on, they are respectively referred to Lathyro veneti-Fagetum sylvaticae and Cardamino kitaibelii-Fagetum sylvaticae associations, described or confirmed by $\mathrm{Bi}$ ondi et al. (2002) on the basis of phytosociological tables including beech woods growing below $1300-1350 \mathrm{~m}$ a.s.l. only and which, therefore, could not represent the whole floristic diversity of Central Apennines beech woods (timberline is placed at about $1800-1850 \mathrm{~m}$ a.s.l.). Moreover, a discrepancy was observed between the species 
indicated by the authors as characteristic or differential (considered in this work) and those reported in the respective tables.

For such reasons it is considered appropriate to perform a screening and review of such species groups. In particular, Biondi et al. (2002) suggested as characteristic species of Lathyro veneti-Fagetum sylvaticae association: Cyclamen hederifolium subsp. hederifolium, Lathyrus venetus, Sorbus aria subsp. aria, Galanthus nivalis, Scilla bifolia, Corydalis cava subsp. cava and Viola alba subsp. dehnhardtii. In the present study Sorbus aria subsp. aria, Corydalis cava subsp. cava, Galanthus nivalis and Scilla bifolia are not confirmed, because the investigation highlighted that they are widespread between 600 and $1800 \mathrm{~m}$ a.s.l. (Table 4), thus they can not be considered as characteristic or differential species of Lathyro veneti-Fagetum sylvaticae association (low-hilly beech woods). Furthermore, the above mentioned species groups are integrated with $P o$ lygonatum multiflorum, Anemone apennina subsp. apennina and Anemone nemorosa, high-hilly and low-mountain geophytes which are found in the study area in the range of $800-1400 \mathrm{~m}$ a.s.l. In regard to Cardamino kitaibelii-Fagetum sylvaticae, Biondi et al. (2002), in accordance with Ubaldi (1993), list the following characteristic species: Cardamine kitaibelii, C. enneaphyllos, Anemone nemorosa, Epilobium montanum, Polystichum aculeatum, Adoxa moschatellina subsp. moschatellina and Taxus baccata. Anemone nemorosa and Taxus baccata are not confirmed as characteristic species in the present study because they proved to be widespread in the lower supratemperate bioclimatic belt and only sporadically in the upper supratemperate bioclimatic belt. Instead, Cardamine kitaibelii, C. enneaphyllos, Polystichum aculeatum, Epilobium montanum and Adoxa moschatellina subsp. moschatellina are confirmed, with the addition of Lathyrus vernus subsp. vernus.

Lathyro veneti-Fagetum sylvaticae Biondi, Casavecchia, Pinzi, Allegrezza et Baldoni 2002 lathyretosum veneti Biondi, Casavecchia, Pinzi, Allegrezza et Baldoni 2002

(Group IIa ${ }^{\text {Ia22 }}$, Table 5, relevés 19-32; holotypus rel. 3 of Table 2 in Biondi et al. 2002)

Woodland with a dominance of Fagus sylvatica subsp. sylvatica and Acer opalus subsp. obtusatum, that are managed as coppice with standards, widespread on mainly North-facing slopes with an inclination of $5-45^{\circ}$ and at altitudes ranging from (750) 900 to 1300 (1400) m a.s.l.
This syntaxon is characterized by soils with the following features: $100-150 \mathrm{~cm}$ depth; 6.0-7.0 $\mathrm{pH}$, clayey/tendly clayey texture (25-35\% sand, 25-35. silt, 40-50 clay).

From a phytosociological point of view these woodlands are characterized by the dominance of Fagetalia sylvaticae elements (65-70 \%) associated to a remarkable Quercetalia pubescenti-petraeae species group (15-20\%).

Floristic analysis allowed such a plant community to be placed in the Lathyro veneti-Fagetum sylvaticae lathyretosum veneti subassociation described for the first time by Biondi et al. (2002) for the Central Apennines low-mountain and signaled by other authors (Allegrezza 2003, Catorci et al. 2003, Biondi et al. 2004, Taffetani et al. 2004) for the Umbria-Marches Apennines (Central Italy).

Floristic comparison between the tables of Lathyro veneti-Fagetum sylvaticae and Carici digitatae-Ostryetum carpinifoliae highlights that the two associations are not well distinguished if only characteristic species are considered (characteristic species of Lathyro veneti-Fagetum sylvaticae well separate these beech woods from the high-mountain ones, but they are substantially present in Carici digitatae-Ostryetum carpinifoliae). This lack of differentiation seems to be further evidence that beech-hop hornbeam woods originate from degradation (alteration of structure and composition of tree and shrubs layers) of low-mountain beech woodlands. From a floristic point of view, the differentiation between the two syntaxa is due to the different weight of Quercetalia pubescentipetraeae species group.

More detailed analysis of groups of relevés forming sub-cluster IIa $^{\text {Ia }}$ highlighted the lower rank phytosociological units, described as follows.

Lathyro veneti-Fagetum sylvaticae Biondi, Casavecchia, Pinzi, Allegrezza et Baldoni 2002 lathyretosum veneti Biondi, Casavecchia, Pinzi, Allegrezza et Baldoni 2002 galium aparine variant

(Group IIa ${ }^{\mathrm{Ia} 3}$, Table 5, relevés 33-37)

Relevés 33-37 of Table 5 differ from those of typical subassociation for the presence of open site and ruderal type species. These beech communities grows on slopes with outcropping rock, often in morphological conditions of impluvium or in areas recently managed as high-forest, with high Nitrogen content. This peculiar ecological 
connotation is well emphasized by the variant of differential species block: Galium aparine (expression of high organic matter content and disturbance of soil), Digitalis lutea subsp. australis, Ribes alpinum (expression of high light availability within the forest community) and Doronicum columnae (expression of rocky surfaced substratum).

Lathyro veneti-Fagetum sylvaticae Biondi, Casavecchia, Pinzi, Allegrezza et Baldoni 2002 hieracietosum murorum (Allegrezza 2003) stat. nov. (=hieracio racemosi-fagetum sylvaticae luzuletosum sylvaticae Allegrezza 2003)

(Group IIa ${ }^{\mathrm{Ia} 1}$, Table 5, relevés 1-18; typus rel. 2 Table 37 in Allegrezza 2003)

Relevés 1-18 of Table 5 differentiate, as regards the ones ascribed to the typical subassociation, for most constant occurrence and higher cover values of some acidophilous species (Hieracium murorum, H. racemosum, Potentilla micrantha, Rosa arvensis, Cephalanthera rubra, Luzula forsteri, L. sylvatica subsp. sylvatica, Hypericum androsaemum, Carex sylvatica subsp. sylvatica and, in the tree layer, Quercus cerris or, locally, Castanea sativa).

Local characteristics of such phytocoenosis are similar to those of the typical subassociation, except for the soils developed on chert-rich bedrocks, such as the Calcari Diasprini Formation (AA.vv. 1991), or the deeper, partially decalcified and clay enriched colluvial soils accumulated on gentler slopes, partially decalcified and/or subjected to clay illuviation.

This syntaxon is characterized by soils with the following features: $100-150 \mathrm{~cm}$ depth, 5.8-6.2 $\mathrm{pH}$, clayey texture (20-30\% sand, 25-35. silt, 45-55 clay).

The above said floristic-ecological features led to the definition of a new subassociation named Lathyro veneti-Fagetum sylvaticae hieracietosum murorum, of which Rosa arvensis, Carex digitata, $C$. sylvatica subsp. sylvatica, Hieracium murorum, Potentilla micrantha, Luzula forsteri and Platanthera chlorantha are differential species.

It must be clarified that Hieracio racemosiFagetum sylvaticae luzuletosum sylvaticae, described by Allegrezza (2003) for Monte San Vicino (Marches Region), that develops on the same substratum type, in this work is downgraded to the subassociation rank (Lathyro veneti-Fagetum sylvaticae hieracietosum murorum). Indeed, as pointed out in the synoptic table (Table 5), characteristic and differential species of the typical subassociation still occur and, moreover, there are no remark- able differences regarding acidophilous species between the new proposed subassociation and Hieracio racemosi-Fagetum sylvaticae luzuletosum sylvaticae, while the main differences are due to the lower floristic richness of Allegrezza (2003) relevés. Characteristic species of Hieracio racemosi-Fagetum sylvaticae luzuletosum sylvaticae are used as differentials of subassociation in Lathyro veneti-Fagetum sylvaticae hieracietosum murorum, except for Digitalis lutea subsp. australis and Pteridium aquilinum subsp. aquilinum because they are marginal species, and for Lactuca muralis, Cephalanthera longifolia and Ilex aquifolium because widespread with the same frequency also in the typical subassociation.

Solidagini-Fagetum sylvaticae (Longhitano et Ronsisvalle 1974) Ubaldi, Zanotti, Puppi, Sparanza et Corbetta 1987 ex Ubaldi 1993 luzuletosum sylvaticae Catorci, Ballelli, Iocchi, Paura et Vitanzi 2008

(Group $\mathrm{IIa}^{\mathrm{Ib} 1}$, Table 7, relevés 1-9; holotypus rel. 15 of Table 11 in Catorci et al. 2008)

Woodland with a dominance of Fagus sylvatica subsp. sylvatica, managed as coppice with standards or high-forest, growing on sandstone Northfacing slopes (from $\mathrm{E}$ to WNW) with an inclination of $10-45^{\circ}$, at altitudes ranging from 800 to $1200 \mathrm{~m}$.

This syntaxon is characterized by soils with the following features: $100-150 \mathrm{~cm}$ depth, $5.0-6.0$ $\mathrm{pH}$, tendly silty/tendly clayey texture (50-60\% sand, 30-40 silt, 10-20 clay).

From a phytosociological point of view these woodlands are characterized by the dominance of Fagetalia sylvaticae elements (73\%) associated to a small group of species belonging to Quercetalia pubescenti-petraeae order (9\%); acidophilous species are also well represented (13-15\%).

Catorci et al. (2008) placed such woodlands into Solidagini-Fagetum sylvaticae (described by Ubaldi et al. 1987, Ubaldi 1995), but Biondi et al. (2008) observed that the association is not well described because the typical subassociation represents a strongly impoverished aspect of acidophilous beech woods. Such an observation led Biondi et al. (2008) to describe the new association Potentillo micranthae-Fagetum sylvaticae, widespread on the Laga Mountains arenaceous Flysch, in the lower supratemperate bioclimatic belt.

Floristic analysis of the relevés of Table 7 highlights that the species suggested as characteristic 
or differential of Potentillo-Fagetum are absent or only sporadically present. For such reason it should be appropriate to mantain the placement of Monti Sibillini beech forests growing on sandstone substrata in Solidagini-Fagetum and, in particular, in Solidagini-Fagetum sylvaticae luzuletosum sylvaticae subassociation, described for the first time by Catorci et al. 2008.

A more detailed analysis of relevés groups composing cluster $\mathrm{IIa}^{\mathrm{Ia}}$ enables one to highlight a phytosociological lower rank unit, described as follows.

Solidagini-Fagetum sylvaticae (Longhitano et Ronsisvalle 1974) Ubaldi, Zanotti, Puppi, Sparanza et Corbetta 1987 ex Ubaldi 1993 aceretosum pseudoplatani Catorci, Ballelli, Iocchi, Paura et Vitanzi 2008

(Group IIa ${ }^{\mathrm{Ib} 2}$, Table 7, relevés 10-12; holotypus rel. 21 of Table 11 in Catorci et al. 2008)

Relevés 10-12 of Table 7 differentiate from the other part of the table because of the occurrence of Tilio-Acerion alliance species (Saxifraga rotundifolia subsp. rotundifolia, Fraxinus excelsior subsp. excelsior, Acer pseudoplatanus, Tilia platyphyllos subsp. platyphyllos, Acer platanoides, Polystichum setiferum) which identify Solidagini-Fagetum sylvaticae aceretosum pseudoplatani subassociation. As described by Catorci et al. (2008) such subassociation develops on very steep slopes $\left(45-70^{\circ}\right)$, in impluvia with a great amount of outcropping rocks and represents the catenal contact between Solidagini-Fagetum and Ornithogalo sphaerocarpiAceretum pseudoplatani (Tilio-Acerion alliance) described for the Monte dell'Ascensione gorges (Southern Marches) by Taffetani (2000).

Cardamino kitaibelii-Fagetum sylvaticae (Ballelli et Biondi 1982) Ubaldi, Zanotti, Puppi, Speranza et Corbetta 1987 ex Ubaldi 1993 cardaminetosum kitaibelii subass. nova

(Group IIa ${ }^{\mathrm{Ilb2a1b}}$, Table 8, relevés $1-25$; typus rel. 7 of Table 8 )

Woodland with a dominance of Fagus sylvatica subsp. sylvatica, mainly managed as high-forest, widespread on mainly North-facing slopes (from WNW to E) with an inclination of $15-60^{\circ}$, at altitudes ranging from (1200) 1400 to 1650 (1700) m (upper supratemperate bioclimatic belt).

This syntaxon is characterized by soils with the following features: $50-100 \mathrm{~cm}$ depth, 5.5-6.5 $\mathrm{pH}$, tendly clayey texture (35-45\% sand, 25-35 silt, 25-35 clay).
From a phytosociological point of view these woodlands are characterized by the dominance of Fagetalia sylvaticae order elements (80-85 \%) associated to a small group of species belonging to Quercetalia pubescenti-petraeae order (4-5\%).

Floristic analysis consented to the placement of such vegetation into Cardamino kitaibelii-Fagetum sylvaticae association, described for the first time by Ubaldi et al. (1987) for the Northern Marches Apennines and signaled by other authors (Biondi et al. 2002, 2004, 2008, Di Pietro 2002, Catorci et al. 2003, Pirone et al. 2003, 2005, Ciaschetti et al. 2006) for the Central Apennines sector of the Italian Peninsula.

According to the International Phytosociological Nomenclature Code (Weber et al. 2002), in the present work the typical subassociation is proposed with the cardaminetosum kitaibelii epithet (typus rel. 7 of Table 8).

A more detailed analysis of relevé groups forming cluster $\mathrm{IIa}^{\mathrm{Ia}}$ allowed one to highlight some phytosociological lower rank units, described in the following paragraph.

Cardamino kitaibelii-Fagetum sylvaticae (Ballelli et Biondi 1982) Ubaldi, Zanotti, Puppi, Speranza et Corbetta 1987 ex Ubaldi 1993 cardaminetosum kitaibelii subass. nova sorbus aucuparia variant

Relevés $26-35$ of Table 8 (Group IIa ${ }^{\mathrm{Ib} 2 \mathrm{~b} 1}$ ) are characterized by the occurrence of a high number of species belonging to Fagetalia sylvaticae order (82\%) and a smaller number of elements referred to Quercetalia pubescenti-petraeae order (7\%). Thus, this is a variant of Cardamino kitaibeliiFagetum sylvaticae cardaminetosum kitaibelii, which develops on convex morphologies (broad ridge) in areas with outcropping rock and shallow soil. Such variant is characterized by Sorbus aucuparia subsp. aucuparia, Sorbus aria subsp. aria and Laburnum alpinum, and describes a semi-open forest situation.

Cardamino kitaibelii-Fagetum sylvaticae (Ballelli et Biondi 1982) Ubaldi, Zanotti, Puppi, Speranza et Corbetta 1987 ex Ubaldi 1993 cardaminetosum kitaibelii subass. nova polystichum setiferum variant

Relevés 36-42 of Table 8 (Group $\mathrm{IIa}^{\mathrm{IIb} 2 \mathrm{~b} 2}$ ) are characterized by the presence of a high percentage of Fagetalia sylvaticae order species (79 \%) and a small group of Quercetalia pubescenti-petraeae order (8\%). Therefore, it represents a variant of 
Cardamino kitaibelii-Fagetum sylvaticae cardaminetosum kitaibelii, developing in "impluvia", where outcropping rock and deep soil pockets, often covered by detritic matter, are alternated.

Such variant is characterized by Polystichum setiferum and Asperula taurina subsp. taurina; in the tree layer Acer pseudoplatanus, A. platanoides and Fraxinus excelsior subsp. excelsior occur, showing a possible transition towards gorge formations referred to Tilio-Acerion.

Cardamino kitaibelii-Fagetum sylvaticae (Ballelli et Biondi 1982) Ubaldi, Zanotti, Puppi, Speranza et Corbetta 1987 ex Ubaldi 1993 anemonetosum nemorosae subass. nova

(Group IIa ${ }^{\mathrm{II} 2}$, Table 9, relevés 7-25; typus rel. 16 of Table 9 )

Relevés of Table 9 differ from those of the typical subassociation by the relatively high number of elements belonging to Quercetalia pubescenti-petraeae (about $10 \%$ ), a rate that in Lathyro veneti-Fagetum sylvaticae is about $15-20 \%$, while in Cardamino kitaibelii-Fagetum sylvaticae cardaminetosum kitaibelii it is around 4-5\%. Stational characteristics of such subassociation are similar to those of typical subassociation, except for altitudinal range. In fact, the thermophilous aspect of this association is connected to an altitudinal interval ranging between (1100) 1200 and $1400 \mathrm{~m}$.

Floristic composition and altitudinal range allow one to define a new subassociation named Cardamino kitaibelii-Fagetum sylvaticae anemonetosum nemorosae, of which Anemone nemorosa, Epipactis leptochila, Hepatica nobilis, Acer opalus subsp. obtusatum and Luzula sylvatica subsp. sylvatica represent the differential species. This subassociation defines catenal contact between Lathyro veneti-Fagetum sylvaticae beech woods and those of Cardamino kitaibelii-Fagetum sylvaticae. Being a catenal contact, connected to an altitudinal gradient, transition between these syntaxa, is generally shaded and its significance can be better undertstood if, together with differential species, also the weight of Quercetalia pubescenti-petraeae elements and serial contacts are considered, as mentioned later on.

This syntaxon is characterized by soils with the following features: $50-100 \mathrm{~cm}$ depth, 5.5-6.5 $\mathrm{pH}$, tendly clayey texture (35-45\% sand, 25-35 silt, 25-35 clay).

Inside this subassociation, a facies at Orthilia seconda is identified, which differs also for the oc- currence of Luzula forsteri and Polypodium interjectum.

Cardamino kitaibelii-Fagetum sylvaticae Ubaldi 1993 ex Ubaldi, Zanotti, Puppi, Speranza et Corbetta 1987 anemonetosum nemorosae subass. nova festuca heterophylla variant

Relevés 1-6 of Table 9 differ from Cardamino kitaibelii-Fagetum sylvaticae anemonetosum nemorosae for the presence of some sub-acidophilous species, which identify a new variant named Cardamino kitaibelii-Fagetum sylvaticae anemonetosum nemorosae Festuca heterophylla variant, characterized by: Festuca heterophylla and Hieracium murorum. This variant develops on sub-acid soils, probably due to the accumulation of organic matter.

Cardamino kitaibelii-Fagetum sylvaticae Ubaldi 1993 ex Ubaldi, Zanotti, Puppi, Speranza et Corbetta 1987 corallorhizetosum trifidae subass. nova

(Group IIa ${ }^{\mathrm{Ib} 2 a 1 a}$, Table 10, relevés 1-25; holotypus rel. 8 of Table 10)

Relevés of Table 10 are different from those of typical subassociation for the occurrence of some high-altitude beech woods species, which identify a new subassociation, named Cardamino kitaibelii-Fagetum sylvaticae corallorhizetosum trifidae, of which Actaea spicata, Corallorhiza trifida, Polystichum lonchitis and Silene dioica are proposed as differential species. In such subassociation, species belonging to Quercetalia pubescenti-petraeae order are nearly absent, favouring the ones of Fagetalia sylvaticae order, which exceed $90 \%$. This subassociation mainly develops on slopes, in an altitudinal belt close to the upper ecological timberline, from (1600-1800 m a.s.l.). Such woodlands are structurally managed as high-forest, often with signs of geotropism.

This syntaxon is characterized by soils with the following features: $50-100 \mathrm{~cm}$ depth, 5.5-6.5 $\mathrm{pH}$, tendly clayey texture (35-45\% sand, 25-35 silt, 25-35 clay).

Cardamino kitaibelii-Fagetum sylvaticae Ubaldi 1993 ex Ubaldi, Zanotti, Puppi, Speranza et Corbetta 1987 corallhorizetosum trifidae subass. nova moehringia trinervia variant

Relevés 26-31 of Table 10 (Group $\mathrm{IIa}^{\mathrm{IIb} 2 \mathrm{a} 2}$ ) differ from the ones ascribed to Cardamino kitaibelii-Fagetum sylvaticae corallhorizetosum trifidae for the presence of some nitrophilous and helio- 
philous species, which identify a new variant, named Cardamino kitaibelii-Fagetum sylvaticae corallhorizetosum trifidae Moehringia trinervia variant, characterized by Moehringia trinervia, Lamium maculatum, Myosotis nemorosa, Aegopodium podagraria, Campanula micrantha and La-mium garganicum subsp. laevigatum. This variant develops in correspondence with the most exter-nal ramifications of woods as well as on its marginal areas, or in situations characterized by high brightness in the undergrowth and high Nitrogen content in the soil (probably because cattle use these areas as a rest place during the hottest hours of the day).

\section{Impoverished facies of Cardamino kitaibelii- Fagetum sylvaticae}

(Group IIa ${ }^{\mathrm{IIb} 1}$, Table 11, relevés 1-10)

Relevés of Table 11 are characterized by a general decrease of floristic richness due to two different situations which originate the same phenomenon in terms of floristic composition. In the first case, there are occurrences of quite high values of Laburnum anagyroides subsp. anagyroides and Brachypodium rupestre, indicators of openings in the tree layer and/or of an early stage of the forest population. In the second case, species impoverishment is due to the accumulation of thick litter $(10-15 \mathrm{~cm})$ on the soil, which prevents the development of the smallest nemoral species. It must be noticed that such situations are frequently associated with woods that have not been cut for many decades, but that can not be considered old-growth forest yet.

Aceretum obtusati-Pseudoplatani Biondi, Casavecchia, Pinzi, Allegrezza et Baldoni 2002

(Group IIb, Table 12, relevés 1-9; holotypus rel. 1 of Table 6 in Biondi et al. 2002)

Woodland with Acer pseudoplatanus, Fraxinus excelsior subsp. excelsior and Tilia platyphyllos subsp. platyphyllos dominance, generally unmanaged, growing in deep valleys and narrow and steep impluvia, with high levels of edaphic and atmospheric moisture, with slope angles of $20-55^{\circ}$, at altitudes ranging from 1000 to $1400 \mathrm{~m}$.

From a phytosociological point of view these woodlands are characterized by the dominance of Fagetalia sylvaticae order and Tilio-Acerion alliance species.

Floristic analysis allowed such phytocoenosis to be placed into Aceri obtusati-pseudoplatani association, described for the first time by Biondi et al. (2002) for the Central and Northern Apennines woodlands and signaled by other authors (Paura \& Cutini 2006, Catorci et al. 2008) for the Central-Southern Apennines sector of the Italian Peninsula.

\section{DISCUSSION}

The results of phytosociological analysis emphasize that the floristic and phytocoenotic diversity of beech woods is substantially related to an altitudinal gradient and, secondarily, to geo-pedological aspects (Figure 5).

In particular, it was possible to highlight a clear trend in the ratio between the number of elements of Quercetalia pubescenti-petraeae and Fagetalia sylvaticae orders, as previously partly discussed, which can be a good support system for phytosociological diagnosis.

Indeed, syntaxa with Ostrya carpinifolia dominance (Scutellario columnae-Ostryetum carpinifoliae Pedrotti, Ballelli \& Biondi 1982 ex Pedrotti et al. 1980, Aceri obtusati-Quercetum cerridis, etc.), belonging to the upper mesotemperate belt, show a group of Quercetalia pubescenti-petraeae species higher than 70-75\% (Ballelli et al. 1982, Ubaldi \& Speranza 1985, Catorci \& Orsomando 2001). This percentage decreases around $45-50 \%$ for Carici digitatae-Ostryetum carpinifoliae, which identifies a transition from Quercetalia pubescenti-petraeae to Fagetalia sylvaticae. Inside this last order, Lathyro veneti-Fagetum sylvaticae is characterized by the occurrence of Quercetalia pubescenti-petraeae elements, never lower than $15 \%$ and never higher than $25 \%$, while Cardamino kitaibelii-Fagetum sylvaticae shows values of Quercetalia pubescenti-petraeae elements ranging from 3 to $5 \%$ as regards the typical subassociation, from 8 to $12 \%$ for Cardamino kitaibelii-Fagetum sylvaticae anemonetosum nemorosae subassociation (growing at lower altitudes) and lower than $3 \%$ for Cardamino kitaibelii-Fagetum sylvaticae corallorhizetosum trifidae (microthermal subassociation).

A further control and screening system of syntaxonomical aspects can derive from the analysis of mantle shrub vegetation that, on the basis of the previous set of knowledge (Catorci \& Orsomando 2001, Allegrezza 2003, Biondi et al. 2004, Catorci et al. 2007, 2008) and of the current studies, shows significant correlations with the considered forest types. Such correlations are shown in Table 13. 


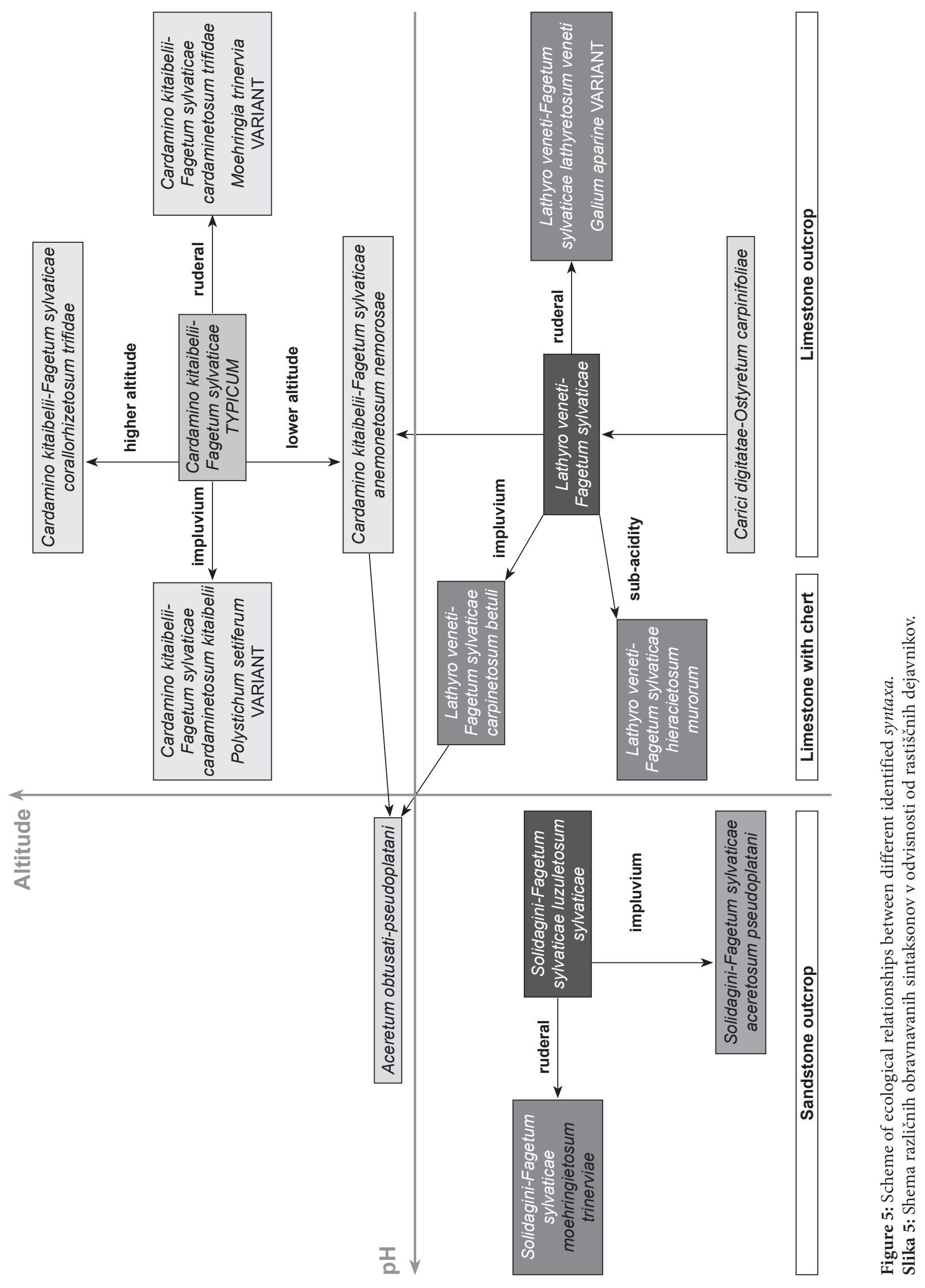




\section{CONCLUSIONS}

Phytosociological placement of Fagus sylvatica woods of Monti Sibillini National Park confirmed the syntaxonomical scheme as defined by Biondi et al. (2002), allowing, however, for a more complete understanding of Central Apennines beech woods from an ecological and diagnostic viewpoint, thanks to the wider altitudinal interval considered.

Moreover, this work emphasizes that forest structure, management types, felling frequency and other parameters related to the historical soil use assume a remarkable role in the floristic characterization of these woodlands, as also highlighted by Bartha et al. (2008). The continuation of the research in this direction can supply important indications for management, a fundamental aspect because the surveyed beech woods are included into a National Park and almost completely into Natura 2000 Network sites, according the Habitat Directive 92/43/CEE (in which investigated beech woods are part of priority Habitat "9210 - Apennines Taxus and Ilex beech woods").

On the whole, the beech forest ecosystem of the Sibillini massif is composed of about 250 species representing $13 \%$ of the Monti Sibillini National Park flora (Ballelli et al. 2010, in press). Among these species as deserving of attention in terms of rarity, threat and vulnerability, are Asarum europaeum, Convallaria majalis, Corallorhiza trifida, Euonymus verrucosus, Scutellaria altissima, etc.

Finally, we can say that the beech forest diversity is an important core for the Monti Sibillini biodiversity.

\section{Syntaxonomical scheme}

QUERCO-FAGETEA Br.-Bl. 1948 em. Ohba 1974

Quercetalia PUbescentis-Petraeae Klika 1933 Carpinion orientalis Horvat 1958

Laburno anagyroidis-Ostryenion carpinifoliae (Ubaldi 1995) Blasi, Di Pietro et Filesi 2004

Carici digitatae-Ostryetum carpinifoliae $\mathrm{Ca}-$ torci, Gatti et Sparvoli 2003

Fagetalia SYlvaticae Pawlowski in Pawlowski, Sokolowski et Wallish 1928

Geranio versicoloris-Fagion sylvaticae Gentile 1969

Lathyro veneti-Fagetum sylvaticae Biondi, Casavecchia, Pinzi, Allegrezza et Baldoni 2002 lathyretosum veneti Biondi, Casavecchia, Pinzi, Allegrezza et Baldoni 2002

Galium aparine variant

hieracietosum murori (Allegrezza 2003)

Catorci, Vitanzi et Ballelli stat. nov. Aremonio-Fagion sylvaticae (Horvat 1938) Torok, Podani et Borhidi1989

Solidagini-Fagetum sylvaticae (Longhitano et Ronsisvalle 1974) Ubaldi, Zanotti, Puppi, Sparanza et Corbetta 1987 ex Ubaldi 1993 luzuletosum sylvaticae Catorci, Ballelli, Iocchi, Paura et Vitanzi 2008 aceretosum pseudoplatani Catorci, Ballelli, Iocchi, Paura et Vitanzi 2008

Cardaminokitaibelii-Fagetumsylvaticae Ubaldi, Zanotti, Puppi, Speranza et Corbetta 1987 ex Ubaldi 1993 cardaminetosum kitaibelii subass. nova Sorbus aucuparia variant Polystichum setiferum variant anemonetosum nemorosae subass. nova Festuca heterophylla variant corallorhizetosum trifidae subass. nova Moehringia trinervia variant

Tilio platyphylli-Acerion pseudoplatani Klika 1955

Ostryo carpinifoliae-Tilienion plathyphylli Košir, Čarni et Di Pietro 2008

Aceretum obtusati-pseudoplatani Biondi, Casavecchia, Pinzi, Allegrezza et Baldoni 2002

\section{REFERENCES}

AA.vv. 1991: L'ambiente fisico delle Marche. Geologia Geomorfologia Idrogeologia. Giunta Regionale - Assessorato Urbanistica e Ambiente, Ancona, 255 pp.

Allegrezza, M. 2003: Vegetazione e paesaggio vegetale della dorsale del Monte San Vicino (Appennino centrale). Fitosociologia 40 (1) suppl. 1: 3-118.

Allegrezza, M. \& Biondi, E. 2008: Studio fitosociologico dell'area forestale degli "Abeti soprani" (Alto Molise - Appennino meridionale). Fitosociologia 45 (1): 161-176.

Allegrezza, M., Baldoni, M.A., Biondi, E., Taffetani, F. \& Zuccarello, V. 2002: Studio fitosociologico dei boschi a Quercus pubescens s.l. delle Marche e delle zone contigue dell'Appennino centro-settentrionale (Italia centrale). Fitosociologia 39 (1): 161-172. 
A.S.S.A.M. 2006: Suoli e paesaggi delle Marche. Regione Marche, Ancona, 303 pp.

Ballelli, S., Biondi, E. \& Pedrotti, F. (1982): L'associazione Scutellario-Ostryetum dell'Appennino centrale. In: Pedrotti, F. (ed.), Guide-Itinéraire. Excursion Internationale de Phytosociologie en Italie centrale (2-11 juillet 1982). Univ. Camerino: 565-569.

Ballelli, S., Gatti, R., Raponi, M. \& Catorci, A. 2006: Aspetti vegetazionali e floristici del territorio nursino (Umbria - Italia centrale): le serie di vegetazione della roverella (Quercus pubescens s.l.). Webbia 61 (2): 305-323.

Ballelli, S., Catorci, A., Cesaretti, S., Gatti, R., Montenegro, B. \& Vitanzi, A. 2010: La flora bibliografica dei Monti Sibillini (Appennino centrale - Italia). Braun-Blanquetia 47 (in press).

Bartha, S., Campetella, G., Ruprecht, E., Kun, A., Házi, J., Horváth, A., Virágh, K. \& Molnár, Z. 2008: Will interannual variability in sand grassland communities increase with climate change? Community Ecology 9: 13-21.

Biondi, E. 1996: L'analisi fitosociologica nello studio integrato del paesaggio. In: Loidi, J. (ed.) Avances en Fitosociología. Universidad del Pais Vasco, pp. 13-22.

Biondi, E., Baldoni, M.A. \& Talamonti, M.C. 1995: Il fitoclima delle Marche. In: Atti del Convegno "Salvaguardia e gestione dei beni ambientali nelle Marche" (Ancona, 8-9 aprile 1991). Tipolit. Trifogli, Ancona, pp. 21-70.

Biondi, E., Pinzi, M. \& Gubellini, L. 2004: Vegetazione e paesaggio vegetale del Massiccio del Monte Cucco (Appennino centrale, Dorsale Umbro-Marchigiana). Fitosociologia 41 (2) suppl. 1: 3-81.

Biondi, E., Casavecchia, S., Pinzi, M., Allegrezza, M. \& Baldoni, M.A. 2002: The syntaxonomy of the mesophilous woods of the Central and Northern Apennines (Italy). Fitosociologia 39 (2): 71-93.

Biondi, E., Casavecchia, S., Frattaroli, A.R., Pirone, G., Pesaresi, S., Di Martino, L., Galassi, S., Paradisi, L., Ventrone, F., Angelini, E. \& Ciaschetti, G. 2008: Forest vegetation of the Upper Valley of the Vomano Valley (central Italy). Fitosociologia 45 (1): 117-160.

Blasi, C., Di Pietro, R. \& Filesi, L. 2004: Syntaxonomical revision of Quercetalia pubescenti-petraeae in the Italian Peninsula. Fitosociologia 41 (1): 87-164.

Blasi, C., Fortini, P., Grossi, G. \& Presti, G. 2005:
Faggete e cerrete mesofite dell'Alto Molise. Fitosociologia 42 (2): 67-81.

Bonin, G. 1967-69: A propos des forêts de Hêtre dans le massif du Pollino (Calabre). Annali di Botanica 29: 157-165.

Braun-Blanquet, J. 1931: Pflanzensoziologie. Grundzüge der vegetationnskunde. SpringerVerlag, Wien.

Braun-Blanquet, J. 1964: Pflanzensoziologie. $3^{\text {rd }}$ ed. Springer, Wien - New York.

Brullo, S. 1983: Contributo alla conoscenza della vegetazione delle Madonie (Sicilia settentrionale). Bollettino Accademia Gioenia di Scienze Naturali Catania 16 (322), 351-420.

Burrascano, S., Rosati, L. \& Blasi, C. 2009: Plant species diversity in Mediterranean old-growth forests: a case study from central Italy. Plant Biosystems 143 (1): 190-200. DOI 10.1080/112 63500802709699.

Calandra, R. \& Leccese, A. 2007: La carta dei suoli del territorio del Parco dei Monti Sibillini (con esempio di carta derivata). In: Biondi, E. (ed.), Atti del $43^{\circ}$ congresso della Società Italiana Scienza della Vegetazione, "L'applicazione della Direttiva Habitat in Italia e in Europa". Fitosociologia 44 (2) suppl. 1: 363-366.

Catorci, A. 2007: Lineamenti storico-economici del paesaggio pastorale maceratese. In: Catorci, A. \& Gatti, R. (eds.), Le praterie montane dell'Appennino maceratese. Braun-Blanquetia 42: 39-45.

Catorci, A. \& Orsomando, E. 2001: Note illustrative della Carta della Vegetazione del Foglio Nocera Umbra (N. 312 - Carta d'Italia I.G.M. - 1 : 50.000). Braun-Blanquetia 23: 1-129.

Catorci, A., Gatti, R. \& Sparvoli, D. 2003: Contributo alla conoscenza dei boschi basso montani dell'Appennino maceratese (Marche, Italia centrale). Fitosociologia 40 (2): 43-53.

Catorci, A., Cesaretti, S., Pancotto, D. \& Vitanzi, A. 2007: Analisi della vocazionalità del territorio della Comunità Montana di Camerino per la produzione di biomasse solide agro-forestali ad uso energetico. In: Catorci, A., Cesaretti, S. \& Marchetti, P. (eds.), Vocazionalità del territorio della Comunità Montana di Camerino per la produzione di biomasse solide agro-forestali ad uso-energetico. L'uomo e l'ambiente 47: 26-60.

Catorci, A., Vitanzi, A., Paura, B., Iocchi, M. \& Ballelli, S. 2008: La vegetazione forestale dei substrati arenacei della Val d'Aso (Marche, Italia centrale). Fitosociologia 45 (2): 41-76. 
Ciaschetti, G., Pirone, G., Frattaroli, A.R. \& Corbetta, F. 2006: La vegetazione del Piano di Pezza (Parco Naturale Regionale "Sirente-Velino" - Italia Centrale). Fitosociologia 43 (1): 67-84.

Conti, F., Abbate, G., Alessandrini, A. \& Blasi, C. 2005: An Annotated Checklist of the Italian Vascular Flora. Palombi Editore, Roma, 420 pp.

Conti, F., Alessandrini, A., Bacchetta, G., Banfi, E., Barberis, G., Bartolucci, F., Bernardo, L., Bonacquisti, S., Bouvet, D., Bovio, M., Brusa, G., Del Guacchio, E., Foggi, B., Frattini, S., Galasso, G., Gallo, L., Gangale, C., Gottschlich, G., Grünanger, P., Gubellini, L., Iriti, G., Lucarini, D., Marchetti, D., Moraldo, B., Peruzzi, L., Poldini, L., Prosser, F., Raffaelli, M., Santangelo, A., Scalssellati, E., Scortegagna, S., Selvi, F., Soldano, A., Tinti, D., Ubaldi, D., Uzunov, D. \& Vidali, M. (2007): Integrazioni alla checklist della flora vascolare italiana. Natura Vicentina 10 (2006): 5-74.

Credano, V., Ferrari, C., Pirola, A., Speranza, M. \& Ubaldi, D. 1980: Carta della vegetazione del crinale appenninico del Monte Giovo al Corno alle Scale. Collana Programma Finalizzato "Promozione Qualità Ambiente". Roma, C.N.R., AQ/1/81: 5-30.

Di Filippo, A., Piovesan, G. \& Schirone, B. 2004: Le foreste vetuste: criteri per l'identificazione e la gestione. XIV Congresso della Società Italiana di Ecologia. Siena.

Di Pietro, R. 2002: Fagus sylvatica woodlands in Southern Apennines. In: "Abstract of the international Symposium on Biodiversity \& Phytosociology". University of Ancona, September $18^{\text {th }}-19^{\text {th }}$ 2002: $13-14$.

Di Pietro, R. 2007: Coenological and syntaxonomical analysis of the beech woodlands of the Laga Mountains (central Italy). La biogeografia dell'Appennino centrale e settentrionale 30 anni dopo. Biogeographia 28: 45-118.

Di Pietro, R., Izco, J. \& Blasi, C. 2004: Contribution to the nomenclatural knowledge of Fagus sylvatica woodlands of Southern Italy. Plant Biosystems 138 (1): 27-52.

Franklin, J.F. \& Spies, T.A. 1991: Composition, function, and structure of old-growth Douglas-fir forests. In: Ruggiero, L.F., Aubry, K.B., Carey, A.B. \& Huff, M.H. (eds.), Wildlife and Vegetation of Unmanaged Douglas-fir Forests. USDA Forest Service General Technical Report PNW-GTR-285: 71-80.
Gehu, J.-M. \& Rivas-Martínez, S. 1981: Notions fondamentales de phytosociologie. Ber. Int. Simp. Int. Vereinigung Vegetationsk: 5-33.

Gentile, S. 1970: Sui faggeti dell'Italia meridionale. Atti Ist. Bot. Lab. Critt. Univ. Pavia, ser. 6 (5): 207-306.

Giovagnotti, C., Calandra, R., Leccese, A. \& Giovagnotti, E. 2003: I Paesaggi Pedologici e la Carta dei Suoli dell'Umbria. Camera di Commercio, Industria, Artigianato e Agricoltura di Perugia. Stampa Litografica srl, Todi.

I.P.L.A. 2001: I tipi forestali delle Marche. Regione Marche, Assessorato Agricoltura e Foreste. Ed. Diffusioni Grafiche s.p.a., Torino.

Košir, P., Čarni, A. \& Di Pietro, R. 2008: Classification and phytogeographical differentiation of broad-leaved ravine forests in southeastern Europe. Journal of Vegetation Science 19 (3): 331-342. DOI: 10.3170/2008-8-18372.

Orloci, L. 1978: Multivariate analysis in vegetation research. Junk, the Hague.

Paura, B. \& Cutini, M. 2006: Sull'ecologia delle foreste del Tilio-Acerion Klika 1955 in Molise e considerazioni sui caratteri cenologici e fitogeografici dei boschi di forra dell'Appennino centro-meridionale (Italia centrale e meridionale). Webbia 61 (1): 145-165.

Pedrotti, F. 1995: Il Parco Nazionale dei Monti Sibillini. Un parco tra leggenda e straordinarie emergenze naturali. Natura e Montagna. Anno XLII, 3/4.

Pignatti, S. 1982: Flora d'Italia. Edagricole, Bologna.

Pirone, G., Ciaschetti, G. \& Frattaroli, A.R. 2005: La vegetazione della Riserva Naturale Regionale "Abetina di Rosello" (Abruzzo, Italia). Fitosociologia 42 (1): 121-137.

Pirone, G., Ciaschetti, G., Frattaroli, A.R. \& Corbetta, F. 2003: La vegetazione della Riserva Naturale Regionale "Lago della Serenella" (Abruzzo-Italia). Fitosociologia 40 (2): 55-71.

Podani, J. 2001: Syntax 2000 computer program for data analysis in ecology and systematics. Budapest.

Poldini, L. \& Vidali, M. 1995: Prospetto sistematico della vegetazione nel Friuli-Venezia Giulia. Atti dei convegni Lincei, 115: 155-174.

Reali, A. 1871: Gli alberi e gli arbusti del circondario e dell'Appennino camerte. Camerino, Tip. Borgarelli.

Rivas-Martínez, S. 2004. Global Bioclimatics. http://www.globalbioclimatics.org (versione 2304-04, 27-08-04). 
Rivas-Martínez, S., Penas, A. \& Diaz, T.E. 2004: Biogeographic map of Europe. Cartographic Service, University of Léon, Spain.

Rivas-Martínez, S. 2005: Notions on dynamiccatenal phytosociology as a basis of landscape science. Plant Biosystem 139, (2): 135-144.

Rivas-Martínez, S. 2005a: Avances en Geobotanica. http://www.globalbioclimatics.org

Rosati, L., Di Pietro, R. \& Blasi, C. 2005: La vegetazione forestale della Regione Temperata del "Flysch del Cilento" (Italia meridionale). Fitosociologia 42 (2): 33-65.

Salbitano, F. 1989: Storia dei boschi del Monte Catria. In: Il bosco dell'Appennino. Centro Studi Valleremita. Comunità montana Alta Valle dell'Esino. Arti Grafiche Gentile, Fabriano: 27-39.

Sansa, R. 2003: Loro del verde. I boschi nello Stato Pontificio. Bologna, Clueb Editrice.

Scoppola, A. \& Caporali, C. 1998: Mesophilous woods with Fagus sylvatica L. of northern Latium (Tyrrhenian Central Italy): synecology and syntaxonomy. Plant Biosystems 132 (2): 151-168.

Taffetani, F. 2000: Serie di vegetazione del complesso geomorfologico del Monte dell'Ascensione (Italia centrale). Fitosociologia 37 (1): 93-151.

Taffetani, F., Zitti, S. \& Giannangeli, A. 2004: Vegetazione e paesaggio vegetale della dorsale di Cingoli (Appennino centrale, dorsale marchigiana). Fitosociologia 41 (2) suppl. 1: 3-81.

Theurillat, J.P. 1992: L'analyse du paysage vegetal en symphytocoenologie: ses niveaux et leurs domaines spatiaux. Bull. Ecol. 23 (1-2): 83-92.

Tomaselli, R. 1956: Introduzione allo studio della fitosociologia. Industria Poligrafica Lombarda, Milano.

Tutin, T.G., Heywood, V.H., Burges, N.A., Moore, D.M., Valentine, D.H., Walters, S.M. \& Webb, D.A. (eds.) (1964-80): Flora Europaea. Voll. 1-5. $1^{\text {st }}$ ed. Cambridge University Press.

Tutin, T.G., Burges, N.A., Chater, A.O., Edmonson, J.R., Heywood, V.H., Moore, D.M., Valentine, D.H., Walters, S.M. \& Webb, D.A. (eds.) (1993): Flora europaea. Vol. $1^{\text {st }} \cdot 2^{\text {nd }} e d$. Cambridge University Press.

Ubaldi, D. 1995: Tipificazione di syntaxa forestali appenninici e siciliani. Studi sul territorio Ann. Bot. (Roma) 51: 113-126.
Ubaldi, D. \& Speranza, M. (1985): Quelques hetraies du Fagion et du Laburno-Ostryon dans l'Apennin septentrional (Italie). Doc. Phytosoc. N.S. 9: 51-71.

Ubaldi, D., Zanotti, A.L., Puppi, G., Speranza, M. \& Corbetta, F. 1987: Sintassonomia dei boschi caducifogli mesofili dell'Italia peninsulare. Not. Fitosoc. 23: 31-62.

Van der Maarel, E., 1979: Trasformation of coverabundance values in phytosociology and its effects on community similarity. Vegetatio 39 : 97-144.

Vitanzi, A., Catorci, A. \& Properzi, S.: Caratterizzazione floristico-strutturale di alcuni boschi di faggio in condizioni di libera evoluzione (work in progress).

Weber, H.E., Moravec, J. \& Theurillat, J.P. 2002: International Code of Phytosociological Nomenclature. 3rd ed. Journal of Vegetation Science 11: 739-768.

Willner, W. 2002: Syntaxonomische Revision der südmitteleuropäischen Buchenwälder. Phytocoenologia 32 (3): 337-453.
Received 27. 7. 2009

Revision received 17. 3. 2010

Accepted 22. 3. 2010 


\section{APPENDIX:}

\section{sporadic taxa}

Table 3 - Carici digitatae-Ostryetum carpinifoliae

Rel. 1: Epipactis microphylla +; Rel. 2: Geranium robertianum +; Epipactis muelleri +; Asperula taurina subsp. taurina +; Primula veris subsp. suaveolens 1; Rel. 3: Rhamnus alpina subsp. alpina r; Silene italica subsp. italica r; Acer platanoides +; Cardamine enneaphyllos r; Rel. 4: Prenanthes purpurea +; Rel. 5: Helleborus foetidus subsp. foetidus r; Ulmus glabra +; Asplenium trichomanes subsp. quadrivalens r; Rel. 6: Digitalis lutea subsp. australis +; Scutellaria columnae subsp. columnae +; Viola alba subsp. dehnhardtii + ; Sorbus torminalis + ; Cardamine enneaphyllos +; Lilium martagon +; Anemone ranunculoides +; Saxifraga rotundifolia subsp. rotundifolia +; Ajuga reptans +; Geum urbanum +; Dactylis glomerata subsp. glomerata +; Rel. 7: Ligustrum vulgare +; Euonymus verrucosus +; Prunus avium subsp. avium +; Malus sylvestris +.

Table 5 - Lathyro veneti-Fagetum sylvaticae

Rel. 1: Ligustrum vulgare r; Rel. 3: Veronica officinalis +; Rel. 5: Listera ovata +; Rel. 6: Fraxinus excelsior subsp. excelsior +; Rel. 9: Corallorhiza trifida +; Rel. 11: Salix caprea +; Rel. 13: Cytisophyllum sessilifolium +; Stellaria nemorum (s.l.) +; Rel. 15: Vincetoxicum hirundinaria subsp. hirundinaria +; Asplenium onopteris +; Rel. 17: Hypericum montanum +; Rel. 18: Brachypodium rupestre +; Rel. 19: Phyllitis scolopendrium subsp. scolopendrium $+;$ Rel. 21: Staphylea pinnata +; Rel. 23: Festuca altissima +; Stellaria media subsp. media r; Rel. 24: Hordelymus europaeus +; Senecio ovatus subsp. alpestris +; Rel. 30: Chaerophyllum temulum +, Sambucus nigra +, Sedum cepaea +; Rel. 31: Primula veris subsp. suaveolens +; Rel. 32: Salvia glutinosa +; Rel. 33: Lapsana communis +, Campanula rapunculus +; Rel. 34: Ribes multiflorum +.

Table 7 - Solidagini-Fagetum sylvaticae

Rel. 1: Salvia glutinosa r; Rel. 2: Poa nemoralis subsp. nemoralis +; Viola alba subsp. dehnhardtii +; Fragaria vesca subsp. vesca r; Rel. 5: Laburnum alpinum +; Rel. 6: Crataegus laevigata +; Rel. 7:
Pulmonaria apennina r; Rel. 8: Fraxinus excelsior subsp. excelsior r; Sorbus aria subsp. aria r; Rel. 9: Athyrium filix-femina + ; Pyrola minor + ; Rel. 10: Aegopodium podagraria r; Polystichum setiferum+; Epipactis leptochila r; Adenostyles glabra subsp. glabra r; Rel. 11: Heracleum sphondylium subsp. ternatum r; Rel. 12: Campanula trachelium subsp. trachelium $\mathrm{r}$.

Table 8 - Cardamino kitaibelii-Fagetum sylvaticae typicum

Rel. 4: Helleborus foetidus subsp. foetidus +; Rel. 6: Veronica hederifolia subsp. hederifolia + , Cystopteris fragilis +; Rel. 7: Veronica hederifolia subsp. hederifolia +; Rel. 11: Dactylis glomerata subsp. glomerata +; Rel. 13: Dactylis glomerata subsp. glomerata +, Bunium bulbocastanum +, Rumex alpestris +; Rel. 14: Helleborus foetidus subsp. foetidus +; Rel. 21: Veronica chamaedrys subsp. chamaedrys 1; Luzula forsteri +; Rel. 25: Lamium galeobdolon subsp. montanum +;Veronica chamaedrys subsp. chamaedrys +; Rel. 26: Ostrya carpinifolia +; Rel. 28: Campanula rapunculus r; Rel. 29: Arabis turrita +; Emerus majus (s.l.) +; Rel. 30: Ribes uva-crispa r; Rel. 31: Lamium garganicum subsp. laevigatum +; Rel. 32: Laserpitium latifolium r; Rel. 33: Rumex alpestris +; Rel. 34: Cardamine heptaphylla +; Prunus avium subsp. avium +; Populus tremula +; Laserpitium latifolium +, Salix caprea 1; Rel. 37: Hordelymus europaeus +; Rel. 39: Milium effusum +; Lathyrus venetus +; Hedera helix subsp. helix +; Rel. 40: Arabis turrita r; Rel. 41: Alliaria petiolata +; Rel. 42: Valeriana tripteris subsp. tripteris $\mathrm{r}$.

Table 9 - Cardamino kitaibelii-Fagetum sylvaticae anemonetosum nemorosae

Rel. 1: Arum maculatum +; Polystichum setiferum +; Brachypodium sylvaticum subsp. sylvaticum +; Senecio alpinus + , Silene conica + , Aegopodium podagraria +; Rel. 3: Senecio alpinus 1, Silene conica +, Arabis turrita +, Veronica chamaedrys subsp. chamaedrys + , Chaerophyllum temulum +; Rel. 4: Epipactis muelleri + ; Fraxinus excelsior subsp. excelsior +; Rel. 5: Rubus idaeus +; Silene italica subsp. italica +; Rel. 7: Corydalis pumila +; Acer campestre +; Rel. 8: Clematis vitalba r; Rel. 9: Galium aparine +; Rel. 10: Galium aparine +; Rel. 13: Pteridium aquilinum subsp. aquilinum +; Rel. 14: Asplenium trichomanes subsp. quadrivalens + , Cotoneaster integerrimus 
+; Rel. 15: Asplenium trichomanes subsp. quadrivalens + , Primula veris subsp. suaveolens +; Rel. 17: Carpinus betulus +; Quercus pubescens subsp. pubescens r; Rel. 19: Potentilla micrantha +; Rel. 20: Crataegus laevigata r; Rel. 21: Ruscus hypoglossum +; Salix caprea 1; Rel. 22: Aegopodium podagraria + , Listera ovata r; Scrophularia scopolii +; Rel. 23: Polystichum lonchitis +; Platanthera chlorantha r; Salix caprea r; Rel. 25: Pulmonaria apennina r; Rhamnus alpina subsp. alpina + .

Table 10 - Cardamino kitaibelii-Fagetum sylvaticae corallorhizetosum trifidae

Rel. 4: Laserpitium latifolium +; Solidago virgaurea subsp. virgaurea +; Rel. 5: Valeriana tripteris subsp. tripteris +; Rel. 6: Cystopteris fragilis +; Rel. 7: Anemone apennina subsp. apennina +; Epipactis leptochila +; Rel. 10: Veronica officinalis $+;$ Ribes uva-crispa +; Rel. 11: Paris quadrifolia +; Rel. 12: Orthilia secunda +; Rel. 13: Fragaria vesca subsp. vesca +, Sesleria nitida (s.l.) r; Rel. 14: Daphne mezereum +; Rosa arvensis +; Geum urbanum r; Rel.15: Festuca altissima + ; Brachypodium sylvaticum subsp. sylvaticum r; Fragaria vesca subsp. vesca r; Rel. 17: Veronica montana +; Rel. 20: Ranunculus ficaria subsp. bulbifer +; Rel. 21: Epipactis muelleri +; Rel. 22: Ranunculus ficaria subsp. bulbifer +; Rel. 24: Rubus idaeus + , Crocus vernus subsp. vernus + ; Rel. 25: Lamium galeobdolon subsp. montanum +; Rel. 29: Luzula sylvatica subsp. sylvatica +; Rel. 30: Veronica hederifolia subsp. hederifolia + ; Calamintha nepeta subsp. nepeta +; Dactylis glomerata subsp. glomerata + .

Table 11 - Impoverished facies of Cardamino kitaibelii-Fagetum sylvaticae

Rel. 1: Hypericum montanum +; Potentilla micrantha + ; Rel. 2: Juniperus communis subsp. communis +, Sedum cepaea +; Rel. 3: Gagea lutea +; Veronica chamaedrys subsp. chamaedrys + , Moehringia trinervia +, Campanula micrantha +; Rel. 4: Polystichum aculeatum +; Rel. 6: Sambucus nigra +; Geranium robertianum +; Ilex aquifolium +; Rel. 7: Brachypodium sylvaticum subsp. sylvaticum + ; Rosa arvensis + ; Rel. 8: Aremonia agrimonoides subsp agrimonoides +; Hepatica nobilis +; Rel. 9: Geranium nodosum +; Euonymus latifolius +; Rel. 10: Aegopodium podagraria + ; Cardamine heptaphylla + ; Sorbus aucuparia subsp. aucuparia + .
Table 12 - Aceretum obtusati-pseudoplatani

Rel. 1: Allium ursinum subsp. ursinum + ; Lathyrus vernus subsp. vernus +; Thalictrum aquilegifolium subsp. aquilegifolium +; Polygonatum verticillatum r; Viola alba subsp. dehnhardtii +; Primula vulgaris subsp. vulgaris r; Silene conica r, Ranunculus ficaria subsp. bulbifer 1, Sambucus nigra +; Rel. 2: Senecio ovatus subsp. alpestris +; Rel. 3: Aegopodium podagraria +; Paris quadrifolia +; Laburnum alpinum +; Polystichum aculeatum +; Festuca altissima +; Rel. 5: Sesleria nitida (s.l.) 1, Ceterach officinarum subsp. officinarum +; Dryopteris filix-mas +; Rel. 6: Bromus ramosus +; Valeriana tripteris subsp. tripteris +; Hieracium murorum +; Geum urbanum +; Primula veris subsp. suaveolens +; Rel. 7: Heracleum sphondylium subsp. ternatum +; Ribes multiflorum +; Fraxinus ornus subsp. ornus +; Fragaria vesca subsp. vesca +; Lamium maculatum +; Rel. 8: Rosa canina + , Elymus caninus subsp. caninus +; Rel. 9: Actaea spicata +; Epipactis leptochila +; Alliaria petiolata +; Lonicera caprifolium +; Moehringia trinervia + , Eranthis hyemalis 1, Chaerophyllum aureum + , Silene italica subsp. nemoralis + . 
Table 1: Bioclimatic belts of the study area.

Tabela 1: Bioklimatski pasovi obravnvanega območja.

\begin{tabular}{|c|c|c|c|c|c|c|c|c|c|c|c|c|}
\hline 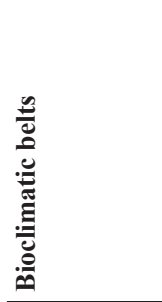 & 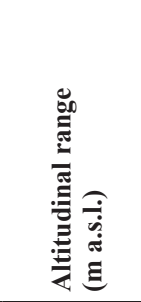 & 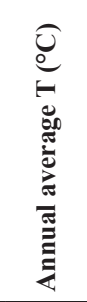 & 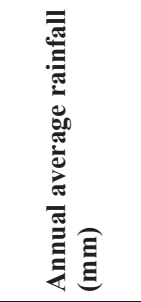 & 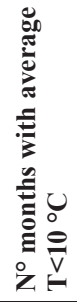 & 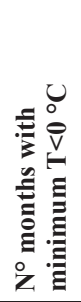 & 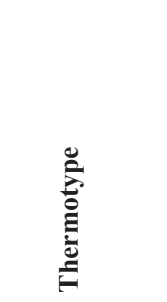 & 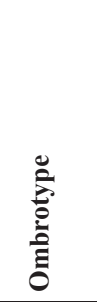 & 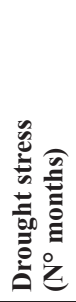 & ติ & 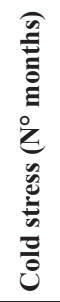 & $\bigcup^{2}$ & 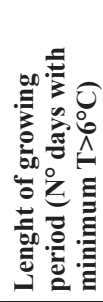 \\
\hline $\begin{array}{l}\text { Upper Meso- } \\
\text { temperate }\end{array}$ & $600-1000$ & $11-13$ & $850-1100$ & 6 & 1 & $\begin{array}{l}\text { Upper Meso- } \\
\text { temperate }\end{array}$ & $\begin{array}{l}\text { Lower } \\
\text { humid }\end{array}$ & - & $0-20$ & $6-7$ & $400-500$ & $190-220$ \\
\hline $\begin{array}{l}\text { Lower Supra- } \\
\text { temperate }\end{array}$ & $1000-1400$ & $9-11$ & $1100-1300$ & 6 & 3 & $\begin{array}{l}\text { Lower Supra- } \\
\text { temperate }\end{array}$ & $\begin{array}{l}\text { Upper } \\
\text { humid }\end{array}$ & - & - & $7-8$ & $500-600$ & $160-190$ \\
\hline $\begin{array}{l}\text { Upper Supra- } \\
\text { temperate }\end{array}$ & $1400-1800$ & $7-9$ & $1300-1500$ & 8 & 4 & $\begin{array}{l}\text { Upper Supra- } \\
\text { temperate }\end{array}$ & $\begin{array}{l}\text { Lower } \\
\text { hyper- } \\
\text {-humid }\end{array}$ & - & - & $8-9$ & $600-700$ & $120-150$ \\
\hline
\end{tabular}

Table 2: PCA matrix of environmental parameters classes.

Table 2: PCA matrica razredov rastiščnih dejavnikov.

\begin{tabular}{lcc}
\hline & & Classes \\
\hline Geological substrata & Limestone & 1 \\
& Calcari Diasprini Formation & 2 \\
& Sandstone & 3 \\
\hline \multirow{2}{*}{ Altitude (m a.s.l.) } & $600-1000 \mathrm{~m}$ & 1 \\
& $1000-1400 \mathrm{~m}$ & 2 \\
& $1400-1800 \mathrm{~m}$ & 3 \\
\hline \multirow{2}{*}{ Slope $\left(^{\circ}\right)$} & $>45^{\circ}$ & 1 \\
& $15-45^{\circ}$ & 2 \\
Morphology & $<15^{\circ}$ & 3 \\
\hline
\end{tabular}


Table 3 (Tabela 3): Carici digitatae-Ostryetum carpinifoliae Catorci, Gatti et Sparvoli 2003.

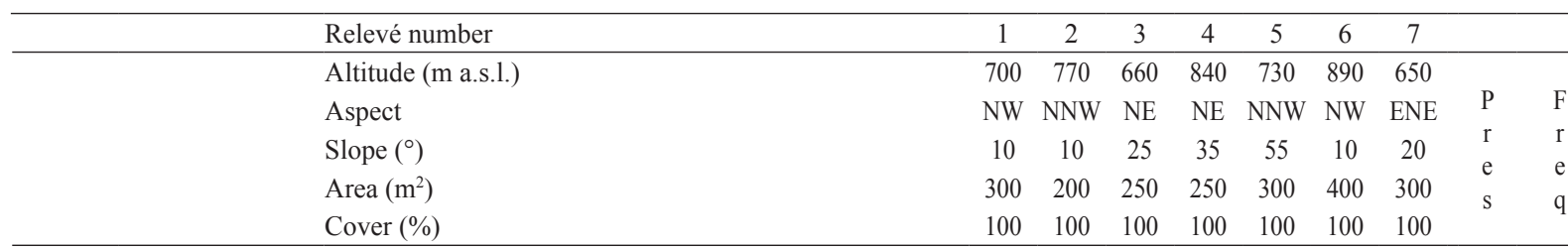

$\begin{array}{lllllllllllll}H & \text { caesp } & \text { Eurasiat. } & \text { Carex digitata } & 1 & 1 & 2 & 1 & 1 & . & 1 & 6 & 86\end{array}$

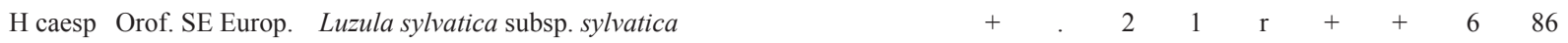

Grhiz Eurimedit. Ruscus aculeatus $\quad$. + + . $\quad+\quad$. $\quad+\quad+\quad 4 \quad 57$

P scap Europ.-Caucas. Tilia platyphyllos subsp.platyphyllos $\quad$. . . $\quad+\quad$. $\quad \begin{array}{ccccc}1 & 1 & 1 & 4 & 57\end{array}$

P caesp S Europ.-Sudsib. Staphylea pinnata $\quad$. . . . . + . $+{ }^{+}+3$

Differential species of the sub-alliance Laburno anagyroidis-Ostryenion carpinifoliae

P caesp S Europ.-Sudsib. Laburnum anagyroides subsp. anagyroides $\quad \begin{aligned} & 1 \\ & \text { S }\end{aligned}$

H scap Paleotemp. Sanicula europaea $\quad+\quad+\quad+\quad \begin{array}{ccccc}1 & 3 & + & 1 & 6\end{array}$

G bulb Orof. C Europ. Lilium bulbiferum subsp. croceum $\quad+\quad+\quad$ r $\quad$ r $\quad$ r $\quad . \quad$. $\quad 5$. 71

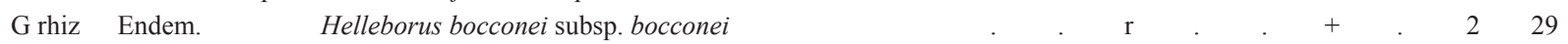

T scap Endem. Melampyrum italicum

Charact. species of the alliance Carpinion orientalis

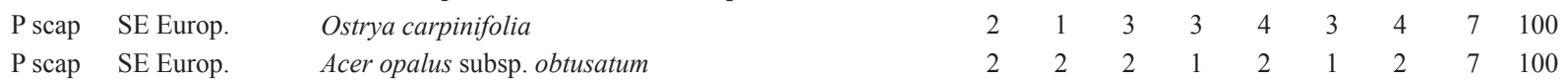

NP C Europ. Emerus majus (s.1.) . . $\quad+\quad+\quad+\quad+\quad+\quad 5+71$

Charact. species of the order Quercetalia pubescenti-petraeae

P caesp Subatlant. Daphne laureola

G bulb S Europ. Cyclamen hederifolium subsp. hederifolium

P scap S Europ.-Sudsib. Fraxinus ornus subsp. ornus

G rhiz S Europ.-Sudsib. Lathyrus venetus

P caesp S Europ.-Sudsib. Cornus mas

P caesp Paleotemp. Sorbus aria subsp. aria

G rhiz Circumbor. Hepatica nobilis

H scap C Europ. Melittis melissophyllum subsp. melissophyllum

P caesp Europ.-Caucas. Lonicera xylosteum

P caesp Medit. mont. Euonymus latifolius

G rhiz Eurasiat. Cephalanthera longifolia

P caesp C Europ. Viburnum lantana

P caesp C Europ. Crataegus laevigata

P caesp Eurasiat. Euonymus europaeus

G rhiz Paleotemp. Epipactis helleborine (s.1.)

P caesp SE Europ. Quercus pubescens subsp. pubescens

P scap N Eurimedit. Quercus cerris

P scap Eurimedit. Sorbus domestica

$\mathrm{H}$ ros Paleotrop. Polypodium interjectum

Transgressive species from the order Fagetalia sylvaticae

H scap Eurosib.

Viola reichenbachiana

H caesp Paleotemp. Melica uniflora

P scap C Europ. Fagus sylvatica subsp. sylvatica

G rhiz Eurasiat. Neottia nidus-avis

P scap Europ.-Caucas. Carpinus betulus

H caesp Europ.-Caucas. Festuca heterophylla

G bulb Europ.-Caucas. Scilla bifolia

H scap Paleotemp. Campanula trachelium subsp. trachelium

$\begin{array}{ccccccccc}1 & + & + & + & 1 & . & + & 6 & 86 \\ . & + & + & + & + & + & + & 6 & 86 \\ 2 & 3 & 2 & 2 & . & 1 & . & 5 & 71 \\ + & + & \text { r } & \text { r } & . & . & 2 & 5 & 71 \\ 4 & 3 & . & . & 1 & + & 1 & 5 & 71 \\ + & . & + & . & + & + & 1 & 5 & 71 \\ . & + & . & + & + & + & . & 4 & 57 \\ . & + & \text { r } & \text { r } & . & + & . & 4 & 57\end{array}$




\begin{tabular}{|c|c|c|c|c|c|c|c|c|c|c|c|}
\hline & & Relevé number & 1 & 2 & 3 & 4 & 5 & 6 & 7 & Pres & $\overline{\text { Freq }}$ \\
\hline H scap & Endem. & Pulmonaria apennina & . & + & . & $\mathrm{r}$ & . & + & 2 & 4 & 57 \\
\hline H scap & Europ.-Caucas. & Lactuca muralis & . & . & . & + & 1 & + & 2 & 4 & 57 \\
\hline G rhiz & Circumbor. & Anemone nemorosa & . & + & . & + & . & + & . & 3 & 43 \\
\hline P scap & Europ.-Caucas. & Acer pseudoplatanus & . & . & + & . & 1 & . & + & 3 & 43 \\
\hline G rhiz & Europ.-Caucas. & Galium odoratum & + & 1 & . & . & 1 & . & . & 3 & 43 \\
\hline P scap & Europ.-Caucas. & Acer campestre & . & + & . & . & . & . & + & 2 & 29 \\
\hline G bulb & Paleotemp. & Dactylorhiza maculata subsp. fuchsii & . & . & + & $\mathrm{r}$ & . & . & . & 2 & 29 \\
\hline G bulb & Eurosib. & Gagea lutea & . & . & + & . & . & + & . & 2 & 29 \\
\hline G rhiz & C Europ. & Cardamine bulbifera & . & + & . & . & . & + & . & 2 & 29 \\
\hline G rhiz & C Europ. & Euphorbia dulcis & . & . & $\mathrm{r}$ & . & + & . & . & 2 & 29 \\
\hline P caesp & Eurimedit. & Ilex aquifolium & . & . & . & + & . & 1 & . & 2 & 29 \\
\hline G rhiz & Europ.-Caucas. & Mercurialis perennis & . & . & . & + & . & + & . & 2 & 29 \\
\hline H scap & Orof. Eurasiat. & Salvia glutinosa & . & . & . & . & 1 & . & 2 & 2 & 29 \\
\hline H caesp & Eurasiat. & Bromus ramosus & . & . & . & . & . & + & 2 & 2 & 29 \\
\hline Ch suffr & Europ.-Caucas. & Euphorbia amygdaloides subsp. amygdaloides & . & . & . & . & . & + & + & 2 & 29 \\
\hline \multirow[t]{2}{*}{ G rhiz } & Eurasiat. & Cephalanthera rubra & . & . & . & . & . & . & 2 & 1 & 14 \\
\hline & & Character species of the class Querco-Fagetea & & & & & & & & & \\
\hline P lian & Eurimedit. & Hedera helix subsp. helix & 1 & 1 & 1 & + & 1 & 1 & 1 & 7 & 100 \\
\hline NP & S Medit.-Subatl. & Rosa arvensis & + & + & + & + & + & . & + & 6 & 86 \\
\hline P caesp & Europ.-Caucas. & Corylus avellana & 2 & 2 & 1 & + & 1 & + & . & 6 & 86 \\
\hline $\mathrm{H}$ ros & Europ.-Caucas. & Primula vulgaris subsp. vulgaris & + & . & + & + & + & + & + & 6 & 86 \\
\hline G bulb & Europ.-Caucas. & Galanthus nivalis subsp. nivalis & + & + & + & . & $\mathrm{r}$ & + & . & 5 & 71 \\
\hline G rhiz & SE Europ. & Anemone apennina subsp. apennina & 1 & + & 1 & . & + & . & + & 5 & 71 \\
\hline $\mathrm{H}$ rept & Eurosib. & Fragaria vesca subsp. vesca & + & . & + & + & . & + & + & 5 & 71 \\
\hline NP & N Eurimedit. & Rubus hirtus & . & + & + & + & + & 1 & . & 5 & 71 \\
\hline H caesp & Paleotemp. & Brachypodium sylvaticum subsp. sylvaticum & . & . & + & + & $\mathrm{r}$ & . & 1 & 4 & 57 \\
\hline G rhiz & Eurimedit. & Cephalanthera damasonium & . & . & $\mathrm{r}$ & + & $\mathrm{r}$ & . & 2 & 4 & 57 \\
\hline H scap & Eurasiat. & Cruciata glabra subsp. glabra & . & . & $\mathrm{r}$ & . & + & . & + & 3 & 43 \\
\hline H scap & Eurosib. & Hieracium murorum & + & . & + & . & . & . & 1 & 3 & 43 \\
\hline H scap & Circumbor. & Solidago virgaurea subsp. virgaurea & . & . & $\mathrm{r}$ & $\mathrm{r}$ & . & 1 & . & 3 & 43 \\
\hline T scap & N Medit. & Cardamine graeca & + & + & . & . & . & + & . & 3 & 43 \\
\hline G rad & Eurimedit. & Tamus communis & + & . & . & . & . & + & 2 & 3 & 43 \\
\hline $\mathrm{H}$ caesp & Eurimedit. & Luzula forsteri & + & . & . & . & . & + & . & 2 & 29 \\
\hline P lian & S Europ.-Sudsib. & Lonicera caprifolium & . & . & + & + & . & . & . & 2 & 29 \\
\hline \multirow[t]{2}{*}{ G rhiz } & Circumbor. & Polystichum setiferum & . & . & . & $\mathrm{r}$ & . & + & . & 2 & 29 \\
\hline & & Accompanying taxa & & & & & & & & & \\
\hline$P$ caesp & Paleotemp. & Crataegus monogyna & . & . & + & . & + & + & + & 4 & 57 \\
\hline P scap & Stenomedit. & Quercus ilex subsp. ilex & 1 & 1 & + & . & . & . & . & 3 & 43 \\
\hline $\mathrm{H}$ ros & Cosmopol. & Asplenium adiantum-nigrum subsp. adiantum-nigrum & + & + & . & . & . & . & . & 2 & 29 \\
\hline$P$ caesp & Orof. SW Europ. & Cytisophyllum sessilifolium & + & . & $\mathrm{r}$ & . & . & . & . & 2 & 29 \\
\hline \multirow[t]{2}{*}{ P caesp } & Circumbor. & Juniperus communis subsp. communis & . & . & . & . & + & . & + & 2 & 29 \\
\hline & & Sporadic taxa & 1 & 4 & 4 & 1 & 3 & 11 & 4 & & \\
\hline
\end{tabular}


Table 4: Plant species frequency in different altitudinal ranges within the present study: black - very frequent; grey and dark grey - rare or not frequent; white - absent.

Tabela 4: Frekvenca rastlinskih vrst v različnih višinskih pasovih: črna - zelo pogosto, siva in temno siva - redko; bela - vrsta ni prisotna.

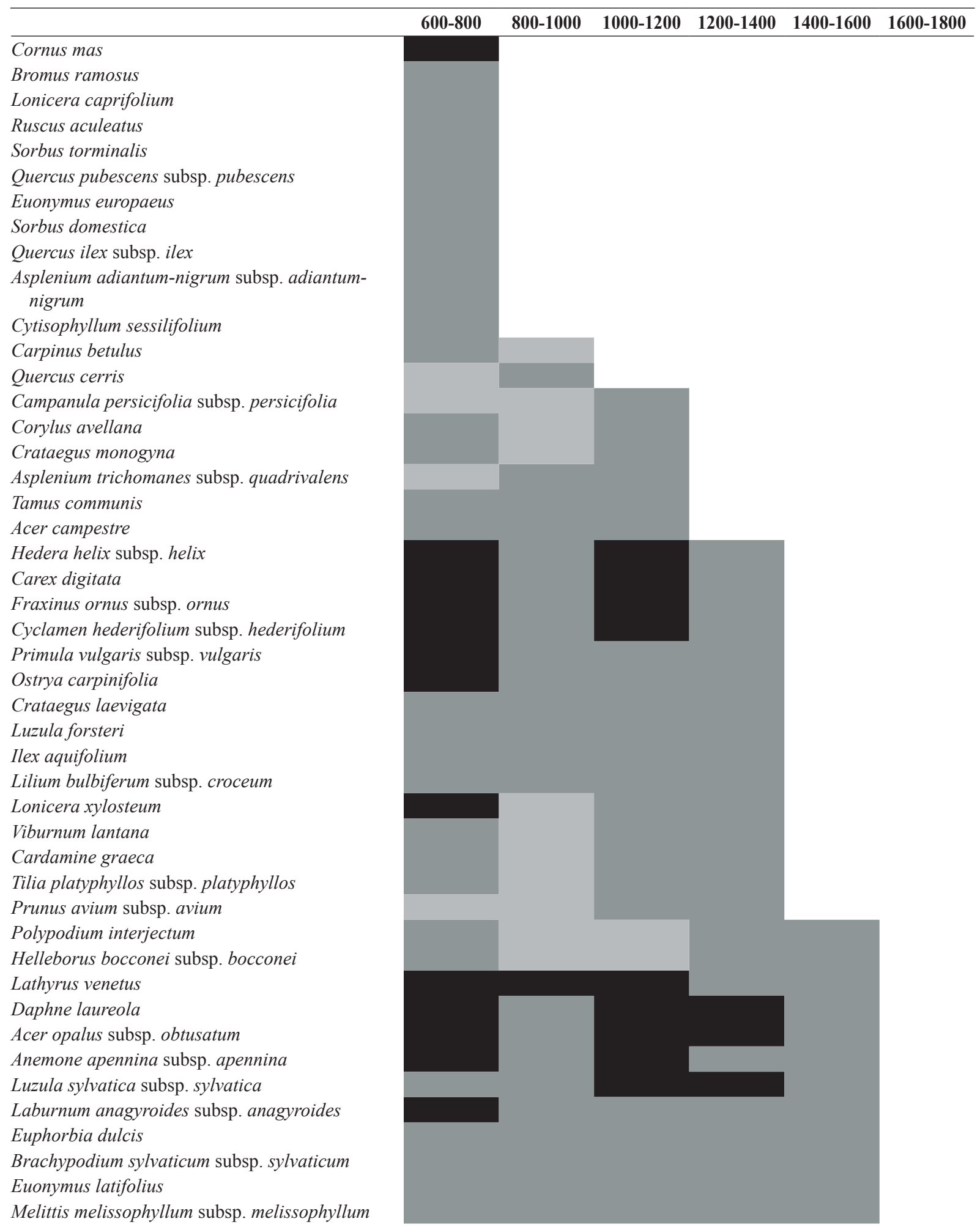




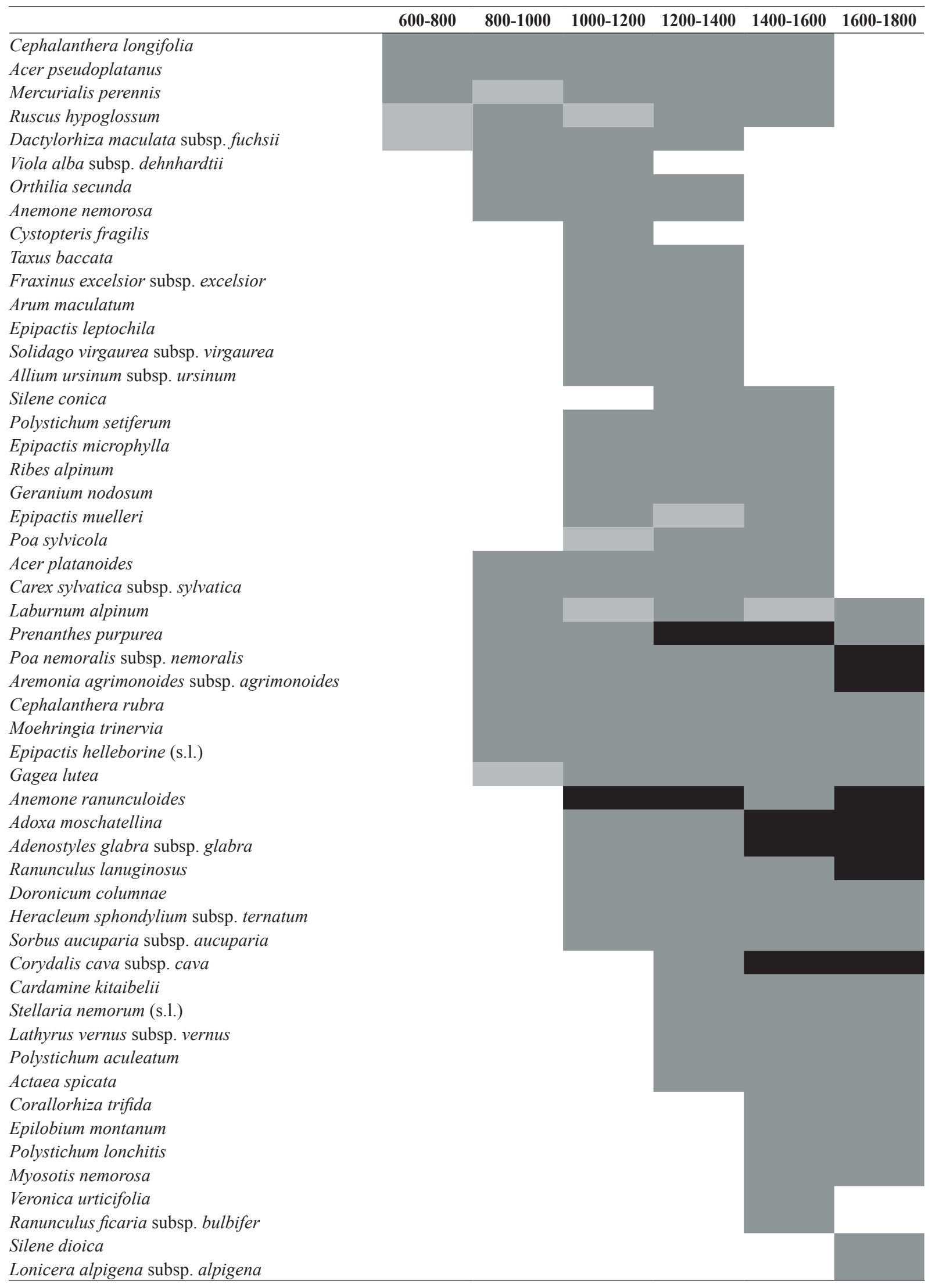


Table 5 (Tabela 5): Lathyro veneti-Fagetum sylvaticae Biondi, Casavecchia, Pinzi, Allegrezza et Baldoni 2002 lathyretosum veneti Biondi, Casavecchia, Pinzi, Allegrezza et Baldoni 2002, hieracietosum murori subass. nova and Galium aparine variant.

\begin{tabular}{llcccccccccccc}
\hline & Relevé number & 1 & 2 & $3^{*}$ & 4 & 5 & 6 & 7 & 8 & 9 & 10 & 11 & 12 \\
\hline Altitude (m a.s.1.) & 750 & 840 & 1080 & 1190 & 1230 & 1290 & 990 & 1085 & 1105 & 1100 & 1160 & 911 \\
& Aspect & $\mathrm{NE}$ & $\mathrm{N}$ & $\mathrm{N}$ & $\mathrm{NE}$ & $\mathrm{N}$ & $\mathrm{NNE}$ & $\mathrm{N}$ & $\mathrm{E}$ & $\mathrm{E}$ & $\mathrm{E}$ & $\mathrm{NE}$ & $\mathrm{NE}$ \\
Slope $\left({ }^{\circ}\right)$ & 20 & 30 & 40 & 20 & 30 & 35 & 15 & 5 & 10 & 10 & 35 & 15 \\
& Area $\left(\mathrm{m}^{2}\right)$ & 300 & 400 & 400 & 400 & 400 & 400 & 250 & 250 & 250 & 200 & 300 & 400 \\
& Cover $(\%)$ & 100 & 100 & 100 & 100 & 100 & 100 & 100 & 100 & 100 & 100 & 100 & 100 \\
\hline
\end{tabular}

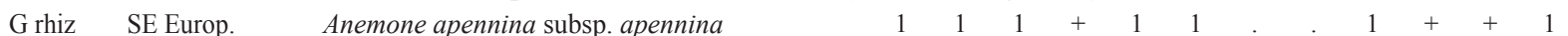

G rhiz S Europ.-Sudsib. Lathyrus venetus

G bulb S Europ. Cyclamen hederifolium subsp. hederifolium

$\mathrm{H}$ ros $\quad$ Eurimedit. Viola alba subsp. dehnhardtii

G rhiz Circumbor. Anemone nemorosa

G rhiz Eurasiat. Polygonatum multiflorum

\begin{tabular}{lll} 
& & \multicolumn{1}{c}{ Species of the Galium aparine variant } \\
H scap & Endem. & Digitalis lutea subsp. australis \\
NP & Eurosib. & Ribes alpinum \\
T scap & Eurasiat. & Galium aparine \\
G rhiz & Orof. SE-Europ. & Doronicum columnae
\end{tabular}

Diff. species of the subass. hieracietosum murorum

$\begin{array}{lll}\text { NP } & \text { S Medit.-Subatl. } & \text { Rosa arvensis } \\ \text { H caesp } & \text { Eurasiat. } & \text { Carex digitata } \\ \text { H scap } & \text { Eurosib. } & \text { Hieracium murorum } \\ \text { H caesp } & \text { Europ.-E Asiat. } & \text { Carex sylvatica subsp. sylvatica } \\ \text { H ros } & \text { Eurimedit. } & \text { Potentilla micrantha } \\ \text { H caesp } & \text { Eurimedit. } & \text { Luzula forsteri } \\ \text { G bulb } & \text { Eurosib. } & \text { Platanthera chlorantha }\end{array}$

\begin{tabular}{|cccccccccccc}
$\mathrm{r}$ & + & + & + & + & + & + & + & + & + & + &. \\
1 & 1 & + &. & + & + & 1 & 1 & 1 & + & + & + \\
$\mathrm{r}$ &. & + &. & + & + & $\mathrm{r}$ & + & $\mathrm{r}$ & $\mathrm{r}$ &. &. \\
+ & $\cdot$ & + &. &. & + & + &. &. & + &. &. \\
. & $\mathrm{r}$ & + & + &. &. & + &. & + &. & + & + \\
. & $\mathrm{r}$ & + &. & $\mathrm{r}$ &. & + &. &. & + & $\mathrm{r}$ &. \\
$\mathrm{r}$ &. & $\mathrm{r}$ &. & $\mathrm{r}$ &. &. &. &. &. &. & + \\
\hline
\end{tabular}

$\begin{array}{lll}\text { P scap } & \text { SE Europ. } & \text { Acer opalus subsp. obtusatum } \\ \text { P caesp } & \text { Subatlant. } & \text { Daphne laureola } \\ \text { G rhiz } & \text { Europ.-Caucas. } & \text { Anemone ranunculoides } \\ \text { H scap } & \text { Endem. } & \text { Pulmonaria apennina } \\ \text { H scap } & \text { Europ.-Caucas. } & \text { Ranunculus lanuginosus } \\ \text { G rhiz } & \text { Eurimedit. } & \text { Ruscus hypoglossum } \\ \text { G rhiz } & \text { Orof. SE Europ. } & \text { Cardamine kitaibelii }\end{array}$

Orof. SE Europ. Cardamine kitaibelii

\begin{tabular}{|c|c|c|c|c|c|c|c|c|c|c|c|c|c|}
\hline & & & & & & & & & & & & & \\
\hline P scap & C Europ. & Fagus sylvatica subsp. sylvatica & 3 & 5 & 4 & 5 & 5 & 5 & 5 & 5 & 5 & 5 & 5 \\
\hline H scap & Paleotemp. & Sanicula europaea & + & 2 & . & 1 & 1 & + & 1 & + & + & $\mathrm{r}$ & + \\
\hline H scap & Eurosib. & Viola reichenbachiana & + & + & + & 1 & + & + & + & + & + & + & 1 \\
\hline $\mathrm{H}$ caesp & Europ.-Caucas. & Festuca heterophylla & + & + & + & + & + & + & . & + & + & + & + \\
\hline $\mathrm{H}$ caesp & Paleotemp. & Melica uniflora & + & + & . & . & . & . & + & $\mathrm{r}$ & + & . & $\mathrm{r}$ \\
\hline H scap & Paleotemp. & Campanula trachelium subsp. trachelium & $\mathrm{r}$ & . & + & . & . & . & + & + & + & $\mathrm{r}$ & + \\
\hline H scap & Europ.-Caucas. & Lactuca muralis & . & + & + & + & $\mathrm{r}$ & . & + & . & . & . & $\mathrm{r}$ \\
\hline G rhiz & Eurasiat. & Neottia nidus-avis & + & $\mathrm{r}$ & + & . & 1 & + & $\mathrm{r}$ & $\mathrm{r}$ & $\mathrm{r}$ & . & $\mathrm{r}$ \\
\hline G bulb & Eurasiat. & Lilium martagon & 1 & + & + & + & $\mathrm{r}$ & . & + & + & + & $\mathrm{r}$ & $\mathrm{r}$ \\
\hline G bulb & Europ.-Caucas. & Scilla bifolia & . & + & . & + & . & + & + & . & + & . & + \\
\hline$P$ caesp & Paleotemp. & Sorbus aria subsp. aria & . & + & + & . & $\mathrm{r}$ & . & + & . & + & + & + \\
\hline G rhiz & C Europ. & Euphorbia dulcis & + & + & . & . & . & . & + & + & + & . & $\mathrm{r}$ \\
\hline
\end{tabular}




\begin{tabular}{cccccccccccccccccccccccccccc}
\hline 13 & 14 & 15 & 16 & 17 & 18 & 19 & 20 & 21 & 22 & 23 & 24 & 25 & 26 & 27 & 28 & 29 & 30 & 31 & 32 & 33 & 34 & 35 & 36 & 37 & \\
\hline 980 & 970 & 1100 & 1125 & 1150 & 1170 & 640 & 930 & 910 & 1250 & 800 & 1280 & 1100 & 1230 & 1290 & 898 & 960 & 1030 & 1034 & 937 & 1270 & 1200 & 1265 & 1400 & 1400 & & \\
ENE & NE & N & NNE & NW & WSW & N & NW & NW & ENE & NE & E & N & N & NNE NNW & S & S & SW & SE & NW & ENE & NE & N & E & P & F \\
45 & 15 & 45 & 20 & 30 & 45 & 20 & 45 & 30 & 50 & 20 & 40 & 20 & 20 & 55 & 40 & 45 & 25 & 30 & 45 & 22 & 20 & 15 & 40 & 35 & $\mathrm{r}$ & $\mathrm{r}$ \\
300 & 400 & 200 & 200 & 200 & 400 & 300 & 250 & 350 & 350 & 300 & 300 & 250 & 250 & 200 & 350 & 300 & 300 & 200 & 300 & 200 & 400 & 400 & 500 & 400 & $\mathrm{~S}$ & $\mathrm{e}$ \\
98 & 100 & 98 & 100 & 100 & 98 & 100 & 99 & 100 & 100 & 100 & 100 & 98 & 100 & 100 & 98 & 98 & 100 & 98 & 98 & 90 & 100 & 100 & 100 & 100 & $\mathrm{q}$ \\
\hline
\end{tabular}

\section{ation lathyretosum veneti}

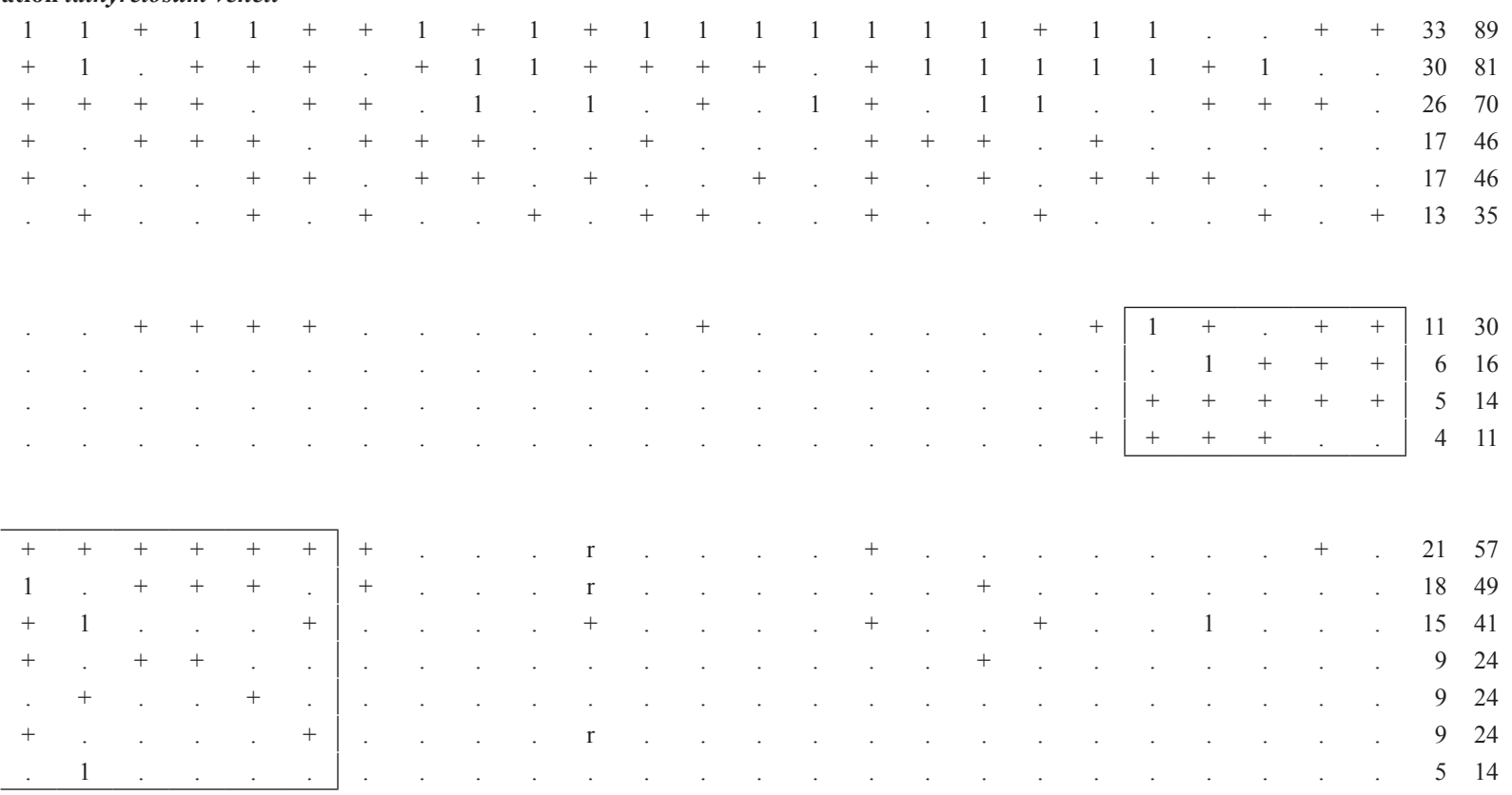

$$
\begin{aligned}
& \begin{array}{llllllllllllllllllllllllllllllllllllllllllllll}
5 & 3 & 4 & 5 & 5 & 5 & 3 & 4 & 4 & 5 & 5 & 5 & 4 & 5 & 5 & 5 & 5 & 5 & 5 & 5 & 3 & 5 & 4 & 5 & 4 & 37 & 100
\end{array}
\end{aligned}
$$

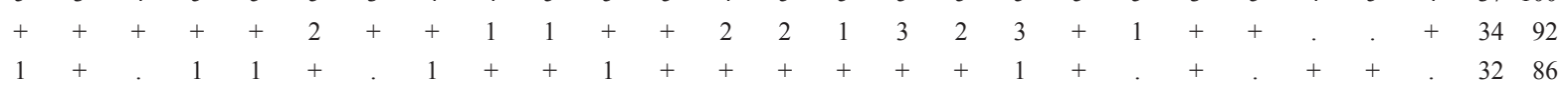

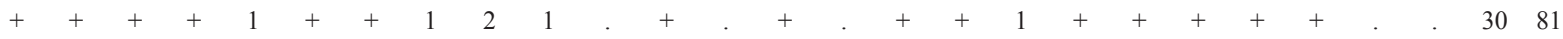

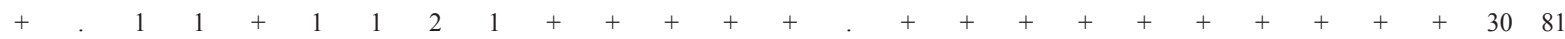

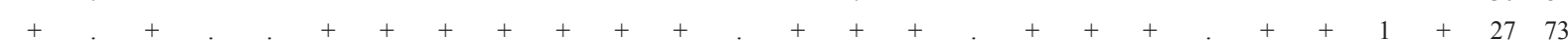

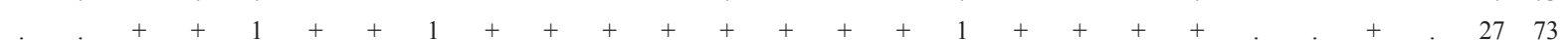

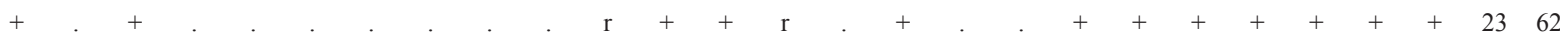

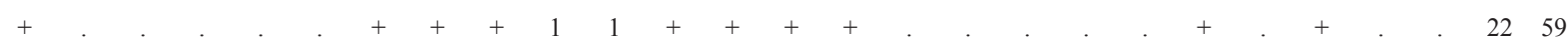

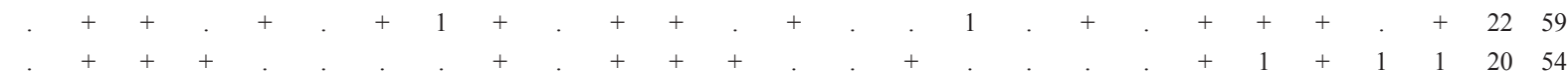

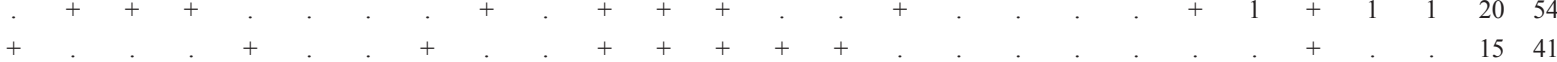




\begin{tabular}{|c|c|c|c|c|c|c|c|c|c|c|c|c|c|c|}
\hline & & Relevé number & 1 & 2 & $3 *$ & 4 & 5 & 6 & 7 & 8 & 9 & 10 & 11 & 12 \\
\hline Ch suffr & Europ.-Caucas. & Euphorbia amygdaloides subsp. amygdaloides & $\mathrm{r}$ & $\mathrm{r}$ & . & . & . & . & . & . & . & . & . & . \\
\hline G rhiz & C Europ. & Cardamine bulbifera & $\mathrm{r}$ & . & . & . & . & . & . & . & . & . & . & . \\
\hline H scap & Europ.-Caucas. & Prenanthes purpurea & . & $\mathrm{r}$ & . & . & . & . & $\mathrm{r}$ & + & . & . & . & . \\
\hline G bulb & Paleotemp. & Dactylorhiza maculata subsp. fuchsii & . & . & . & + & . & . & . & + & + & + & + & . \\
\hline H scap & Orof. S Europ. & Saxifraga rotundifolia subsp. rotundifolia & . & . & . & . & . & & . & . & . & . & . & . \\
\hline H caesp & Circumbor. & Poa nemoralis subsp. nemoralis & . & . & + & . & + & . & . & . & . & + & $\mathrm{r}$ & . \\
\hline$P$ caesp & Eurimedit. & Ilex aquifolium & + & . & . & . & . & . & . & . & . & . & . & . \\
\hline G bulb & Eurosib. & Gagea lutea & . & . & . & + & . & . & . & + & . & . & . & + \\
\hline P scap & Europ.-Caucas. & Acer pseudoplatanus & . & . & . & . & . & . & . & . & . & . & . & . \\
\hline T scap & Subcosmop. & Geranium robertianum & . & . & . & . & . & . & . & . & . & $\mathrm{r}$ & . & . \\
\hline P scap & Paleotemp. & Taxus baccata & . & . & . & . & . & . & . & . & . & . & + & . \\
\hline G rhiz & Eurasiat. & Cephalanthera rubra & . & . & . & . & + & . & . & + & . & + & + & + \\
\hline P scap & Europ.-Caucas. & Acer platanoides & . & . & . & . & . & . & . & . & . & . & . & . \\
\hline P scap & Pont. & Prunus avium subsp. avium & . & + & . & + & + & + & . & . & . & . & + & . \\
\hline G rhiz & Circumbor. & Polystichum setiferum & . & . & . & . & . & . & . & . & . & . & . & . \\
\hline G rhiz & Europ.-Caucas. & Mercurialis perennis & + & . & . & . & . & . & . & . & . & . & . & . \\
\hline G bulb & Eurasiat. & Allium ursinum subsp. ucrainicum & 1 & + & . & . & . & . & . & + & & . & . & . \\
\hline G rhiz & SE Europ. & Cardamine enneaphyllos & $\mathrm{r}$ & . & . & . & . & . & . & . & . & . & . & . \\
\hline G rhiz & C Europ. & Arum maculatum & . & . & . & . & . & . & $\mathrm{r}$ & $\mathrm{r}$ & . & . & . & . \\
\hline Ch rept & Circumbor. & Orthilia secunda & . & . & . & . & $\mathrm{r}$ & + & . & . & + & . & . & . \\
\hline G rhiz & Subcosmop. & Dryopteris filix-mas & $\mathrm{r}$ & . & . & . & . & . & . & . & . & . & . & . \\
\hline G rhiz & Paleotemp. & Epipactis leptochila & . & . & $\mathrm{r}$ & + & + & . & . & . & . & . & . & . \\
\hline H caesp & Eurasiat. & Bromus ramosus & . & . & . & . & . & . & . & . & . & . & . & . \\
\hline P scap & Europ.-Caucas. & Tilia platyphyllos subsp. platyphyllos & . & . & . & . & . & . & . & . & . & . & . & . \\
\hline Ch scap & Europ.-Caucas. & Stellaria holostea subsp. holostea & . & . & . & . & . & . & . & . & . & . & . & . \\
\hline P scap & Europ.-Caucas. & Carpinus betulus & + & 1 & + & . & . & . & . & . & . & . & . & 1 \\
\hline G bulb & Eurasiat. & Allium ursinum subsp. ursinum & . & . & . & . & . & . & . & . & . & . & . & . \\
\hline H scap & Paleotemp. & Heracleum sphondylium subsp. ternatum & . & . & . & . & . & . & . & . & . & $\mathrm{r}$ & . & . \\
\hline G rhiz & Eurasiat. & Actaea spicata & . & . & . & . & . & . & . & . & . & . & . & . \\
\hline G rhiz & C e S Europ. & Epipactis muelleri & . & . & . & . & . & . & . & . & . & . & . & . \\
\hline H scap & Orof. S Europ. & Aconitum lycoctonum & $\cdot$ & . & $\cdot$ & . & . & . & . & . & . & $\cdot$ & . & . \\
\hline G rhiz & N Medit. mont. & Geranium nodosum & . & . & . & . & . & . & . & . & $\mathrm{r}$ & $\mathrm{r}$ & . & . \\
\hline$P$ caesp & Europ. & Sorbus aucuparia subsp. aucuparia & . & . & . & . & . & . & . & . & . & . & . & . \\
\hline H scap & NE Medit. mont. & Adenostyles glabra subsp. glabra & . & . & . & . & . & . & . & . & . & . & . & . \\
\hline$P$ caesp & Orof. S Europ. & Laburnum alpinum & . & . & . & . & + & . & + & . & . & . & . & . \\
\hline G rhiz & Circumbor. & Adoxa moschatellina & . & . & . & . & . & . & . & . & . & . & . & . \\
\hline G bulb & C Europ. & Corydalis pumila & . & . & . & . & . & . & . & . & . & . & . & . \\
\hline H scap & Europ.-Caucas. & Lamium galeobdolon subsp. montanum & . & . & . & . & . & . & + & . & 1 & . & . & . \\
\hline H scap & Eurosib. & Thalictrum aquilegifolium subsp. aquilegifolium & . & . & . & . & . & . & . & . & . & . & . & . \\
\hline G bulb & Europ.-Caucas. & Corydalis cava subsp. cava & . & . & . & . & . & . & . & . & . & . & . & . \\
\hline \multicolumn{15}{|c|}{ Transgressive species from the order Quercetalia pubescenti-petraeae } \\
\hline G rhiz & Circumbor. & Hepatica nobilis & + & 1 & + & 1 & + & 1 & + & + & + & + & + & 1 \\
\hline P scap & S Europ.-Sudsib. & Fraxinus ornus subsp. ornus & 1 & . & 1 & 1 & + & . & . & . & . & + & 1 & 1 \\
\hline$P$ caesp & C Europ. & Crataegus laevigata & + & + & + & 1 & . & . & + & 1 & + & + & . & . \\
\hline G rhiz & Europ.-Caucas. & Galium odoratum & . & . & . & . & + & . & + & . & . & $\mathrm{r}$ & . & . \\
\hline$P$ caesp & S Europ.-Sudsib. & Laburnum anagyroides subsp. anagyroides & + & + & + & + & . & + & . & + & . & 1 & . & . \\
\hline P scap & SE Europ. & Ostrya carpinifolia & . & . & . & . & . & . & + & . & . & + & + & 2 \\
\hline$P$ caesp & Medit. mont. & Euonymus latifolius & + & . & . & . & . & . & . & . & + & . & . & . \\
\hline G bulb & Orof. C Europ. & Lilium bulbiferum subsp. croceum & . & $\mathrm{r}$ & + & + & . & . & . & + & . & $\mathrm{r}$ & $\mathrm{r}$ & + \\
\hline H scap & C Europ. & Melittis melissophyllum subsp. melissophyllum & + & $\mathrm{r}$ & + & . & . & . & . & . & . & . & . & . \\
\hline G rhiz & Eurasiat. & Cephalanthera longifolia & $\mathrm{r}$ & . & + & . & . & . & . & . & $\mathrm{r}$ & . & . & . \\
\hline G rhiz & Endem. & Helleborus bocconei subsp. bocconei & . & $\mathrm{r}$ & . & . & . & . & . & . & . & $\mathrm{r}$ & . & . \\
\hline$P$ caesp & C Europ. & Viburnum lantana & $\mathrm{r}$ & . & . & + & + & + & . & . & . & . & . & . \\
\hline$P$ caesp & Europ.-Caucas. & Lonicera xylosteum & + & . & . & + & + & . & . & + & + & . & . & . \\
\hline
\end{tabular}




\begin{tabular}{|c|c|c|c|c|c|c|c|c|c|c|c|c|c|c|c|c|c|c|c|c|c|c|c|c|c|c|}
\hline 13 & 14 & 15 & 16 & 17 & 18 & 19 & 20 & 21 & 22 & 23 & 24 & 25 & 26 & 27 & 28 & 29 & 30 & 31 & 32 & 33 & 34 & 35 & 36 & 37 & Pres & Freq \\
\hline+ & . & . & . & . & . & + & + & . & + & + & + & . & . & . & + & 1 & + & 1 & . & + & . & . & . & . & 13 & 35 \\
\hline . & . & 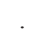 & . & . & . & + & 1 & + & 1 & + & + & + & + & . & + & . & . & . & . & + & . & 2 & . & + & 13 & 35 \\
\hline . & . & . & . & + & . & . & . & . & + & 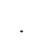 & . & . & + & + & + & + & + & + & + & . & . & . & + & . & 13 & 35 \\
\hline+ & . & 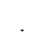 & . & . & . & . & . & . & . & 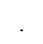 & . & $\mathrm{r}$ & . & . & + & + & + & + & + & . & . & . & . & . & 12 & 32 \\
\hline . & . & + & + & + & + & + & 1 & 1 & + & + & . & + & . & . & . & . & . & . & . & + & . & . & . & . & 11 & 30 \\
\hline . & . & 1 & + & . & . & . & . & 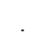 & . & 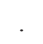 & . & + & . & . & . & + & + & . & + & . & + & . & . & . & 11 & 30 \\
\hline . & . & 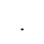 & . & 1 & . & 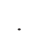 & . & 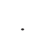 & + & 3 & 3 & . & 1 & 1 & . & . & . & . & . & 1 & 1 & 1 & . & . & 10 & 27 \\
\hline . & . & . & + & . & . & . & . & . & + & 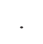 & . & . & + & . & . & + & + & + & . & . & . & . & + & . & 10 & 27 \\
\hline . & . & 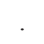 & . & . & + & 1 & . & 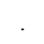 & . & 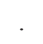 & . & . & . & . & 2 & + & + & + & + & . & . & 1 & 1 & 2 & 10 & 27 \\
\hline . & . & + & + & + & . & + & + & + & + & . & . & . & + & . & . & . & . & . & . & . & . & . & . & . & 9 & 24 \\
\hline . & . & . & . & . & . & . & . & 2 & . & 1 & . & + & + & 2 & + & . & . & + & + & . & . & . & . & . & 9 & 24 \\
\hline . & + & 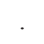 & . & . & . & 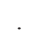 & . & . & . & 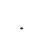 & . & + & . & . & . & . & . & . & . & . & . & + & . & . & 8 & 22 \\
\hline . & . & 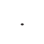 & . & . & . & . & . & . & . & . & . & 1 & + & + & + & + & + & + & + & . & . & . & . & . & 8 & 22 \\
\hline . & . & 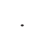 & . & . & . & . & + & . & . & . & . & . & . & . & . & . & . & . & . & . & . & . & + & 1 & 8 & 22 \\
\hline . & . & . & + & . & . & + & + & + & . & . & + & + & + & . & . & 1 & . & . & . & . & . & . & . & . & 8 & 22 \\
\hline . & . & + & + & + & . & + & + & 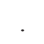 & . & 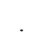 & . & . & . & . & . & . & + & . & . & . & . & + & . & . & 8 & 22 \\
\hline . & 1 & + & 1 & . & . & 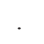 & . & 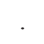 & . & . & . & . & . & . & . & . & . & . & . & . & . & . & . & . & 6 & 16 \\
\hline . & . & 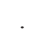 & . & . & . & . & . & + & . & $\mathrm{r}$ & . & . & . & . & + & . & . & . & . & + & . & + & . & . & 6 & 16 \\
\hline . & . & 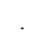 & + & . & . & 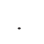 & . & 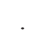 & . & $\mathrm{r}$ & . & . & . & . & + & . & + & . & . & . & . & . & . & . & 6 & 16 \\
\hline+ & . & 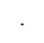 & . & . & . & . & . & 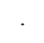 & . & . & . & . & . & . & . & . & . & . & + & . & . & . & . & . & 5 & 14 \\
\hline . & . & 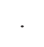 & . & . & . & . & . & 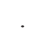 & . & $\mathrm{r}$ & . & . & . & . & + & . & + & + & . & . & . & . & . & . & 5 & 14 \\
\hline . & . & 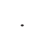 & . & . & + & 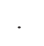 & . & . & . & . & . & . & $\mathrm{r}$ & . & . & . & . & . & . & . & . & . & . & . & 5 & 14 \\
\hline . & . & 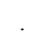 & . & . & . & . & . & + & . & $\mathrm{r}$ & . & 1 & 1 & 1 & . & . & . & . & . & . & . & . & . & . & 5 & 14 \\
\hline . & . & 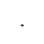 & . & . & . & 1 & . & 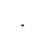 & . & . & . & . & . & . & + & + & + & . & . & . & . & . & . & . & 4 & 11 \\
\hline . & . & 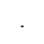 & . & . & . & + & + & 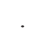 & + & . & + & . & . & . & . & . & . & . & . & . & . & . & . & . & 4 & 11 \\
\hline . & . & 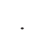 & . & . & . & . & . & . & . & . & . & . & . & . & . & . & . & . & . & . & . & . & . & . & 4 & 11 \\
\hline . & . & 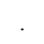 & . & . & . & . & 1 & 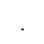 & . & 2 & 1 & . & . & . & . & . & . & . & . & . & . & . & . & . & 3 & 8 \\
\hline . & . & . & . & . & . & . & . & . & . & $\mathrm{r}$ & . & . & . & . & . & . & . & . & . & . & . & . & + & . & 3 & 8 \\
\hline . & . & 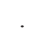 & . & . & . & . & . & . & . & 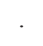 & . & . & . & . & + & . & . & . & . & . & . & . & + & + & 3 & 8 \\
\hline . & . & 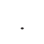 & . & + & . & . & . & 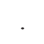 & . & . & . & . & + & . & . & . & . & . & . & + & . & . & . & . & 3 & 8 \\
\hline . & . & . & . & . & . & . & . & 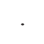 & . & . & . & . & . & . & + & + & + & . & . & . & . & . & . & . & 3 & 8 \\
\hline . & . & 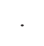 & . & . & . & . & . & 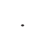 & . & 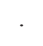 & . & . & . & . & . & . & . & . & . & . & . & . & . & . & 2 & 5 \\
\hline+ & . & 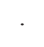 & . & . & . & . & . & 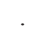 & . & . & . & . & . & . & . & . & . & . & . & . & . & . & + & . & 2 & 5 \\
\hline . & . & 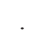 & + & + & . & . & . & 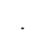 & . & . & . & . & . & . & . & . & . & . & . & . & . & . & . & . & 2 & 5 \\
\hline . & . & 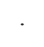 & . & . & . & . & . & 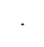 & . & . & . & . & . & . & . & . & . & . & . & . & . & . & . & . & 2 & 5 \\
\hline . & . & 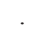 & . & . & . & . & . & . & . & . & . & . & . & . & . & . & . & . & . & . & . & + & + & . & 2 & 5 \\
\hline . & . & 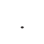 & . & . & . & . & . & 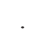 & . & 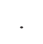 & . & + & + & . & . & . & . & . & . & . & . & . & . & . & 2 & 5 \\
\hline . & . & 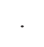 & . & . & . & . & . & 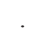 & . & . & . & . & . & . & . & . & . & . & . & . & . & . & . & . & 2 & 5 \\
\hline+ & . & . & + & . & . & . & . & 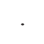 & . & . & . & . & . & . & . & . & . & . & . & . & . & . & . & . & 2 & 5 \\
\hline . & $\cdot$ & . & . & . & . &. & . & . & $\cdot$ & . & $\cdot$ & . & . & . & . & . & . & . & . & . & . & . & + & + & 2 & 5 \\
\hline
\end{tabular}

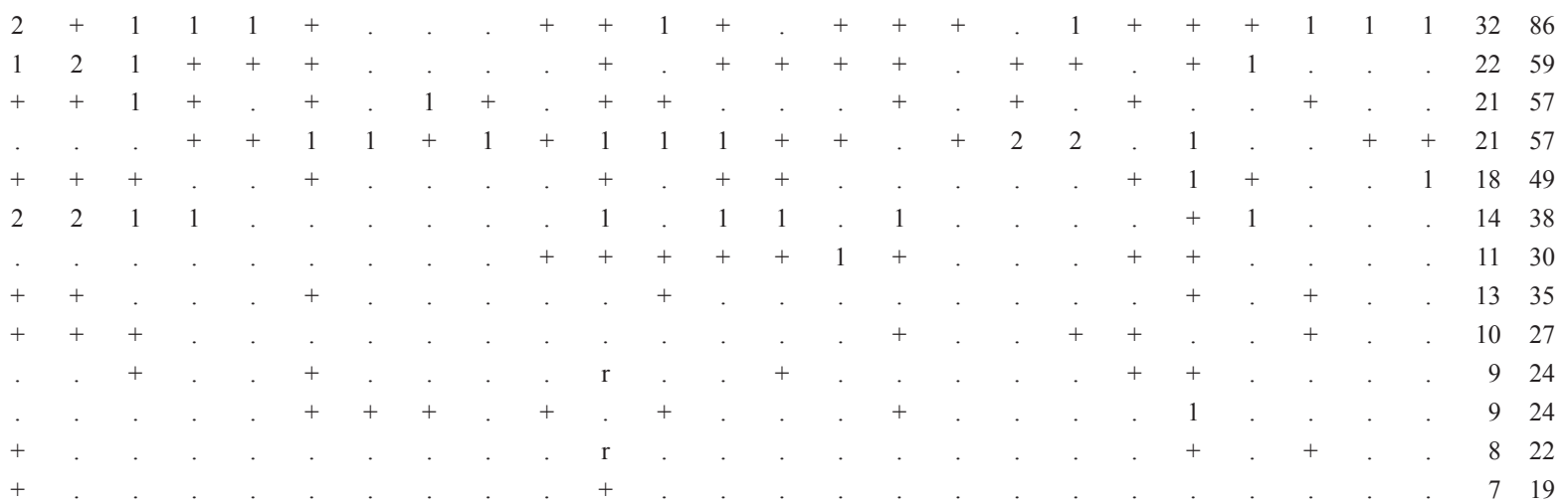




\begin{tabular}{|c|c|c|c|c|c|c|c|c|c|c|c|c|c|c|}
\hline & & Relevé number & 1 & 2 & $3 *$ & 4 & 5 & 6 & 7 & 8 & 9 & 10 & 11 & 12 \\
\hline G rhiz & & Epipactis helleborine (s.1.) & . & . & . & . & . & . & . & . & . & . & . & . \\
\hline NP & & Emerus majus (s.1.) & + & . & . & . & $\mathrm{r}$ & + & . & . & . & . & . & + \\
\hline$P$ caesp & S Europ.-Sudsib. & Cornus mas & + & + & 1 & . & $\mathrm{r}$ & . & . & + & . & . & . & . \\
\hline Ch suffr & Subatl. & Helleborus foetidus subsp. foetidus & . & . & . & . & . & . & . & . & . & . & . & . \\
\hline P scap & N Eurimedit. & Quercus cerris & . & 1 & 1 & 1 & . & . & . & . & . & . & . & 1 \\
\hline H scap & Eurasiat. & Campanula persicifolia subsp. persicifolia & . & . & + & . & . & . & . & + & + & $\mathrm{r}$ & $\mathrm{r}$ & . \\
\hline$P$ caesp & S Europ.-Sudsib. & Euonymus verrucosus & . & $\mathrm{r}$ & + & . & . & . & . & . & . & . & . & . \\
\hline$P$ caesp & Paleotemp. & Sorbus torminalis & + & + & + & . & . & . & . & . & . & + & . & . \\
\hline $\mathrm{H}$ ros & Paleotrop. & Polypodium interjectum & . & $\mathrm{r}$ & . & . & . & + & . & . & . & . & . & . \\
\hline $\mathrm{H}$ ros & Eurimedit. & Silene italica subsp. italica & . & . & . & . & . & . & . & . & . & . & . & . \\
\hline H caesp & Euromedit. & Poa sylvicola & . & . & . & . & . & . & . & . & . & . & . & . \\
\hline \multirow[t]{2}{*}{$\mathrm{H}$ ros } & Circumbor. & Polypodium vulgare & . & . & . & . & . & . & . & . & . & . & . & . \\
\hline & & Charact. species of the class Querco-Fagetea & & & & & & & & & & & & \\
\hline G bulb & Europ.-Caucas. & Galanthus nivalis subsp. nivalis & + & + & 1 & + & + & + & + & + & + & . & + & + \\
\hline H caesp & Orof. SE Europ. & Luzula sylvatica subsp. sylvatica & + & 1 & + & . & $\mathrm{r}$ & + & + & 1 & 1 & + & 1 & . \\
\hline P lian & Eurimedit. & Hedera helix subsp. helix & + & + & . & . & . & . & 1 & 1 & + & . & + & 1 \\
\hline NP & N Eurimedit. & Rubus hirtus & + & . & . & . & . & . & 1 & . & + & + & + & . \\
\hline G rhiz & Eurimedit. & Cephalanthera damasonium & . & $\mathrm{r}$ & + & . & 1 & . & . & . & . & . & . & + \\
\hline H rept & Eurosib. & Fragaria vesca subsp. vesca & . & $\mathrm{r}$ & . & . & . & . & $\mathrm{r}$ & . & $\mathrm{r}$ & + & + & + \\
\hline $\mathrm{H}$ caesp & Paleotemp. & Brachypodium sylvaticum subsp. sylvaticum & + & . & . & + & . & . & . & . & + & $\mathrm{r}$ & $\mathrm{r}$ & . \\
\hline P scap & Europ.-Caucas. & Acer campestre & . & $\mathrm{r}$ & . & 1 & + & . & . & . & + & + & + & . \\
\hline $\mathrm{H}$ ros & Europ.-Caucas. & Primula vulgaris subsp. vulgaris & + & + & . & . & . & . & + & + & + & . & . & . \\
\hline $\mathrm{H}$ ros & NE Stenomedit. & Aremonia agrimonoides subsp. agrimonoides & . & . & . & . & $\mathrm{r}$ & . & . & $\mathrm{r}$ & . & + & $\mathrm{r}$ & + \\
\hline H rept & Europ.-Caucas. & Ajuga reptans & . & . & + & . & . & . & + & + & . & . & . & + \\
\hline$P$ caesp & Europ.-Caucas. & Corylus avellana & 1 & . & . & . & . & . & . & + & + & . & . & + \\
\hline G rad & Eurimedit. & Tamus communis & + & . & + & . & . & . & $\mathrm{r}$ & . & . & . & . & . \\
\hline H scap & Circumbor. & Solidago virgaurea subsp. virgaurea & . & . & . & . & . & . & . & $\mathrm{r}$ & $\mathrm{r}$ & . & . & . \\
\hline T scap & N Medit. & Cardamine graeca & . & . & . & . & . & . & . & . & . & . & . & . \\
\hline P lian & Europ.-Caucas. & Clematis vitalba & . & . & . & . & . & . & . & . & . & + & . & . \\
\hline H scap & Europ.-Caucas. & Hieracium racemosum & . & + & . & . & . & . & . & . & . & . & . & . \\
\hline P lian & S Europ.-Sudsib. & Lonicera caprifolium & $\mathrm{r}$ & . & . & . & . & . & . & . & . & . & . & . \\
\hline G rhiz & Eurimedit. & Ruscus aculeatus & + & . & . & . & . & . & . & . & . & . & . & . \\
\hline H scap & Eurasiat. & Aquilegia vulgaris auct. fl. ital. & . & . & + & . & . & . & . & . & . & . & . & . \\
\hline \multirow[t]{2}{*}{ H scap } & Circumbor. & Geum urbanum & . & . & . & . & . & . & . & . & . & . & . & . \\
\hline & & Accompanying taxa & & & & & & & & & & & & \\
\hline H scap & Eurasiat. & Cruciata glabra subsp. glabra & . & $\mathrm{r}$ & + & + & $\mathrm{r}$ & . & . & + & + & . & $\mathrm{r}$ & + \\
\hline T scap & Eurasiat. & Moehringia trinervia & . & . & . & . & . & . & . & . & . & . & . & . \\
\hline$P$ caesp & Circumbor. & Juniperus communis subsp. communis & . & + & . & . & $\mathrm{r}$ & + & . & . & . & . & . & + \\
\hline G rhiz & Eurosib. & Aegopodium podagraria & . & . & . & . & . & . & . & $\mathrm{r}$ & . & . & . & . \\
\hline G rhiz & Cosmopol. & Pteridium aquilinum subsp. aquilinum & . & . & . & . & . & . & 1 & . & . & . & . & . \\
\hline H bienn & S Europ.-Sudsib. & Arabis turrita & . & . & $\mathrm{r}$ & . & . & . & . & . & . & . & . & . \\
\hline P caesp & Paleotemp. & Crataegus monogyna & . & . & . & . & . & . & . & . & . & . & + & . \\
\hline H scap & Europ. & Laserpitium latifolium & . & . & . & . & . & . & . & . & . & + & . & . \\
\hline \multirow[t]{2}{*}{$\mathrm{H}$ ros } & Cosmop. temp. & Asplenium trichomanes subsp. quadrivalens & . & . & . & . & . & . & . & . & . & . & . & . \\
\hline & & Sporadic taxa & 2 & 0 & 1 & 0 & 1 & 1 & 0 & 0 & 1 & 0 & 1 & 0 \\
\hline
\end{tabular}




\begin{tabular}{|c|c|c|c|c|c|c|c|c|c|c|c|c|c|c|c|c|c|c|c|c|c|c|c|c|c|c|}
\hline 13 & 14 & 15 & 16 & 17 & 18 & 19 & 20 & 21 & 22 & 23 & 24 & 25 & 26 & 27 & 28 & 29 & 30 & 31 & 32 & 33 & 34 & 35 & 36 & 37 & Pres & Freq \\
\hline . & . & . & . & . & + & . & . & . & . & . & . & $\cdot$ & . & $\cdot$ & . & + & + & . & + & . & + & + & + & . & 7 & 19 \\
\hline+ & 1 & . & . & . & . & . & 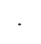 & . & . & . & . & . & . & . & . & . & . & 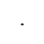 & . & 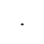 & . & 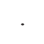 & . & . & 6 & 16 \\
\hline . & . & . & . & . & . & . & . & . & . & + & . & . & . & . & . & . & . & . & . & . & . & 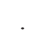 & . & . & 6 & 16 \\
\hline . & . & + & + & + & . & . & . & . & . & . & . & . & . & . & . & . & . & + & + & + & . & 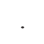 & . & . & 6 & 16 \\
\hline 1 & 2 & . & . & . & . & . & . & . & . & . & . & . & . & . & . & . & . & . & . & . & . & 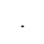 & . & . & 6 & 16 \\
\hline . & . & . & . & . & . & . & . & . & . & . & . & . & . & . & . & . & . & . & . & 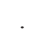 & . & 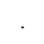 & . & . & 5 & 14 \\
\hline 1 & . & . & . & . & . & . & . & . & . & . & . & . & . & . & . & . & . & . & . & + & . & + & . & . & 5 & 14 \\
\hline . & . & . & . & . & . & . & . & . & . & . & . & . & . &. & . & . & . & . & . & . & . & 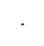 & . & . & 4 & 11 \\
\hline . & $\cdot$ & . & . & . & . & . & . & . & . & $\mathrm{r}$ & $\cdot$ & . & . & . & . & . & . & . & . & . & . & . & . & . & 3 & 8 \\
\hline . & . & 1 & . & + & + & . & . & . & . & . & . & . & . & . & . & . & . & . & . & . & . & . & . & . & 3 & 8 \\
\hline . & . & . & . & . & . & . & . & . & . & . & . & . & . & 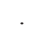 & . & . & . &. & . & . & . & . & + & + & 2 & 5 \\
\hline . & . & . & . & . & . & . & . & . & . & . & . & . & . & . & . & . & . & . & + & . & + & . & . & . & 2 & 5 \\
\hline
\end{tabular}
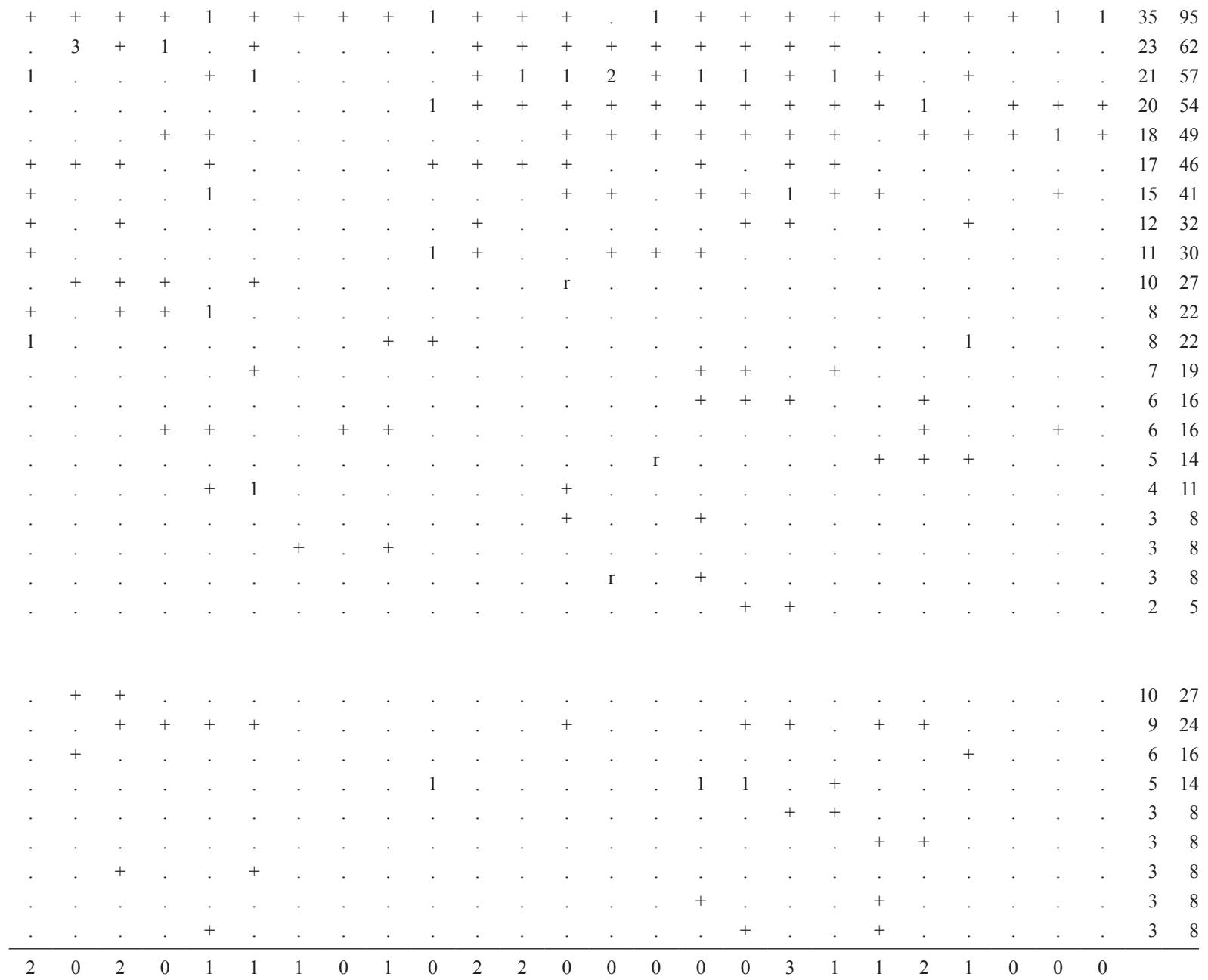
Table 6: Synoptical table of Fagus sylvatica subsp. sylvatica communities of lower supratemperate bioclimatic belt: Lv-Fs $1 \mathrm{v}$ - Lathyro veneti-Fagetum sylvaticae lathyretosum veneti Biondi, Casavecchia, Pinzi, Allegrezza et Baldoni 2002; Lv-Fs h m - Lathyro veneti-Fagetum sylvaticae Biondi, Casavecchia, Pinzi, Allegrezza et Baldoni 2002 hieracietosum murori subass. nova; Hr-Fs 1 s - Hieracio racemosi-Fagetum sylvaticae luzuletosum sylvaticae Allegrezza 2003.

Tabela 6: Sinoptična tabela združb vrste Fagus sylvatica subsp. sylvatica spodnjega supratemperatnega bioklimatskega pasu: Lv-Fs $1 \mathrm{v}$ - Lathyro veneti-Fagetum sylvaticae lathyretosum veneti Biondi, Casavecchia, Pinzi, Allegrezza et Baldoni 2002; Lv-Fs h m - Lathyro veneti-Fagetum sylvaticae Biondi, Casavecchia, Pinzi, Allegrezza et Baldoni 2002 hieracietosum murori subass. nova; Hr-Fs 1 s - Hieracio racemosi-Fagetum sylvaticae luzuletosum sylvaticae Allegrezza 2003.

\begin{tabular}{|c|c|c|c|c|c|}
\hline & & & Lv-Fs lv & Lv-Fs h m & Hr-Fs $1 \mathrm{~s}$ \\
\hline & & \multicolumn{4}{|c|}{$\begin{array}{l}\text { Characteristic species of the association Lathyro veneti-Fagetum sylvaticae and differential species of the } \\
\text { subassociation lathyretosum veneti }\end{array}$} \\
\hline G rhiz & S Europ.-Sudsib. & Lathyrus venetus & $\mathrm{V}$ & $\mathrm{V}$ & II \\
\hline Ch suffr & Europ.-Caucas. & Euphorbia amygdaloides subsp. amygdaloides & IV & I & II \\
\hline$P$ caesp & Paleotemp. & Sorbus aria subsp. aria & II & III & I \\
\hline H ros & Eurimedit. & Viola alba subsp. dehnhardtii & II & II & I \\
\hline G bulb & Europ.-Caucas. & Galanthus nivalis subsp. nivalis & $\mathrm{V}$ & $\mathrm{V}$ & . \\
\hline G rhiz & Europ.-Caucas. & Galium odoratum & $\mathrm{V}$ & II & . \\
\hline G bulb & Europ.-Caucas. & Scilla bifolia & III & III & . \\
\hline H scap & Europ.-Caucas. & Prenanthes purpurea & III & I & . \\
\hline G rhiz & SE Europ. & Cardamine enneaphyllos & I & I & . \\
\hline \multirow[t]{2}{*}{ G rhiz } & Orof. SE Europ. & Cardamine kitaibelii & II & . & . \\
\hline & & Acidophilous species & & & \\
\hline H caesp & Orof. SE Europ. & Luzula sylvatica subsp. sylvatica & IV & IV & IV \\
\hline H scap & Eurosib. & Hieracium murorum & I & III & $\mathrm{V}$ \\
\hline NP & S Medit.-Subatl. & Rosa arvensis & I & $\mathrm{V}$ & I \\
\hline H caesp & Eurimedit. & Luzula forsteri & I & II & IV \\
\hline G rhiz & Cosmopol. & Pteridium aquilinum subsp. aquilinum & I & I & $\mathrm{V}$ \\
\hline H scap & Europ.-Caucas. & Hieracium racemosum & I & I & $\mathrm{V}$ \\
\hline G rhiz & Eurasiat. & Cephalanthera rubra & I & II & I \\
\hline H caesp & Europ.-E Asiat. & Carex sylvatica subsp. sylvatica & I & I & . \\
\hline P scap & N Eurimedit. & Quercus cerris & . & II & II \\
\hline NP & W Eurimedit. & Hypericum androsaemum & . & I & I \\
\hline H ros & Eurimedit. & Potentilla micrantha & . & II & . \\
\hline \multirow[t]{2}{*}{ P caesp } & Stenomedit. & Erica arborea & . & . & III \\
\hline & & Charact. and diff. Species of the alliance Geranio & cae & & \\
\hline$P$ caesp & Subatlant. & Daphne laureola & $\mathrm{V}$ & IV & III \\
\hline P scap & SE Europ. & Acer opalus subsp. obtusatum & IV & $\mathrm{V}$ & II \\
\hline G bulb & S Europ. & Cyclamen hederifolium subsp. hederifolium & III & IV & II \\
\hline H scap & Europ.-Caucas. & Ranunculus lanuginosus & I & I & I \\
\hline H scap & Endem. & Pulmonaria apennina & II & II & . \\
\hline G rhiz & Eurimedit. & Ruscus hypoglossum & II & I & . \\
\hline \multirow[t]{2}{*}{ G rhiz } & Europ.-Caucas. & Anemone ranunculoides & I & I & . \\
\hline & & Charact. species of the order Fagetalia sylvaticae & & & \\
\hline P scap & C Europ. & Fagus sylvatica subsp. sylvatica & $\mathrm{V}$ & $\mathrm{V}$ & $\mathrm{V}$ \\
\hline H scap & Eurosib. & Viola reichenbachiana & $\mathrm{V}$ & $\mathrm{V}$ & V \\
\hline H scap & Paleotemp. & Sanicula europaea & V & V & II \\
\hline $\mathrm{H}$ caesp & Paleotemp. & Melica uniflora & $\mathrm{V}$ & IV & $\mathrm{V}$ \\
\hline H scap & Europ.-Caucas. & Lactuca muralis & $\mathrm{V}$ & III & V \\
\hline $\mathrm{H}$ caesp & Europ.-Caucas. & Festuca heterophylla & IV & $\mathrm{V}$ & V \\
\hline P scap & Paleotemp. & Taxus baccata & III & I & I \\
\hline
\end{tabular}




\begin{tabular}{|c|c|c|c|c|c|}
\hline & & & Lv-Fs lv & Lv-Fs h m & Hr-Fs $1 \mathrm{~s}$ \\
\hline G rhiz & C Europ. & Euphorbia dulcis & II & III & $\mathrm{I}$ \\
\hline G bulb & Paleotemp. & Dactylorhiza maculata subsp. fuchsii & II & II & III \\
\hline P caesp & Eurimedit. & Ilex aquifolium & II & I & III \\
\hline H scap & Circumbor. & Solidago virgaurea subsp. virgaurea & I & I & III \\
\hline H scap & Paleotemp. & Campanula trachelium subsp. trachelium & V & III & . \\
\hline G bulb & Eurasiat. & Lilium martagon & IV & III & . \\
\hline G rhiz & C Europ. & Cardamine bulbifera & IV & I & . \\
\hline G rhiz & Eurasiat. & Neottia nidus-avis & III & III & . \\
\hline H caesp & Circumbor. & Poa nemoralis subsp. nemoralis & II & II & . \\
\hline H scap & Orof. S Europ. & Saxifraga rotundifolia subsp. rotundifolia & II & I & . \\
\hline T scap & Subcosmop. & Geranium robertianum & II & I & . \\
\hline G bulb & Eurosib. & Gagea lutea & II & I & . \\
\hline P scap & Europ.-Caucas. & Acer pseudoplatanus & II & I & . \\
\hline G rhiz & Subcosmop. & Dryopteris filix-mas & II & I & . \\
\hline G rhiz & Circumbor. & Anemone nemorosa & I & II & . \\
\hline P scap & Pont. & Prunus avium subsp. avium & I & II & . \\
\hline H scap & Paleotemp. & Heracleum sphondylium subsp. ternatum & I & I & . \\
\hline G rhiz & C e S Europ. & Epipactis muelleri & I & I & . \\
\hline G rhiz & Europ.-Caucas. & Mercurialis perennis & I & I & . \\
\hline G rhiz & C Europ. & Arum maculatum & I & I & . \\
\hline G rhiz & Paleotemp. & Epipactis leptochila & I & I & . \\
\hline Ch rept & Circumbor. & Orthilia secunda & I & I & . \\
\hline T rept & Cosmopol. & Stellaria media subsp. media & I & . & I \\
\hline H rept & Eurasiat. & Veronica officinalis & . & I & I \\
\hline P scap & Europ.-Caucas. & Acer platanoides & III & & . \\
\hline G rhiz & Circumbor. & Polystichum setiferum & III & . & . \\
\hline H caesp & Eurasiat. & Bromus ramosus & II & . & . \\
\hline P scap & Europ.-Caucas. & Tilia platyphyllos subsp. platyphyllos & II & . & . \\
\hline Ch scap & Europ.-Caucas. & Stellaria holostea subsp. holostea & II & . & . \\
\hline G rhiz & Eurasiat. & Polygonatum multiflorum & I & . & . \\
\hline G rhiz & Orof. SE-Europ. & Doronicum columnae & I & . & . \\
\hline G rhiz & Eurasiat. & Actaea spicata & I & . & . \\
\hline G bulb & C Europ. & Corydalis pumila & I & . & . \\
\hline G bulb & Eurasiat. & Allium ursinum subsp. ursinum & I & . & . \\
\hline H scap & Orof. S Europ. & Aconitum lycoctonum & I & . & . \\
\hline H scap & Orof. Eurasiat. & Salvia glutinosa & I & . & . \\
\hline $\mathrm{H}$ ros & Circumbor. temp. & Phyllitis scolopendrium subsp. scolopendrium & I & . & . \\
\hline H caesp & Subatl. & Festuca altissima & I & . & . \\
\hline P caesp & S Europ.-Sudsib. & Staphylea pinnata & I & . & . \\
\hline H caesp & Europ.-Caucas. & Hordelymus europaeus & I & . & . \\
\hline G bulb & Eurasiat. & Allium ursinum subsp. ucrainicum & . & II & . \\
\hline P scap & Europ.-Caucas. & Carpinus betulus & . & I & . \\
\hline G rhiz & N Medit. mont. & Geranium nodosum & . & I & . \\
\hline H scap & NE Medit. mont. & Adenostyles glabra subsp. glabra & . & I & . \\
\hline P caesp & Orof. S Europ. & Laburnum alpinum & . & I & . \\
\hline H scap & Europ.-Caucas. & Lamium galeobdolon subsp. montanum & . & I & . \\
\hline H scap & Eurosib. & Thalictrum aquilegifolium i aquilegifolium & . & I & . \\
\hline P caesp & Europ. & Sorbus aucuparia subsp. aucuparia & . & I & . \\
\hline P scap & Europ.-Caucas. & Fraxinus excelsior subsp. excelsior & . & I & . \\
\hline H scap & & Stellaria nemorum (s.1.) & . & I & . \\
\hline \multirow[t]{2}{*}{ G rhiz } & Circumbor. & Corallorhiza trifida & . & I & . \\
\hline & & Transgressive species from the order Quercetalia pubescenti-petraeae & & & \\
\hline G rhiz & Circumbor. & Hepatica nobilis & IV & $\mathrm{V}$ & II \\
\hline P caesp & C Europ. & Crataegus laevigata & III & IV & II \\
\hline H caesp & Eurasiat. & Carex digitata & I & $\mathrm{V}$ & II \\
\hline G rhiz & Eurasiat. & Cephalanthera longifolia & I & II & IV \\
\hline
\end{tabular}




\begin{tabular}{|c|c|c|c|c|c|}
\hline & & & Lv-Fs lv & Lv-Fs h m & $\overline{\mathrm{Hr}-\mathrm{Fs} \mathrm{ls}}$ \\
\hline H scap & Endem. & Digitalis lutea subsp. australis & I & II & III \\
\hline$P$ caesp & Europ.-Caucas. & Lonicera xylosteum & I & II & II \\
\hline P scap & S Europ.-Sudsib. & Fraxinus ornus subsp. ornus & III & IV & . \\
\hline$P$ caesp & Medit. mont. & Euonymus latifolius & III & I & . \\
\hline P caesp & S Europ.-Sudsib. & Laburnum anagyroides subsp. anagyroides & II & III & . \\
\hline P scap & SE Europ. & Ostrya carpinifolia & II & III & . \\
\hline G rhiz & Endem. & Helleborus bocconei subsp. bocconei & II & I & . \\
\hline G bulb & Orof. C Europ. & Lilium bulbiferum subsp. croceum & I & III & . \\
\hline H scap & C Europ. & Melittis melissophyllum subsp. melissophyllum & I & II & . \\
\hline P caesp & C Europ. & Viburnum lantana & I & II & . \\
\hline$P$ caesp & S Europ.-Sudsib. & Cornus mas & I & II & . \\
\hline Ch suffr & Subatl. & Helleborus foetidus subsp. foetidus & I & I & . \\
\hline G rhiz & & Epipactis helleborine (s.1.) & I & I & . \\
\hline $\mathrm{H}$ ros & Paleotrop. & Polypodium interjectum & I & I & . \\
\hline $\mathrm{H}$ ros & Circumbor. & Polypodium vulgare & I & . & III \\
\hline NP & & Emerus majus (s.1.) & . & II & III \\
\hline $\mathrm{H}$ ros & Eurimedit. & Silene italica subsp. italica & . & I & III \\
\hline H scap & Eurasiat. & Campanula persicifolia subsp. persicifolia & . & II & . \\
\hline$P$ caesp & Paleotemp. & Sorbus torminalis & . & I & . \\
\hline$P$ caesp & S Europ.-Sudsib. & Euonymus verrucosus & . & I & . \\
\hline H caesp & Subatl. & Brachypodium rupestre & . & I & . \\
\hline$P$ caesp & Eurasiat. & Euonymus europaeus & . & I & . \\
\hline H caesp & Europ.-Caucas. & Hypericum montanum & . & I & . \\
\hline \multirow[t]{2}{*}{ H scap } & Eurasiat. & Vincetoxicum hirundinaria subsp. hirundinaria & . & I & . \\
\hline & & Charact. species of the class Querco-Fagetea & & & \\
\hline $\mathrm{P}$ lian & Eurimedit. & Hedera helix subsp. helix & IV & III & III \\
\hline NP & N Eurimedit. & Rubus hirtus & IV & II & $\mathrm{V}$ \\
\hline H rept & Eurosib. & Fragaria vesca subsp. vesca & III & III & IV \\
\hline $\mathrm{H}$ ros & Europ.-Caucas. & Primula vulgaris subsp. vulgaris & II & II & II \\
\hline P scap & Europ.-Caucas. & Acer campestre & I & III & II \\
\hline $\mathrm{G} \mathrm{rad}$ & Eurimedit. & Tamus communis & I & I & III \\
\hline $\mathrm{P}$ lian & S Europ.-Sudsib. & Lonicera caprifolium & I & I & I \\
\hline G rhiz & SE Europ. & Anemone apennina subsp. apennina & $\mathrm{V}$ & $\mathrm{V}$ & . \\
\hline H caesp & Paleotemp. & Brachypodium sylvaticum subsp. sylvaticum & III & II & . \\
\hline G rhiz & Eurimedit. & Cephalanthera damasonium & III & II & . \\
\hline $\mathrm{H}$ ros & NE Stenomedit. & Aremonia agrimonoides subsp. agrimonoides & I & III & . \\
\hline P caesp & Europ.-Caucas. & Corylus avellana & I & II & . \\
\hline T scap & N Medit. & Cardamine graeca & I & I & . \\
\hline P lian & Europ.-Caucas. & Clematis vitalba & I & I & . \\
\hline G rhiz & Eurimedit. & Ruscus aculeatus & I & I & . \\
\hline H scap & Eurasiat. & Aquilegia vulgaris auct. fl. ital. & I & I & . \\
\hline H scap & Eurasiat. & Cruciata glabra subsp. glabra & . & III & IV \\
\hline H rept & Europ.-Caucas. & Ajuga reptans & . & III & I \\
\hline G bulb & Eurosib. & Platanthera chlorantha & . & II & III \\
\hline H scap & Circumbor. & Geum urbanum & I & . & . \\
\hline G rhiz & Eurasiat. & Listera ovata & . & I & . \\
\hline$P$ caesp & SE Europ. & Quercus pubescens subsp. pubescens & . & . & III \\
\hline P scap & SE Europ. & Castanea sativa & . & . & III \\
\hline H caesp & Eurasiat. & Anthoxanthum odoratum & . & . & I \\
\hline \multirow[t]{2}{*}{ G bulb } & NW Stenomedit. & Cyclamen repandum subsp. repandum & . & . & I \\
\hline & & Accompanying taxa & & & \\
\hline T scap & Eurasiat. & Moehringia trinervia & II & I & . \\
\hline G rhiz & Eurosib. & Aegopodium podagraria & II & I & . \\
\hline H scap & Europ. & Laserpitium latifolium & I & I & . \\
\hline $\mathrm{H}$ ros & Cosmop. temp. & Asplenium trichomanes subsp. quadrivalens & I & I & . \\
\hline
\end{tabular}


Andrea Catorci et al.: Phytosociological Study of Beegh and Beegh-mixed Woods in Mt. Sibillini National Park

\begin{tabular}{|c|c|c|c|c|c|}
\hline & & & Lv-Fs lv & Lv-Fs h m & Hr-Fs $1 \mathrm{~s}$ \\
\hline H bienn & S Europ.-Sudsib. & Arabis turrita & I & I & . \\
\hline H scap & Endem. & Campanula micrantha & I & I & . \\
\hline H caesp & Cosmopol. & Cystopteris fragilis & I & I & . \\
\hline P caesp & Circumbor. & Juniperus communis subsp. communis & . & II & II \\
\hline$P$ caesp & Paleotemp. & Crataegus monogyna & . & I & II \\
\hline $\mathrm{H}$ ros & Cosmopol. & Asplenium adiantum-nigrum subsp. adiantum-nigrum & . & I & II \\
\hline NP & Eurasiat. & Rosa canina & . & I & II \\
\hline $\mathrm{H}$ ros & S Europ.-W Asiat. & Ceterach officinarum subsp. officinarum & I & . & . \\
\hline H caesp & Orof. S e C Europ. & Moehringia muscosa & I & . & . \\
\hline H scap & Eurasiat. & Lamium maculatum & I & . & . \\
\hline $\mathrm{H}$ ros & W Europ. (Subatl.) & Primula veris subsp. suaveolens & I & . & . \\
\hline H scap & C Europ. & Senecio ovatus subsp. alpestris & I & . & . \\
\hline T scap & Eurasiat. & Chaerophyllum temulum & I & . & . \\
\hline$P$ caesp & Europ.-Caucas. & Sambucus nigra & I & . & . \\
\hline NP & Eurosib. & Ribes alpinum & . & I & . \\
\hline H caesp & Endem. & Sesleria nitida (s.1.) & . & I & . \\
\hline$P$ caesp & Eurasiat. & Salix caprea & . & I & . \\
\hline$P$ caesp & Orof. SW Europ. & Cytisophyllum sessilifolium & . & I & . \\
\hline $\mathrm{H}$ ros & Subtrop. nesicola & Asplenium onopteris & . & I & . \\
\hline NP & Europ.-Caucas. & Ligustrum vulgare & . & I & . \\
\hline Ch suffr & Eurasiat. & Genista tinctoria & . & . & II \\
\hline
\end{tabular}




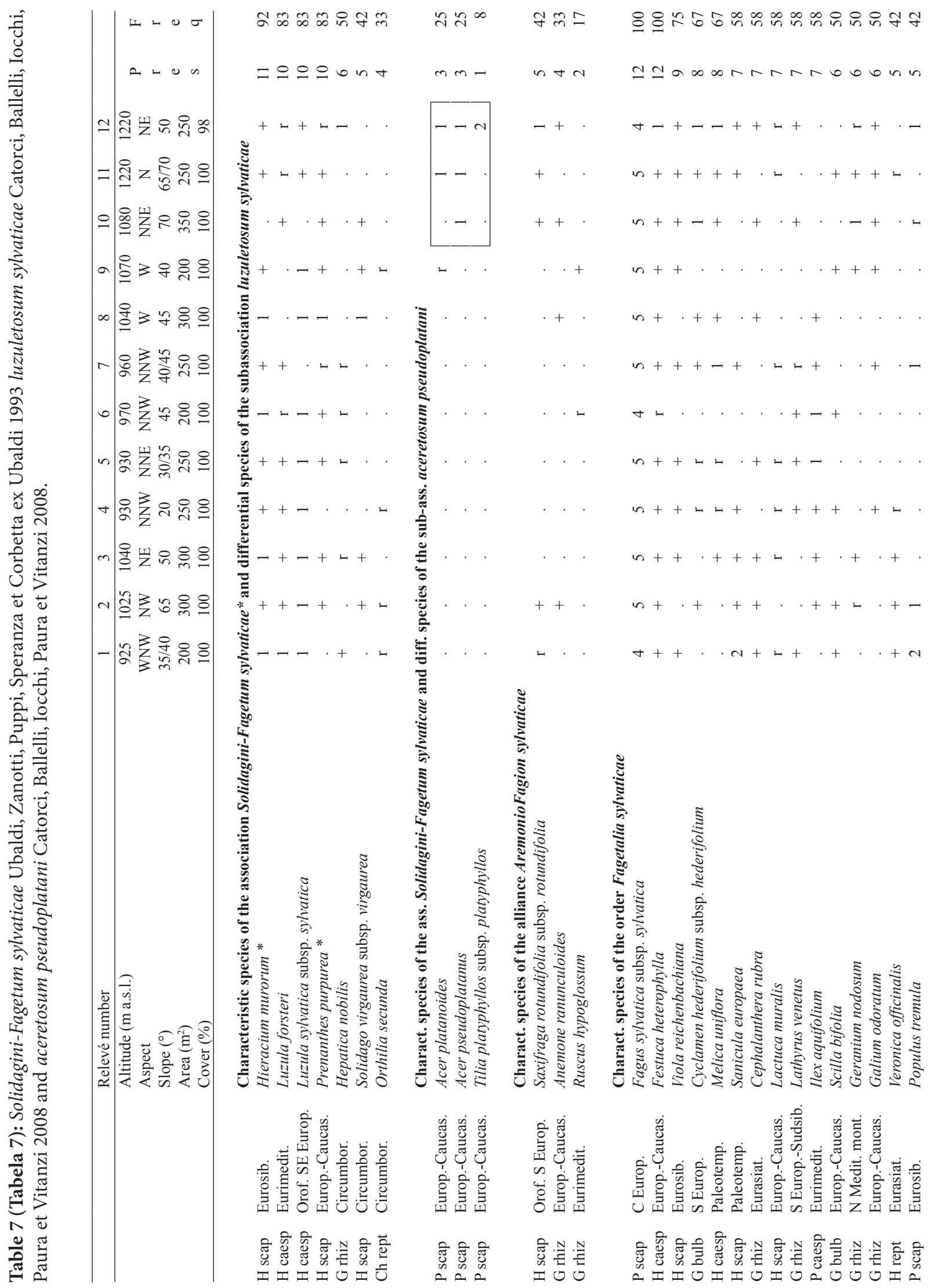




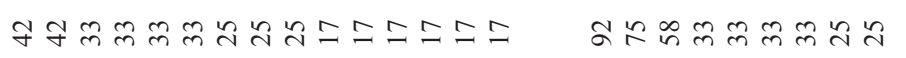

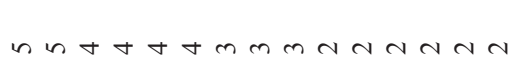
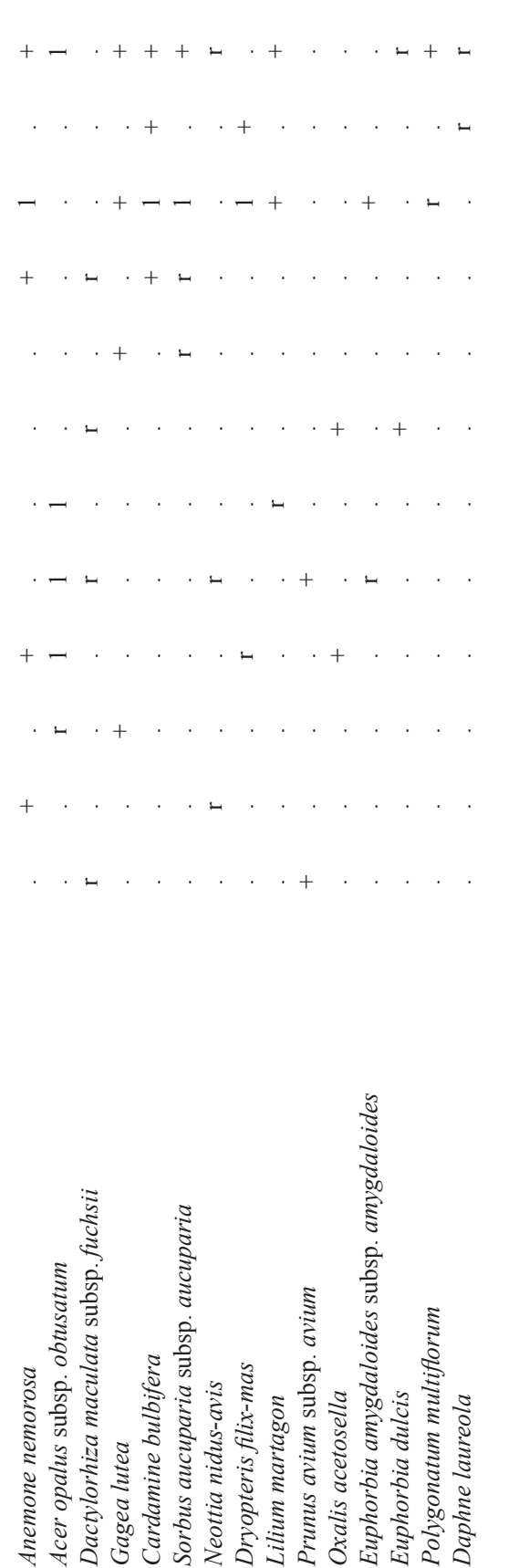

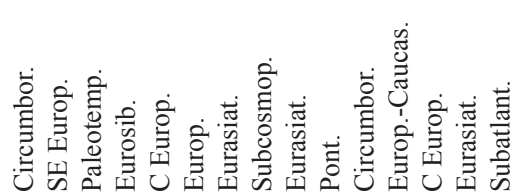

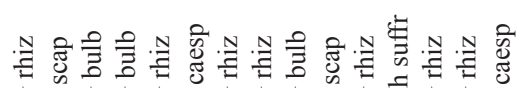

$=a r+t+t \tan$
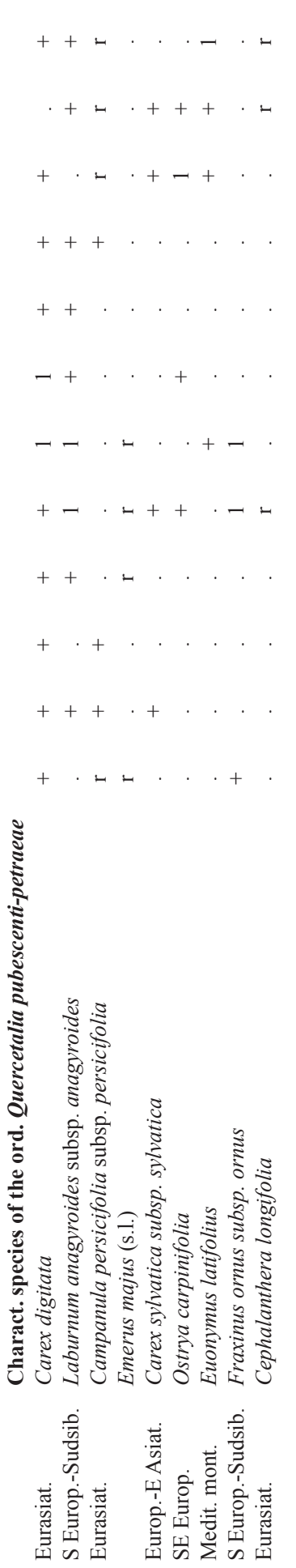

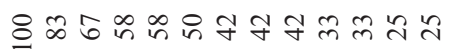

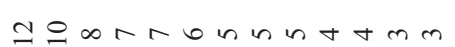
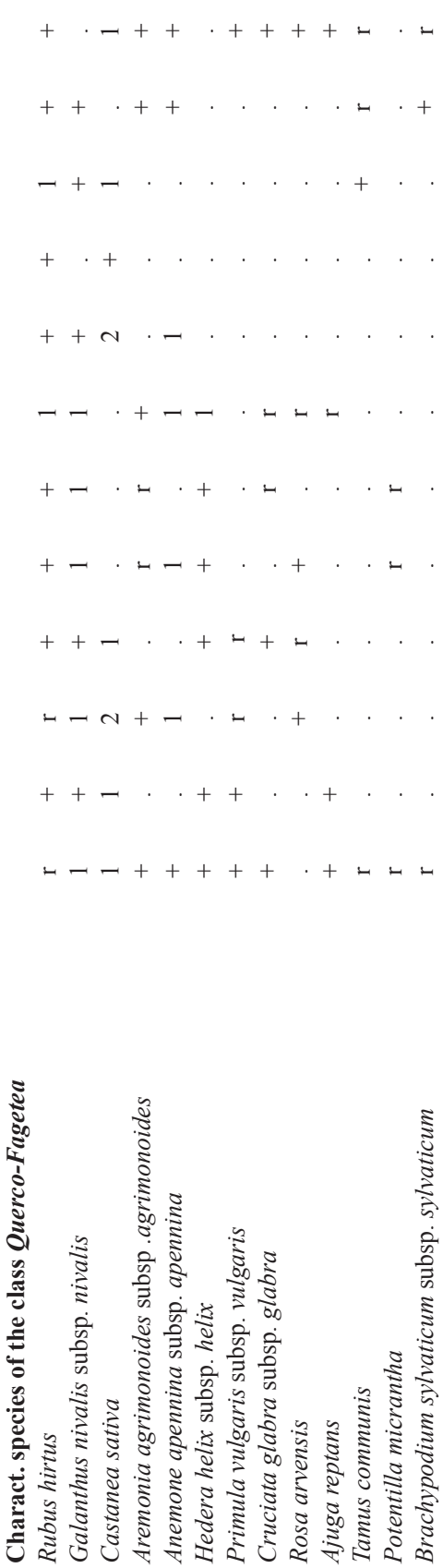

$\stackrel{\infty}{\infty} \stackrel{\infty}{i}$
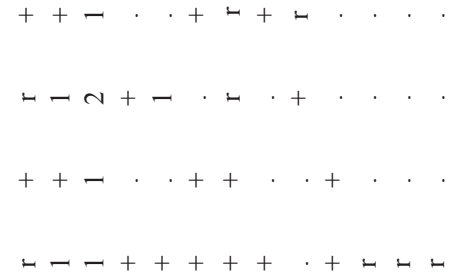

$n$

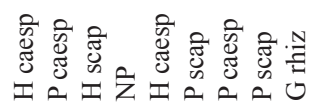

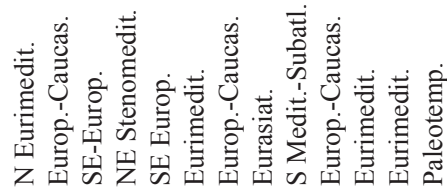

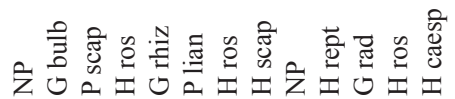


Table 8 (Tabela 8): Cardamino kitaibelii-Fagetum sylvaticae Ubaldi, Zanotti, Puppi, Speranza et Corbetta ex Ubaldi 1993 cardaminetosum kitaibelii subass. nova Sorbus aucuparia subsp. aucuparia variant and Polystichum setiferum variant.

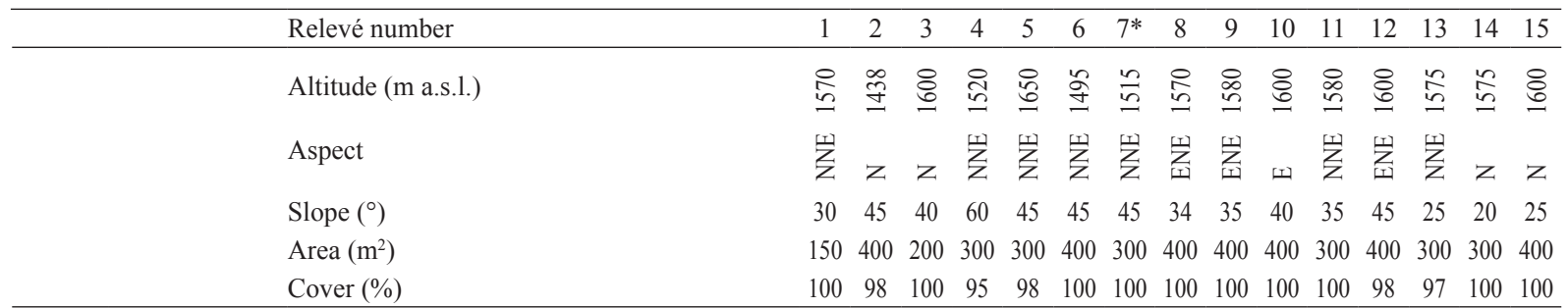

$\begin{array}{lll}\text { G rhiz } & \text { SE Europ. } & \begin{array}{l}\text { Characteristic species of the } \\ \text { Cardamine enneaphyllos }\end{array} \\ \text { G rhiz } & \text { Circumbor. } & \text { Adoxa moschatellina } \\ \text { G rhiz } & \text { Eurasiat. } & \text { Lathyrus vernus subsp. vernus } \\ \text { G rhiz } & \text { Orof. SE Europ. } & \text { Cardamine kitaibelii } \\ \text { H scap } & \text { Eurasiat. } & \text { Epilobium montanum } \\ \text { G rhiz } & \text { Eurasiat. } & \text { Polystichum aculeatum }\end{array}$

Species of the Sorbus aucuparia subsp. aucuparia variant

P caesp Paleotemp. Sorbus aria subsp. aria

P caesp Europ. Sorbus aucuparia subsp. aucuparia

P caesp Orof. S Europ. Laburnum alpinum

G rhiz Circumbor. Polystichum setiferum

P scap Europ.-Caucas. Acer pseudoplatanus

P scap Europ.-Caucas. Acer platanoides

P scap Europ.-Caucas. Fraxinus excelsior subsp. excelsior

G rhiz Orof. SE Europ. Asperula taurina subsp. taurina

Charact. species of the alliance Aremonio-Fagion sylvaticae* and diff. of the suballiance Cardamino kitaibelii-

H scap Orof. S Europ. Saxifraga rotundifolia subsp. rotundifolia*

H scap Europ.-Caucas. Ranunculus lanuginosus*

$\mathrm{H}$ ros NE Stenomedit. Aremonia agrimonoides subsp. agrimonoides*

G rhiz Europ.-Caucas. Anemone ranunculoides*

G rhiz Eurimedit. Ruscus hypoglossum*

H scap NE Medit. mont. Adenostyles glabra subsp. glabra

H scap Endem. Pulmonaria apennina

G rhiz N Medit. mont. Geranium nodosum

G bulb Paleotemp. Dactylorhiza maculata subsp. fuchsii

Charact. species of the order Fagetalia sylvaticae

\begin{tabular}{|c|c|c|c|c|c|c|c|c|c|c|c|c|c|}
\hline P scap & C Europ. & Fagus sylvatica subsp. sylvatica & 3 & 5 & 5 & 5 & 5 & 5 & 5 & 5 & 5 & & 5 \\
\hline G rhiz & Europ.-Caucas. & Galium odoratum & + & + & + & + & + & 2 & + & 1 & 1 & & 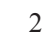 \\
\hline H scap & Eurosib. & Viola reichenbachiana & + & + & + & + & + & + & + & + & & & \\
\hline H scap & Europ.-Caucas. & Lactuca muralis & + & + & + & + & + & + & + & 1 & & & \\
\hline G rhiz & C Europ. & Cardamine bulbifera & . & + & + & + & + & 1 & + & 1 & & & \\
\hline G bulb & Europ.-Caucas. & Corydalis cava subsp. cava & + & 1 & + & + & 1 & 2 & 1 & + & & & 2 \\
\hline H scap & Europ.-Caucas. & Prenanthes purpurea & . & + & . & . & . & + & + & $\cdot$ & & & . \\
\hline G bulb & Eurasiat. & Lilium martagon & . & . & . & . & . & + & + & . & & & + \\
\hline G bulb & Europ.-Caucas. & Scilla bifolia & + & + & + & + & + & + & . & + & & & \\
\hline G rhiz & Europ.-Caucas. & Epipactis microphylla & + & + & . & . & + & + & + & . & & & + \\
\hline G rhiz & Subcosmop. & Dryopteris filix-mas & . & . & . & . & . & . & . & . & & & \\
\hline H scap & Paleotemp. & Heracleum sphondylium subsp. ternatum & . & . & . & . & . & . & . & . & & & \\
\hline H caesp & Circumbor. & Poa nemoralis subsp. nemoralis & . & & & & 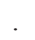 & + & 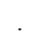 & & & & \\
\hline
\end{tabular}


\begin{tabular}{lllllllllllllllllllllllllll}
\hline 16 & 17 & 18 & 19 & 20 & 21 & 22 & 23 & 24 & 25 & 26 & 27 & 28 & 29 & 30 & 31 & 32 & 33 & 34 & 35 & 36 & 37 & 38 & 39 & 40 & 41 & 42
\end{tabular}

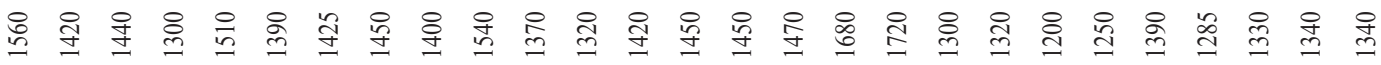

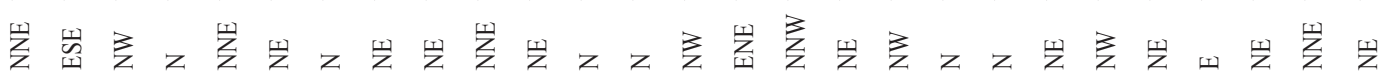
$\begin{array}{lllllllllllllllllllllllllll}55 & 15 & 20 & 30 & 35 & 25 & 25 & 25 & 25 & 30 & 20 & 15 & 40 & 45 & 55 & 30 & 30 & 40 & 20 & 20 & 45 & 25 & 30 & 20 & 45 & 25 & 25\end{array}$

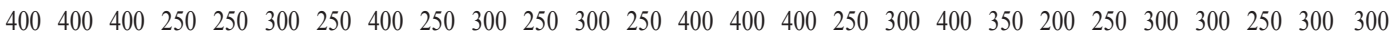
$\begin{array}{lllllllllllllllllllllllllll}98 & 100 & 100 & 100 & 97 & 95 & 98 & 97 & 98 & 97 & 100 & 100 & 100 & 100 & 100 & 100 & 100 & 100 & 100 & 100 & 100 & 100 & 100 & 100 & 100 & 100 & 100\end{array}$

\section{subassociation cardaminetosum kitaibelii}

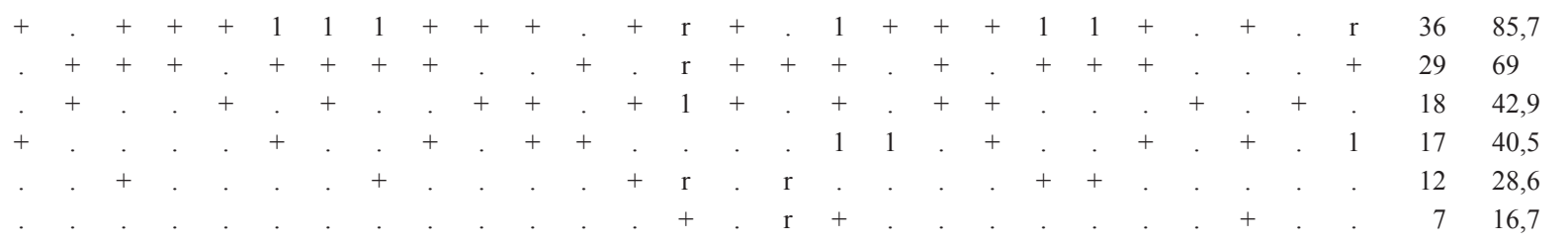

\begin{tabular}{|c|c|c|c|c|c|c|c|c|c|}
\hline+ & & + & + & 1 & 1 & 1 & + & 1 & 1 \\
\hline+ & + & 1 & + & 1 & + & 1 & 1 & 2 & + \\
\hline+ & + & . & + & . & . & + & . & . & \\
\hline
\end{tabular}

\section{-Fagenion sylvaticae}

\begin{tabular}{|ccccccc|rl}
\hline 1 & $\cdot$ & 1 & $\mathrm{r}$ & $\cdot$ & + & + & 22 & 52,4 \\
1 & 1 & 1 & 1 & 1 & $\cdot$ & $\cdot$ & 8 & 19 \\
1 & $\cdot$ & $\cdot$ & 1 & + & + & 1 & 7 & 16,7 \\
1 & $\cdot$ & 1 & $\cdot$ & 1 & + & 1 & 5 & 11,9 \\
$\cdot$ & $\cdot$ & $\mathrm{r}$ & $\cdot$ & + & $\cdot$ & + & 3 & 7,14
\end{tabular}

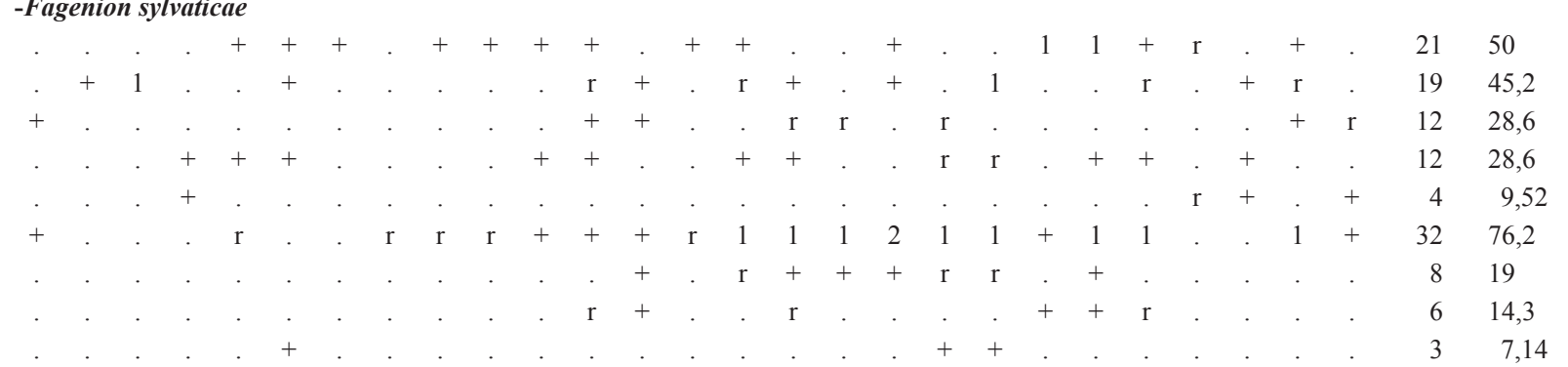

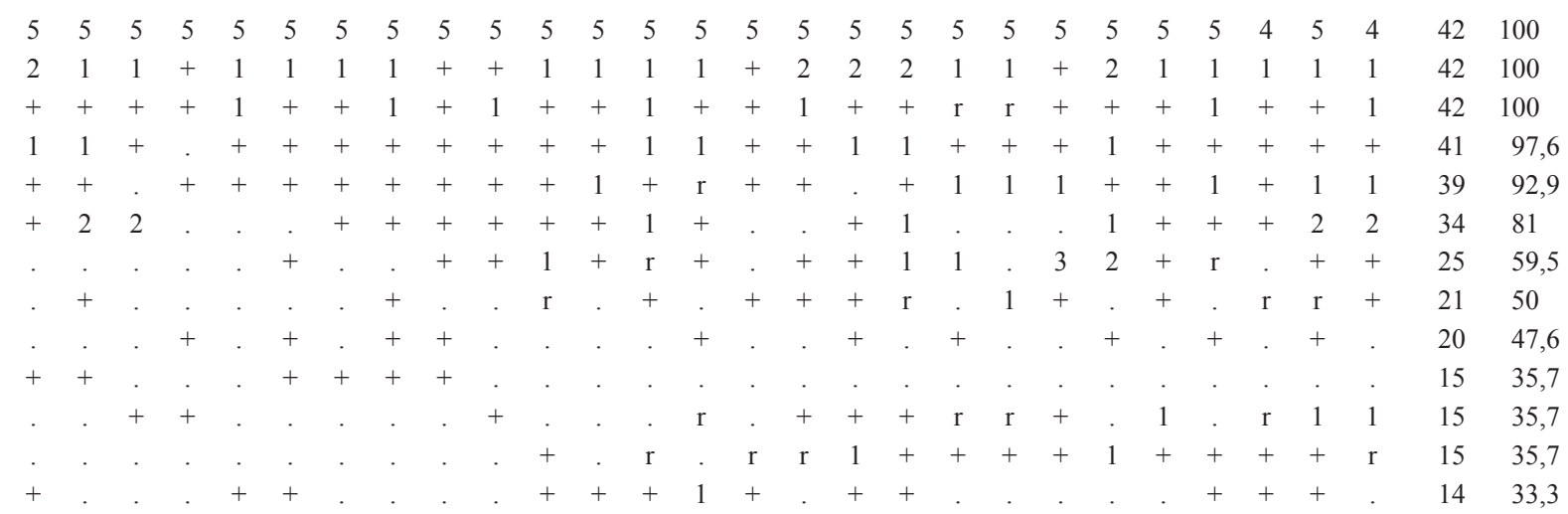




\begin{tabular}{|c|c|c|c|c|c|c|c|c|c|c|c|c|c|c|c|c|c|}
\hline & & Relevé number & 1 & 2 & 3 & 4 & 5 & 6 & 7* & 8 & 9 & 10 & 11 & 12 & 13 & 14 & 15 \\
\hline H caesp & Europ.-Caucas. & Festuca heterophylla & . & . & . & . & . & . & . & . & . & . & . & . & . & . & . \\
\hline G rhiz & Orof. SE-Europ. & Doronicum columnae & . & . & . & . & + & . & . & + & + & + & + & + & 1 & + & + \\
\hline H scap & Paleotemp. & Campanula trachelium subsp. trachelium & . & . & . & . & . & . & . & . & . & . & . & . & . & . & . \\
\hline G rhiz & C Europ. & Euphorbia dulcis & . & . & . & . & . & + & . & . & . & . & . & . & . & . & . \\
\hline G rhiz & Eurasiat. & Neottia nidus-avis & . & . & . & + & . & . & . & . & . & . & . & . & . & . & . \\
\hline T scap & Subcosmop. & Geranium robertianum & . & . & . & . & . & . & . & . & . & . & . & . & . & . & + \\
\hline G bulb & Eurosib. & Gagea lutea & . & . & . & . & + & . & . & . & + & + & . & . & . & . & + \\
\hline Ch suffr & Europ.-Caucas. & Euphorbia amygdaloides subsp. amygdaloides & . & . & . & . & . & . & . & . & . & . & . & . & . & . & . \\
\hline P scap & SE Europ. & Acer opalus subsp. obtusatum & . & + & . & + & + & . & . & . & . & . & . & . & . & . & . \\
\hline H scap & Orof. S Europ. & Aconitum lycoctonum & . & . & . & . & . & + & . & . & . & + & . & . & . & . & . \\
\hline G rhiz & C e S Europ. & Epipactis muelleri & + & . & . & . & . & . & + & + & + & . & + & + & . & . & . \\
\hline Ch scap & Europ.-Caucas. & Stellaria holostea subsp. holostea & . & . & . & . & . & . & . & . & + & + & + & + & . & . & . \\
\hline H scap & & Stellaria nemorum (s.1.) & . & . & . & . & . & . & . & . & . & . & . & . & . & . & . \\
\hline G rhiz & Eurasiat. & Polygonatum verticillatum & . & . & . & . & . & . & . & . & . & . & . & . & . & . & . \\
\hline G rhiz & Circumbor. & Polystichum lonchitis & . & . & . & . & . & + & . & . & . & . & . & . & + & . & . \\
\hline G bulb & Eurasiat. & Allium ursinum subsp. ursinum & . & . & . & . & . & . & . & . & . & . & . & . & . & . & . \\
\hline P caesp & Eurimedit. & Ilex aquifolium & . & . & . & . & . & . & . & . & . & . & . & . & . & . & . \\
\hline G rhiz & Eurasiat. & Polygonatum multiflorum & . & . & . & . & . & . & . & . & . & . & . & . & . & . & . \\
\hline G rhiz & C Europ. & Arum maculatum & . & . & . & . & . & . & . & . & . & . & . & . & . & $\cdot$ & . \\
\hline NP & Eurosib. & Ribes alpinum & . & . & . & . & . & . & . & . & . & . & . & . & . & . & . \\
\hline G bulb & C Europ. & Corydalis pumila & . & . & . & . & . & . & . & . & . & . & . & . & . & . & . \\
\hline G rhiz & Europ.-Caucas. & Mercurialis perennis & . & . & . & . & . & + & . & . & . & . & . & . & . & + & . \\
\hline H scap & Paleotemp. & Silene dioica & . & . & . & . & . & . & . & . & . & . & + & . & . & . & . \\
\hline G rhiz & Eurasiat. & Cephalanthera rubra & . & . & . & . & . & . & . & . & . & . & . & . & . & . & . \\
\hline H rept & Eurasiat. & Veronica officinalis & . & . & . & . & . & . & . & . & . & . & . & . & . & . & . \\
\hline G rhiz & Paleotemp. & Epipactis leptochila & . & . & . & . & . & . & . & . & . & . & . & . & . & . & . \\
\hline T rept & Cosmopol. & Stellaria media subsp. media & . & . & . & . & . & . & . & . & . & . & . & . & . & . & . \\
\hline P caesp & Orof. S Europ. & Lonicera alpigena subsp. alpigena & . & . & . & . & . & . & . & . & . & . & . & . & . & . & . \\
\hline P scap & Europ.-Caucas. & Ulmus glabra & . & . & . & . & . & . & . & . & . & . & . & . & . & . & . \\
\hline H caesp & Subatl. & Festuca altissima & . & . & . & . & . & . & . & . & . & . & . & . & . & . & . \\
\hline \multicolumn{18}{|c|}{ Transgressive species from the ord. Quercetalia pubescenti-petraeae } \\
\hline G rhiz & Circumbor. & Hepatica nobilis & . & + & . & . & . & + & + & + & 1 & + & + & + & + & + & . \\
\hline G rhiz & & Epipactis helleborine (s.1.) & . & . & . & . & . & . & . & . & . & . & . & . & . & . & + \\
\hline H caesp & Euromedit. & Poa sylvicola & . & . & . & . & . & + & + & + & + & . & + & + & 1 & + & + \\
\hline P caesp & Medit. mont. & Euonymus latifolius & . & . & . & . & . & . & . & . & . & . & . & . & . & . & + \\
\hline H caesp & Europ.-E Asiat. & Carex sylvatica subsp. sylvatica & . & . & . & . & . & . & . & . & . & . & . & . & . & . & . \\
\hline P caesp & S Europ.-Sudsib. & Laburnum anagyroides subsp. anagyroides & . & . & . & + & . & . & . & . & . & . & . & . & . & + & . \\
\hline G rhiz & Endem. & Helleborus bocconei subsp. bocconei & + & . & . & . & . & . & . & + & . & . & . & + & + & . & + \\
\hline H scap & Endem. & Digitalis lutea subsp. australis & . & . & . & . & . & . & . & . & . & . & . & + & . & . & + \\
\hline P caesp & C Europ. & Crataegus laevigata & . & . & . & . & . & . & . & . & . & . & . & . & . & . & . \\
\hline H scap & C Europ. & Melittis melissophyllum subsp. melissophyllum & . & . & . & . & + & . & . & . & . & . & . & . & . & . & + \\
\hline G rhiz & Eurasiat. & Cephalanthera longifolia & . & . & . & . & . & . & . & . & + & . & . & . & . & . & . \\
\hline $\mathrm{H}$ ros & Paleotrop. & Polypodium interjectum & . & . & . & . & . & . & . & . & . & . & . & . & . & . & . \\
\hline H caesp & Subatl. & Brachypodium rupestre & . & + & . & . & . & . & . & . & . & . & . & . & . & . & . \\
\hline H caesp & Eurasiat. & Carex digitata & . & . & . & . & . & . & . & . & . & . & . & . & . & . & . \\
\hline H scap & Orof. S Europ. & Calamintha nepeta subsp. nepeta & . & . & . & . & . & . & . & . & $\cdot$ & $\cdot$ & . & . & . & . & . \\
\hline H ros & Eurimedit. & Viola alba subsp. dehnhardtii & . & . & . & . & . & . & . & . & . & . & . & . & . & . & . \\
\hline G bulb & Orof. C Europ. & Lilium bulbiferum subsp. croceum & . & . & . & . & . & . & . & . & . & . & . & . & . & . & . \\
\hline \multicolumn{18}{|c|}{ Character species of the class Querco-Fagetea } \\
\hline G bulb & Europ.-Caucas. & Galanthus nivalis subsp. nivalis & + & . & + & ${ }^{\circ}$ & $\cdot$ & 1 & 1 & + & 1 & 1 & 1 & 1 & + & + & + \\
\hline H scap & Paleotemp. & Sanicula europaea & . & + & . & . & . & . & . & . & . & . & . & + & 2 & . & . \\
\hline H caesp & Paleotemp. & Melica uniflora & . & . & . & . & . & . & . & . & . & . & . & . & . & . & . \\
\hline P caesp & Subatlant. & Daphne laureola & . & . & . & . & . & . & . & . & . & . & . & . & . & . & . \\
\hline NP & N Eurimedit. & Rubus hirtus & . & . & . & $\cdot$ & . & + & + & . & . & . & . & . & . & + & . \\
\hline
\end{tabular}




\begin{tabular}{|c|c|c|c|c|c|c|c|c|c|c|c|c|c|c|c|c|c|c|c|c|c|c|c|c|c|c|c|c|}
\hline 16 & 17 & 18 & 19 & 20 & 21 & 22 & 23 & 24 & 25 & 26 & 27 & 28 & 29 & 30 & 31 & 32 & 33 & 34 & 35 & 36 & 37 & 38 & 39 & 40 & 41 & 42 & Pres & Freq \\
\hline+ & + & $\cdot$ & . & . & + & . &. & + & + & + & + & 1 & + & . & + & 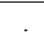 & + & + & + & . & . & . & $\mathrm{r}$ & . & 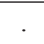 & . & 14 & 33,3 \\
\hline+ & + & . & . & . & . & . & . & . & . & . & . & . & . & . & . & . & + & . & . & . & . & . & + & . & 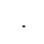 & & 13 & 31 \\
\hline r & . & . & . & + & . & . & . & + & . & + & $\mathrm{r}$ & . & + & . & . & + & $\mathrm{r}$ & . & + & + & + & + & + & $\mathrm{r}$ & 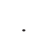 & . & 13 & 31 \\
\hline$\cdot$ & . & . & + & . & . & + & . & + & . & $\mathrm{r}$ & . & . & . & . & + & . & . & + & + & . & . & + & $\mathrm{r}$ & $\mathrm{r}$ & . & + & 12 & 28,6 \\
\hline. & . & + & + & $r$ & + & . & . & . & . & $\mathrm{r}$ & . & . & $\mathrm{r}$ & $\mathrm{r}$ & $r$ & . & $\mathrm{r}$ & . & . & . & + & . & . &. & . & . & 11 & 26,2 \\
\hline. & . & . & . & . & . & + & . & . & . & . & $\mathrm{r}$ & . & $\mathrm{r}$ & . & + & . & . & . & . & + & + & . & $\mathrm{r}$ & + & $\mathrm{r}$ & $\mathrm{r}$ & 11 & 26,2 \\
\hline. & + & . & + & + & . & . & . & + & . & . & . & . & . & + & . & + & . & . & . & . & . & . & . & . & $\cdot$ & $\cdot$ & 10 & 23,8 \\
\hline. & . & . & . & . & . & . & . & . & . & . & $\mathrm{r}$ & $\mathrm{r}$ & . & $\mathrm{r}$ & $\mathrm{r}$ & + & . & + & + & + & + & . & . & . & . & . & 0 & 21,4 \\
\hline+ & . & . & . & . & . & . & . & . & . & . & . & . & . & . & . & . & . & 1 & 1 & + & . & . & 1 & . & . & . & 8 & 19 \\
\hline$\cdot$ & . & $\cdot$ & . & $\cdot$ & . & . & . & . & . & . & . & . & . & . & . & + & + & . & $\mathrm{r}$ & + & + & + & . & . & . & . & 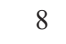 & 19 \\
\hline. & + & . & . & . & . & . & . & . & . & . & . & . & . & . & . & . & . & . & . & . & . & . & . & . & . & . & I & 16,7 \\
\hline . & . & . & . & . & . & . & . & . & . & . & . & 1 & . & . & + & . & . & . & . & . & . & . & . & . & $\mathrm{r}$ & . & 7 & 16,7 \\
\hline. & . & . & . & $\cdot$ & . & . & . & $\cdot$ & + & . & . & . & . & . & 1 & + & + & . & . & . & . & + & + & . & + & . & 7 & 16,7 \\
\hline. & . & . & . & $\cdot$ & . & . & . & . & . & . & + & + & . & . & . & . & . & . & . & . & . & + & + & + & + & + & 7 & 16,7 \\
\hline. & . & . & . & $\mathrm{r}$ & . & . & . & . & . & . & . & . & . & . & . & $\mathrm{r}$ & $\mathrm{r}$ & . & . & . & . & + & . & . & . & . & 6 & 14,3 \\
\hline+ & . & . & . & . & . & . & . & . & . & . & . & . & . & . & . & . & . & + & + & 1 & 1 & 2 & . & 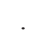 & . & . & 6 & 14,3 \\
\hline$\cdot$ & . & . & . & . & . & . & . & . & . & . & . & . & . & . & . & . & . & 1 & . & + & . & . & 1 & + & + & + & 6 & 14,3 \\
\hline$\cdot$ & . & . & . & . & . & . & . & . & . & . & . & . & . & $\mathrm{r}$ & + & + & . & $\mathrm{r}$ & $\mathrm{r}$ & . & . & . & . & 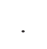 & . & . & $y$ & 11,9 \\
\hline$\cdot$ & + & . &. & $\cdot$ & . & . & . & . & . & . & . & . & . & . & . & . & . & 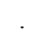 & . & . & . & . & 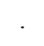 & $\mathrm{r}$ & + & $\mathrm{r}$ & 4 & 9,52 \\
\hline . & . & + & . & . & . & . & . & . & . & . & . & . & . & . & 1 & . & . & $\mathrm{r}$ & . & . & . & . & . &. & + & . & 4 & 9,52 \\
\hline . & . & . & . & . & . &. & . & . & . & . & + & . & . & . & . & . & . & + & . & . & . & + & . & . & . & + & 4 & 9,5 \\
\hline. & . & . & . & . & . & . & . & . & $\mathrm{r}$ & . & . & . & . & . & . & . & . & . &. & . & . & . & . & . & . & . & 3 & 7,1 \\
\hline$\cdot$ & . & . & . & . & . & . & . & . & . & . & . & . & . & . & . & + & + & . & . & . & . & . & . & . & . & . & 3 & 7,1 \\
\hline . & . & + & . & . & . & + & . & + & . & . & . & . & . & . & . & . & . & . & . & . & . & . & . & . & . & ${ }^{\circ}$ & 3 & 7,1 \\
\hline . & . & . & . & . & . & . & . & . & . & . & . & + & + & . & . & . & + & . & . & . & - & . & . & . & . & . & 3 & 7,1 \\
\hline$\cdot$ & . & . & + & . & . & . & . & + & . & . & . & . & . & . & . & . & . & . & . & . & . & . & . & . & . & $\cdot$ & & 4,7 \\
\hline$\cdot$ & . & . & . & . & . & . & . & . & . & + & + & . & . & . & . & . & . & . & . & . & . & . & . & . & . & . & 2 & 4,7 \\
\hline . & . & . & . & . & . & . & . & . & . & . & . & . & . & . & . & + & + & . & . & . & . & . & . & . & . & . & 2 & 4,76 \\
\hline$\cdot$ & . & . & . & . & . &. & . & . & . & . & . & . & . & . & . & . & . & . & . & . & . & + & . & . & $\cdot$ & 1 & 2 & $4, \pi$ \\
\hline . & . & . & . & . & . & . & . & . & . & . & . & . & . & . & . & . & . & . & . & . & . & . & + & + & . & . & 2 & 4,7 \\
\hline+ & . & $\cdot$ & $\cdot$ & $\cdot$ & . & $\cdot$ & $\cdot$ & $\cdot$ & . & + & 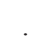 & + & $\mathrm{r}$ & 1 & $\cdot$ & $\mathrm{r}$ & . & + & + & $\cdot$ & + & . & + & $\cdot$ & $\cdot$ & 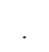 & 2 & 47,6 \\
\hline+ & . & . & . & + & . & + & + & . & + & + & . & . & $\mathrm{r}$ & + & + & + & $\mathrm{r}$ & . & + & + & 1 & . & + & 1 & + & $\cdot$ & 18 & 42,9 \\
\hline. & . & + & . & . & . & . & . & . & . & . & . & . & . & . & . & . & . & . & . & . & . & . & . & . & $\cdot$ & . & 1 & 23,8 \\
\hline. & . & . & . & . & . & . & 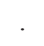 & . & . & . & . & . & . & . & . & . & . & . & . & + & . & . & 1 & + & 1 & + & 6 & 14,3 \\
\hline$\cdot$ & . & . & + & . & . & + & + & . & + & . & . & . & . & . & . & . & . & . & . & . & . & . & . & . & $\mathrm{r}$ & 1 & 6 & 14,3 \\
\hline 1 & . & . & . & . & . & . & . & . & . & . & . & . & + & . & . & . & . & 1 & . & . & . & . & . & . & . & . & 5 & 11,9 \\
\hline$\cdot$ & . & . & . & . & . & . & . & . & . & . & . & . & . & . & . & . & . & . & . & . & - & . & . & . & . & . & 5 & 11,9 \\
\hline+ & . & . & . & . & . & . & . & $\cdot$ & . & . & . & $\mathrm{r}$ & + & . & . & . & . & . & . & . & . & . & . & . & . & . & 5 & 11,9 \\
\hline$\cdot$ & . & . & . & . & . & . & . & . & . & . & . & . & . & . & . & . & . & + & + & . & . & . & + & . & + & . & 4 & 9,5 \\
\hline. & . & + & . & . & . & . & . & . & . & . & . & . & . & . & . & . & . & . & . & . & . & . & . & . & . & . & 3 & 7,1 \\
\hline. & . & . & . & . & + & . & . & . & . & . & . & . & . & . & . & . & . & $\mathrm{r}$ & . & . & . & . & . & . & . & . & 3 & 7,1 \\
\hline$\cdot$ & . & . & . & . & . & . & + & 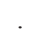 & . & . & . & . & + & $\mathrm{r}$ & . & . & . & . & . & . & . & . & . & . & . & . & 3 & 7,1 \\
\hline. & . & . & . & . & + & . & . & . & . & . & . & . & . & . & . & . & . & . & . & . & . & . & . & . & . & . & 2 & 4,76 \\
\hline. & . & . & . & + & + & . & . & . & . & . & . & . & . & . & . & . & . & . & . & . & . & . & . & . & . & . & 2 & 4,7 \\
\hline . & . & . & . & $\mathrm{r}$ & . & . & . & . & + & . & . & . & . & . & . & . & . & . & . & . & . & . & . & . & . & . & 2 & 4,7 \\
\hline . & . & . & . & . & . & . & . & . & . & . & . & . & . & . & & . & . & . & + & + & . & . & . & . & . & . & 2 & 4,76 \\
\hline 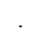 & . & . & . & . & . & . & . & . & . & . & . & . & . & . & . & . & . & . & . & + & . & . & $\mathrm{r}$ &. & . & . & 2 & 4,7 \\
\hline+ & 1 & 1 & $\cdot$ & $\cdot$ & . & + & . & ${ }^{\top}$ & ${ }^{\top}$ & . & 1 & . & 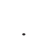 & . & $\cdot$ & ${ }^{\circ}$ & $\cdot$ & 1 & 1 & $T$ & + & + & + & + & $T^{\top}$ & $T^{\top}$ & 2 & 66,7 \\
\hline 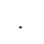 & . & . & + & + & + & + & . & . & + & + & 1 & 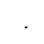 & . & . & $\cdot$ & ${ }^{\circ}$ & $\cdot$ & 1 & 1 & 2 & 3 & + & 1 & . & 2 & 2 & 18 & 42,9 \\
\hline+ & 1 & . & . & . & . & . & . & + & . & + & . & . & . & + & + & 1 & + & + & + & . & + & + & + & + & + & + & 16 & 38,1 \\
\hline . & + & + & + & . & . & . & + & + & . & + & $\mathrm{r}$ & . & . & . & . & . & . & . & . & + & + & + & + & + & + & + & 14 & 33,3 \\
\hline & + & + & & & . & . & 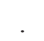 & 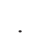 & 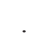 & 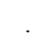 & + & 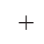 & & & + & & & 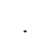 & 1 & + & & 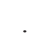 & + & & 2 & 1 & 13 & 1 \\
\hline
\end{tabular}




\begin{tabular}{|c|c|c|c|c|c|c|c|c|c|c|c|c|c|c|c|c|c|}
\hline & & Relevé number & 1 & 2 & 3 & 4 & 5 & 6 & $7 *$ & 8 & 9 & 10 & 11 & 12 & 13 & 14 & 15 \\
\hline$\overline{\mathrm{NP}}$ & S Medit.-Subatl. & Rosa arvensis & . & . & . & . & . & . & . & . & . & . & . & . & . & . & + \\
\hline G rhiz & Eurimedit. & Cephalanthera damasonium & . & . & . & . & . & . & . & . & . & . & . & . & . & . & . \\
\hline H caesp & Orof. SE Europ. & Luzula sylvatica subsp. sylvatica & . & . & . & . & . & . & . & . & . & . & . & . & . & . & . \\
\hline H rept & Europ.-Caucas. & Ajuga reptans & . & . & . & . & . & . & . & . & + & + & . & + & . & . & . \\
\hline H caesp & Paleotemp. & Brachypodium sylvaticum subsp. sylvaticum & . & . & . & . & . & . & . & . & . & . & . & . & . & . & . \\
\hline G rhiz & SE Europ. & Anemone apennina subsp. apennina & . & . & . & . & . & . & . & + & + & + & . & . & . & + & + \\
\hline H scap & Europ.-Caucas. & Hieracium racemosum & . & . & . & . & . & . & . & . & . & . & . & . & . & . & . \\
\hline H scap & Eurosib. & Hieracium murorum & . & . & . & . & . & . & . & . & . & . & + & . & . & . & . \\
\hline $\mathrm{H}$ ros & Eurimedit. & Potentilla micrantha & . & . & . & . & . & . & . & . & . & . & . & . & . & . & . \\
\hline $\mathrm{H}$ ros & Europ.-Caucas. & Primula vulgaris subsp. vulgaris & . & . & . & . & . & . & . & . & . & . & . & . & . & . & . \\
\hline H scap & Circumbor. & Geum urbanum & . & . & . & . & . & . & . & . & . & . & $\cdot$ & . & . & $\cdot$ & + \\
\hline H scap & Eurasiat. & Cruciata glabra subsp. glabra & . & . & . & . & . & . & . & . & . & . & . & . & . & . & . \\
\hline G bulb & S Europ. & Cyclamen hederifolium subsp. hederifolium & . & . & . & . & . & . & . & . & . & . & . & . & . & . & . \\
\hline \multirow[t]{2}{*}{ H scap } & Circumbor. & Solidago virgaurea subsp. virgaurea & . & . & . & . & . & + & . & . & . & . & $\cdot$ & . & . & . & . \\
\hline & & Accompanying taxa & & & & & & & & & & & & & & & \\
\hline T scap & Eurasiat. & Moehringia trinervia & . & . & . & . & . & . & . & . & . & . & . & . & . & . & . \\
\hline T scap & Paleotemp. & Silene conica & . & . & . & . & . & . & . & . & . & . & . & . & . & . & . \\
\hline H scap & Eurasiat. & Lamium maculatum & . & . & + & . & . & . & . & . & . & . & . & . & . & + & + \\
\hline H rept & Eurosib. & Fragaria vesca subsp. vesca & . & . & . & . & . & . & . & . & . & . & $\cdot$ & . & . & . & . \\
\hline G bulb & Eurasiat. & Ranunculus ficaria subsp. bulbifer & . & . & . & . & . & . & . & + & + & + & . & . & . & . & . \\
\hline H caesp & Endem. & Sesleria nitida (s.1.) & . & . & . & . & + & . & . & . & . & . & . & . & . & . & . \\
\hline H bienn & Eurasiat. & Myosotis nemorosa & . & . & . & . & . & . & . & . & . & . & + & . & + & + & . \\
\hline G bulb & Eurimedit. & Crocus vernus subsp. vernus & . & . & . & . & . & . & . & . & . & . & + & . & + & . & . \\
\hline H scap & Endem. & Campanula micrantha & . & . & . & . & . & . & . & . & . & . & . & . & . & + & . \\
\hline H scap & C Europ. & Senecio ovatus subsp. alpestris & . & . & . & . & . & . & . & . & . & . & . & . & . & . & . \\
\hline G rhiz & Eurosib. & Aegopodium podagraria & . & . & . & . & . & . & . & . & . & . & . & . & . & . & . \\
\hline & & Sporadic taxa & 0 & 0 & 0 & 1 & 0 & 2 & 1 & 0 & 0 & 0 & 1 & 0 & 3 & 1 & 0 \\
\hline
\end{tabular}

Table 9 (Tabela 9): Cardamino kitaibelii-Fagetum sylvaticae Ubaldi, Zanotti, Puppi, Speranza et Corbetta ex Ubaldi 1993 anemonetosum nemorosae subass. nova Festuca heterophylla variant.

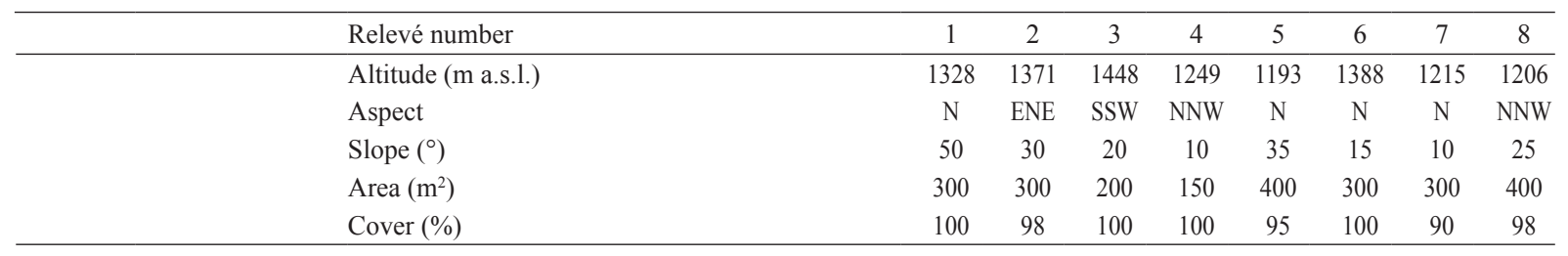

\begin{tabular}{|c|c|c|}
\hline G rhiz & Circumbor. & $\begin{array}{l}\text { Characteristic species of the ass } \\
\text { Hepatica nobilis }\end{array}$ \\
\hline G rhiz & Circumbor. & Anemone nemorosa \\
\hline H caesp & Orof. SE Europ. & Luzula sylvatica subsp. sylvatica \\
\hline G rhiz & SE Europ. & Cardamine enneaphyllos* \\
\hline P scap & SE Europ. & Acer opalus subsp. obtusatum \\
\hline G rhiz & Circumbor. & Adoxa moschatellina* \\
\hline H scap & Eurasiat. & Epilobium montanum $*$ \\
\hline G rhiz & Paleotemp. & Epipactis leptochila \\
\hline G rhiz & Eurasiat. & Lathyrus vernus subsp. vernus* \\
\hline G rhiz & Eurasiat. & Polystichum aculeatum* \\
\hline G rhiz & Orof. SE Europ. & Cardamine kitaibelii* \\
\hline
\end{tabular}




\begin{tabular}{|c|c|c|c|c|c|c|c|c|c|c|c|c|c|c|c|c|c|c|c|c|c|c|c|c|c|c|c|c|}
\hline 16 & 17 & 18 & 19 & 20 & 21 & 22 & 23 & 24 & 25 & 26 & 27 & 28 & 29 & 30 & 31 & 32 & 33 & 34 & 35 & 36 & 37 & 38 & 39 & 40 & 41 & 42 & Pres & Freq \\
\hline . & . & . & . & . & . & . & . & . & . & + & + & + & + & + & + & + & + & $\mathrm{r}$ & $r$ & . & . & 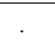 & + & . & + & . & 13 & 31 \\
\hline+ & . & + & + & + & + & . & + & + & $r$ & + & $\mathrm{r}$ & . & . & . &. & . & . & . & . & . & . & $\mathrm{r}$ & + & . & $\mathrm{r}$ & . & 13 & 31 \\
\hline . & . & . & + & . & . & . & + & + & . & + & + & . & + & $\mathrm{r}$ & . & $\mathrm{r}$ & . & + & + & . & . & . & + & . & $\mathrm{r}$ & . & 12 & 28,6 \\
\hline . & . & . & . & . & . & . & + & . & . & . & . & + & + & + & . & + & + & . & . & . & . & . & . & . & . & . & 9 & 21,4 \\
\hline . & + & . & . & . & . & + & + & + & + & + & . & . & . & . &. & . & . & . & . & . & . & . & . & + & . & + & 8 & 19 \\
\hline . & . & + & . & . & . & . & . & . & . & . & . & . & . & . & . & . & . & . & . & . & . & . & . & . & . & . & 6 & 14,3 \\
\hline . & . & . & . & . & . & . & . &. & + & . & . & $\mathrm{r}$ & + & + &. & . & 1 & . & . & . & . & . & . & . &. & . & 5 & 11,9 \\
\hline . & . & . & . & . & . & . & . &. & . & . & + & . & . & . &. & . & . & . & . & . & . & . & $\mathrm{r}$ & . & $\mathrm{r}$ & . & 4 & 9,52 \\
\hline . & . & . & . & . & . & . & . & . & . & . & . & $\mathrm{r}$ & $\mathrm{r}$ & . &. & + & $\mathrm{r}$ & . & . & . & . & . & . & . & . & . & 4 & 9,52 \\
\hline . & . & . & . & . & . &. & . & . & . & . & . & . & . & . & . & . & . & + & + & + & . & . & . & . & $\mathrm{r}$ & . & 4 & 9,52 \\
\hline . & . & . & . & . & . &. & . & . & . & . & . & . & . & . &. & . & . & . & . & + & . & . & $\mathrm{r}$ & . & . & . & 3 & 7,14 \\
\hline . & . & . & . & . & . & . & . & . & . & . & . & . & + & $\mathrm{r}$ &. & . & . & $\mathrm{r}$ & . & . & . & . & . & . & . & . & 3 & 7,14 \\
\hline . & $\cdot$ & $\cdot$ & $\cdot$ & . & . & $\cdot$ & $\cdot$ & . & $\cdot$ & . & . & . & . & . & . & . & . & . & . & . & . & . & + & + & + & . & 3 & 7,14 \\
\hline . & . & . & . & . & . &. & . & . & 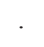 & . & . & . & . & . & . & . & 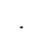 & . & . & . & . & . & $\mathrm{r}$ & . & . & . & 2 & 4,76 \\
\hline . & + & . & . & + & . & + & + & . & + & . & . & $\mathrm{r}$ & + & $\mathrm{r}$ & . & . & . & . & . & . & . & . & . & . &. & . & 8 & 19 \\
\hline . & . & . & . & . & . & . & . & . & . & $\mathrm{r}$ & + & . & . & + & $\mathrm{r}$ & . & . & . & . & . & . & . & $\mathrm{r}$ & + & $\mathrm{r}$ & $\mathrm{r}$ & 8 & 19 \\
\hline . & . & + & . & . & . & . & . & . & . & . & . & . & . & . & . & + & + & . & . & . & . & . & . & . & . & . & 6 & 14,3 \\
\hline . & . & . & . & . & . & . & . & . & . & . & . & . & . & $\mathrm{r}$ & + & . & . & + & . & + & . & . & $\mathrm{r}$ & . & . & . & 5 & 11,9 \\
\hline . & . & + & . & . & . & . & . & . & 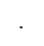 & . & . & . & 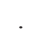 & . & . & . & 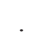 & . & . & . & . & . & . & . & . & . & 4 & 9,52 \\
\hline+ & . & . & . & . & . & . & + & . & . & . & . & . & . & . & . & . & . & . & . & . & . & . & . & . & . & . & 3 & 7,14 \\
\hline . & . & . & . & . & . &. & . & . & 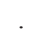 & . & . & 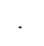 & 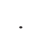 & . & 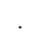 & . & 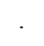 & . & . & . & . & . & . & . &. & . & 3 & 7,14 \\
\hline . & . & . & . & . & . & . & . & . & . & . & . & . & . & . &. & . & . & . & . & . & . & . & . & . & $r$ & . & 3 & 7,14 \\
\hline+ & . & . & . & . & . & . & . & . & + & . & . & 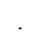 & 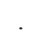 & . & . & . & . & . & . & . & . & . & . & . & . & . & 3 & 7,14 \\
\hline 1 & . & . & . & . & . & . & 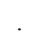 & . & 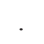 & . & . & . & . & . & . & . & . & . & . & + & + & . & 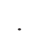 & . & . & . & 3 & 7,14 \\
\hline. & . & . & . & . & . & . & . & . & . & . & $\cdot$ & . & . & . & . & + & $\cdot$ & 2 & $\cdot$ & . & . & + & . & . & . & . & 3 & 7,14 \\
\hline
\end{tabular}

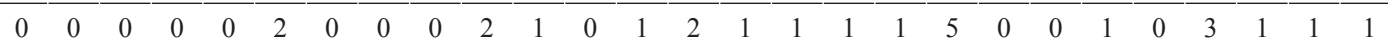

\begin{tabular}{|c|c|c|c|c|c|c|c|c|c|c|c|c|c|c|c|c|c|c|}
\hline 9 & 10 & 11 & 12 & 13 & 14 & 15 & $16^{*}$ & 17 & 18 & 19 & 20 & 21 & 22 & 23 & 24 & 25 & & \\
\hline 1265 & 1390 & 1342 & 1353 & 1050 & 1193 & 1200 & 1345 & 1360 & 1345 & 1440 & 1240 & 1280 & 1350 & 1320 & 1400 & 1450 & & \\
\hline $\mathrm{NE}$ & ENE & WNW & WSW & W & $\mathrm{N}$ & NNE & WNW & WNW & $\mathrm{N}$ & WNW & $\mathrm{N}$ & $\mathrm{N}$ & E & $\mathrm{N}$ & ESE & WNW & $\mathrm{P}$ & $\mathrm{F}$ \\
\hline 31 & 35 & 45 & 33 & $35 / 40$ & 20 & 40 & 30 & 30 & 45 & 35 & 50 & 25 & 60 & 55 & 30 & 15 & $\mathrm{r}$ & r \\
\hline 150 & 150 & 400 & 400 & 400 & 300 & 400 & 400 & 200 & 350 & 200 & 200 & 400 & 400 & 400 & 300 & 350 & $\mathrm{~s}$ & $q$ \\
\hline 100 & 100 & 93 & 95 & 100 & 100 & 98 & 100 & 100 & 100 & 95 & 95 & 100 & 100 & 100 & 100 & 100 & & \\
\hline
\end{tabular}

of the subassociation anemonetosum nemorosae

\begin{tabular}{|c|c|c|c|c|c|c|c|c|c|c|c|c|c|c|c|c|c|}
\hline+ & . & . & . & + & 1 & + & 1 & + & 1 & + & 1 & + & + & $\mathrm{r}$ & + & + & 20 \\
\hline . & + & 1 & + & + & + & + & + & + & . & + & + & + & + & + & . & + & 20 \\
\hline+ & . & . & . & 1 & + & + & + & . & . & + & . & + & + & $\mathrm{r}$ & . & + & 16 \\
\hline+ & . & + & . & + & . & + & . & + & + & . & + & 1 & . & + & . & + & 15 \\
\hline . & 1 & + & + & . & 1 & . & + & 1 & + & . & 1 & . & . & . & . & . & 12 \\
\hline+ & + & . & . & + & + & . & + & . & + & + & . & . & . & . & + & + & 12 \\
\hline+ & + & . & + & . & . & + & . & + & . & . & . & . & + & . & $\mathrm{r}$ & . & 10 \\
\hline+ & + & . & + & . & . & + & + & . & . & . & + & $\mathrm{r}$ & $\mathrm{r}$ & $\mathrm{r}$ & $\mathrm{r}$ & . & 10 \\
\hline . & . & + & + & . & . & . & . & . & . & . & . & $\mathrm{r}$ & + & + & + & + & 9 \\
\hline . & $\cdot$ & + & . & + & . & + & . & . & . & + & . & + & . & + & . & . & 8 \\
\hline . & + & . & . & . & + & . & . & . & . & . & . & . & . & + & . & . & 6 \\
\hline
\end{tabular}




\begin{tabular}{|c|c|c|}
\hline & & Species of the Festuca heterophylla varian \\
\hline H caesp & Europ.-Caucas. & Festuca heterophylla \\
\hline \multirow[t]{2}{*}{ H scap } & Eurosib. & Hieracium murorum \\
\hline & & Character species of the alliance Aremon \\
\hline G rhiz & Europ.-Caucas. & Anemone ranunculoides* \\
\hline H scap & Orof. S Europ. & Saxifraga rotundifolia subsp. rotundifolia* \\
\hline H scap & NE Medit. mont. & Adenostyles glabra subsp. glabra \\
\hline G rhiz & N Medit. mont. & Geranium nodosum \\
\hline G bulb & Paleotemp. & Dactylorhiza maculata subsp. fuchsii \\
\hline H scap & Europ.-Caucas. & Ranunculus lanuginosus* \\
\hline
\end{tabular}

\begin{tabular}{|llllll|}
\hline+ & + & 1 & + & + & + \\
& 1 & 1 &. & 1 & + \\
\hline
\end{tabular}

\section{Charact. species of the order Fagetalia sylvaticae}

$\begin{array}{lll}\text { P scap } & \text { C Europ. } & \text { Fagus sylvatica } \text { subsp. sylvatica } \\ \text { H scap } & \text { Europ.-Caucas. } & \text { Lactuca muralis } \\ \text { G rhiz } & \text { Eurasiat. } & \text { Neottia nidus-avis } \\ \text { H scap } & \text { Europ.-Caucas. } & \text { Prenanthes purpurea } \\ \text { G bulb } & \text { Europ.-Caucas. } & \text { Scilla bifolia } \\ \text { G rhiz } & \text { Europ.-Caucas. } & \text { Galium odoratum } \\ \text { H scap } & \text { Eurosib. } & \text { Viola reichenbachiana } \\ \text { H caesp } & \text { Circumbor. } & \text { Poa nemoralis subsp. } \text { nemoralis } \\ \text { H scap } & \text { Paleotemp. } & \text { Campanula trachelium subsp. trachelium } \\ \text { H ros } & \text { Paleotrop. } & \text { Polypodium interjectum } \\ \text { H caesp } & \text { Eurimedit. } & \text { Luzula forsteri } \\ \text { G bulb } & \text { Eurosib. } & \text { Gagea lutea } \\ \text { Ch rept } & \text { Circumbor. } & \text { Orthilia secunda } \\ \text { H scap } & \text { Paleotemp. } & \text { Heracleum sphondylium subsp. ternatum } \\ \text { G rhiz } & \text { Europ.-Caucas. } & \text { Epipactis microphylla } \\ \text { G rhiz } & \text { Eurasiat. } & \text { Cephalanthera rubra } \\ \text { G rhiz } & \text { C Europ. } & \text { Euphorbia dulcis } \\ \text { G rhiz } & \text { C Europ. } & \text { Cardamine bulbifera } \\ \text { G bulb } & \text { Eurasiat. } & \text { Lilium martagon } \\ \text { H scap } & & \text { Stellaria nemorum (s.l.) } \\ \text { P scap } & \text { Europ.-Caucas. } & \text { Acer platanoides } \\ \text { T scap } & \text { Subcosmop. } & \text { Geranium robertianum } \\ \text { H caesp } & \text { Subatl. } & \text { Festuca altissima } \\ \text { P caesp } & \text { Europ. } & \text { Sorbus aucuparia } \text { subsp. aucuparia } \\ \text { P scap } & \text { Europ.-Caucas. } & \text { Acer pseudoplatanus } \\ \text { NP } & \text { Eurosib. } & \text { Daphne mezereum } \\ \text { P caesp } & \text { Orof. S Europ. } & \text { Laburnum alpinum } \\ \text { Ch suffr } & \text { Europ.-Caucas. } & \text { Euphorbia amygdaloides subsp. amygdaloides } \\ \text { H scap } & \text { Europ.-Caucas. } & \text { Lamium galeobdolon subsp. montanum } \\ \text { P scap } & \text { Pont. } & \text { Prunus avium subsp. avium } \\ \text { NP } & \text { Eurosib. } & \text { Ribes alpinum } \\ \text { G rhiz } & \text { Eurasiat. } & \text { Actaea spicata } \\ \text { H scap } & \text { Orof. S Europ. } & \text { Valeriana tripteris subsp. tripteris } \\ \text { G rhiz } & \text { Europ.-Caucas. } & \text { Mercurialis perennis } \\ \text { G rhiz } & \text { Circumbor. } & \text { Corallorhiza trifida } \\ \text { P scap } & \text { Eurasiat. } & \text { Polygonatum verticillatum } \\ & \text { Europ.-Caucas. } & \text { Stellaria holostea } \text { subsp. holostea } \\ \text { Paxus baccata }\end{array}$

$\begin{array}{llllllll}5 & 5 & 5 & 5 & 5 & 5 & 5 & 5 \\ + & & + & + & + & + & + & +\end{array}$

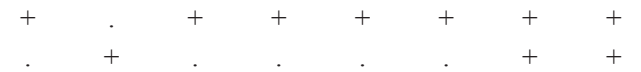

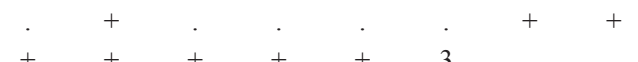

$\begin{array}{llll}+ & + & + & +\end{array}$

$\begin{array}{llll}+ & + & + & + \\ 1 & + & +\end{array}$ 


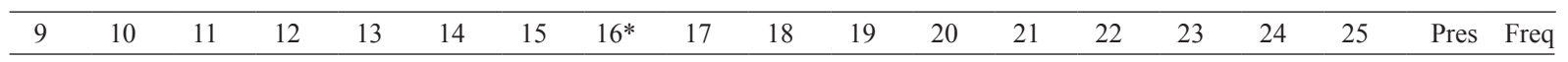

\section{belii-Fagenion sylvaticae}

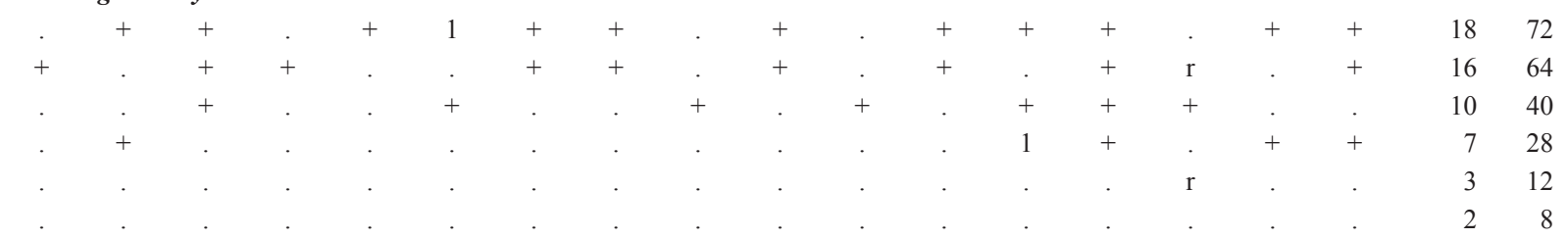

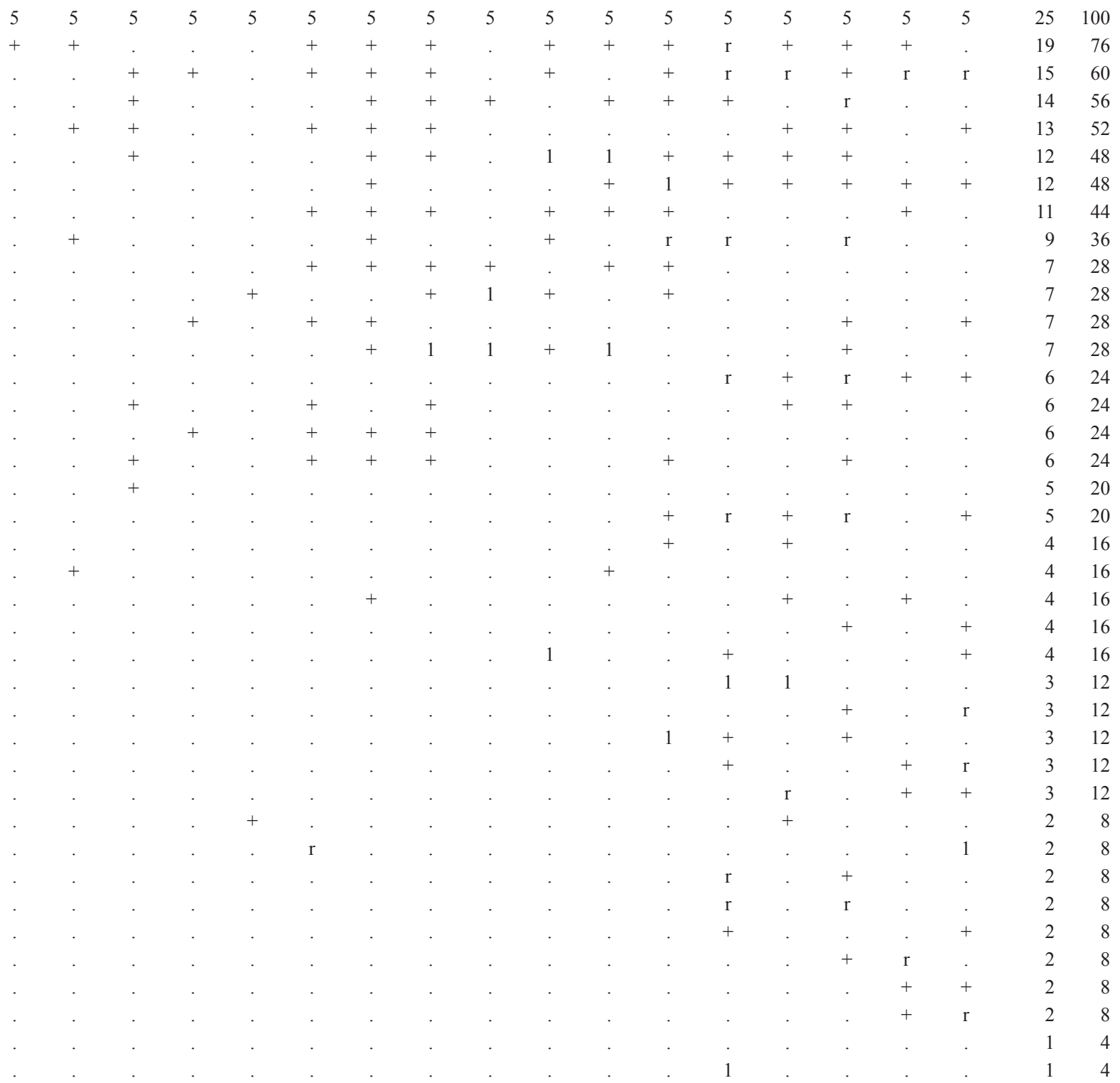




\begin{tabular}{|c|c|c|c|c|c|c|c|c|c|c|}
\hline & & Relevé number & 1 & 2 & 3 & 4 & 5 & 6 & 7 & 8 \\
\hline P caesp & S Europ.-Sudsib. & Laburnum anagyroides subsp. anagyroides & 2 & + & . & . & . & . & 1 & . \\
\hline H scap & Endem. & Digitalis lutea subsp. australis & . & . & + & . & . & . & $\mathrm{r}$ & . \\
\hline P scap & S Europ.-Sudsib. & Fraxinus ornus subsp. ornus & . & . & . & . & + & . & + & . \\
\hline H caesp & Eurasiat. & Carex digitata & . & . & . & . & . & . & . & . \\
\hline G rhiz & Endem. & Helleborus bocconei subsp. bocconei & + & . & + & . & . & . & + & + \\
\hline G rhiz & Eurasiat. & Cephalanthera longifolia & . & + & + & . & . & . & . & . \\
\hline$P$ caesp & Europ.-Caucas. & Lonicera xylosteum & . & . & . & + & . & . & + & . \\
\hline $\mathrm{H}$ caesp & Subatl. & Brachypodium rupestre & . & + & . & . & . & . & . & . \\
\hline H caesp & Euromedit. & Poa sylvicola & . & . & . & . & . & + & . & . \\
\hline G rhiz & Paleotemp. & Epipactis helleborine (s.1.) & . & . & . & . & . & . & . & . \\
\hline H scap & C Europ. & Melittis melissophyllum subsp. melissophyllum & . & . & . & . & . & . & . & . \\
\hline P caesp & C Europ. & Viburnum lantana & . & . & . & . & . & . & . & . \\
\hline NP & & Emerus majus (s.1.) & . & . & . & + & . & . & . & . \\
\hline G bulb & Orof. C Europ. & Lilium bulbiferum subsp. croceum & . & . & . & . & . & . & . & . \\
\hline$P$ caesp & Medit. mont. & Euonymus latifolius & . & . & . & . & . & . & . & . \\
\hline \multirow[t]{2}{*}{ P scap } & SE Europ. & Ostrya carpinifolia & . & . & . & . & . & . & . & . \\
\hline & & Charact. species of the class Querco-Fagetea & & & & & & & & \\
\hline G bulb & Europ.-Caucas. & Galanthus nivalis subsp. nivalis & . & . & + & . & 1 & 1 & + & . \\
\hline G rhiz & Eurimedit. & Cephalanthera damasonium & . & . & . & . & . & . & + & . \\
\hline H scap & Europ.-Caucas. & Hieracium racemosum & + & . & . & . & . & 1 & + & . \\
\hline$P$ caesp & Subatlant. & Daphne laureola & + & + & + & 1 & + & . & . & . \\
\hline NP & N Eurimedit. & Rubus hirtus & + & + & . & . & . & . & . & . \\
\hline H caesp & Paleotemp. & Melica uniflora & . & . & . & . & + & + & . & . \\
\hline H scap & Paleotemp. & Sanicula europaea & + & . & 2 & . & . & . & . & . \\
\hline NP & S Medit.-Subatl. & Rosa arvensis & . & . & + & + & . & . & . & . \\
\hline $\mathrm{H}$ ros & Europ.-Caucas. & Primula vulgaris subsp. vulgaris & . & . & . & . & . & . & . & . \\
\hline H scap & Circumbor. & Solidago virgaurea subsp. virgaurea & . & . & . & . & . & . & . & . \\
\hline G bulb & S Europ. & Cyclamen hederifolium subsp. hederifolium & + & + & . & . & . & . & . & . \\
\hline H rept & Europ.-Caucas. & Ajuga reptans & . & . & . & . & . & . & . & . \\
\hline $\mathrm{H}$ ros & NE Stenomedit. & Aremonia agrimonoides subsp. agrimonoides & . & . & . & . & . & . & . & . \\
\hline G rhiz & S Europ.-Sudsib. & Lathyrus venetus & . & . & . & + & + & . & . & . \\
\hline G rhiz & SE Europ. & Anemone apennina subsp. apennina & . & . & . & . & . & . & . & . \\
\hline G bulb & Paleotemp. & Platanthera bifolia & . & . & + & . & . & . & . & . \\
\hline H scap & Eurasiat. & Cruciata glabra subsp. glabra & . & . & . & . & . & . & . & . \\
\hline \multirow[t]{2}{*}{ P lian } & Eurimedit. & Hedera helix subsp. helix & . & . & . & . & . & . & . & . \\
\hline & & Accompanying taxa & & & & & & & & \\
\hline T scap & Eurasiat. & Moehringia trinervia & + & + & + & . & . & + & . & . \\
\hline H scap & Endem. & Campanula micrantha & . & . & + & . & + & + & . & . \\
\hline H caesp & Endem. & Sesleria nitida (s.1.) & . & . & . & . & . & . & . & . \\
\hline H scap & Sudsib. & Veronica chamaedrys subsp. chamaedrys & . & + & + & . & . & + & . & . \\
\hline$P$ caesp & Circumbor. & Juniperus communis subsp. communis & . & . & + & . & . & . & . & . \\
\hline H rept & Eurosib. & Fragaria vesca subsp. vesca & . & . & + & . & . & . & . & . \\
\hline \multirow[t]{2}{*}{ P caesp } & Medit. mont. & Amelanchier ovalis subsp. ovalis & . & . & . & . & . & . & . & . \\
\hline & & Sporadic taxa & 6 & 0 & 5 & 2 & 2 & 0 & 2 & 1 \\
\hline
\end{tabular}




\begin{tabular}{|c|c|c|c|c|c|c|c|c|c|c|c|c|c|c|c|c|c|c|}
\hline 9 & 10 & 11 & 12 & 13 & 14 & 15 & $16^{*}$ & 17 & 18 & 19 & 20 & 21 & 22 & 23 & 24 & 25 & Pres & $\overline{\text { Freq }}$ \\
\hline . & . & . & . & . & . & + & . & 1 & + & . & . & . & . & . & . & . & 6 & $\overline{24}$ \\
\hline . & . & . & . & . & + & + & + & . & . & . & $\mathrm{r}$ & . & . & . & . & . & 6 & 24 \\
\hline . & . & . & . & . & + & . & + & + & . & . & $\mathrm{r}$ & . & . & . & . & . & 6 & 24 \\
\hline . & . & . & . & . & + & . & . & + & . & . & . & + & + & + & . & + & 6 & 24 \\
\hline . & . &. & . & . & . & . & . & . & . & . & + & . & . & . & . & . & 5 & 20 \\
\hline . & . & . & . & . & . & . & . & . & + & + & + & . & . & . & . & . & 5 & 20 \\
\hline . & . & . & . & . & + & . & . & . & . & . & . & . & . & . & . & + & 4 & 16 \\
\hline . & . & + & . & . & . & 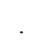 & . & . & . & + & . & . & . & . & . & . & 3 & 12 \\
\hline . & . & . & . & . & . & . & + & . & . & . & + & . & . & . & . & . & 3 & 12 \\
\hline . & . & + & + & . & . & . & . & . & . & . & . & . & . & . & . & $\mathrm{r}$ & 3 & 12 \\
\hline . & . & . & . & . & . & . & . & . & . & . & . & + & . & $\mathrm{r}$ & . & + & 3 & 12 \\
\hline . & . & . & . & . & . & . & . & . & . & . & . & . & + & . & + & + & 3 & 12 \\
\hline . & . & . & . & . & . & . & . & + & . & . & . & . & . & . & . & . & 2 & 8 \\
\hline . & + & . & . & . & . & . & . & . & . & . & . & . & $\mathrm{r}$ & . & . & . & 2 & 8 \\
\hline . & . & . & . & . & . & . & . & . & . & . & . & + & . & . & . & $r$ & 2 & 8 \\
\hline . & . & . & . & . & . & . & 1 & . & . & . & . & . & . & . & . & . & 1 & 4 \\
\hline . & 1 & + & + & 1 & + & + & + & . & . & . & 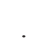 & + & + & 1 & . & + & 15 & 60 \\
\hline+ & 1 & . & . & . & . & + & + & + & + & + & + & $\mathrm{r}$ & $\mathrm{r}$ & . & + & $\mathrm{r}$ & 13 & 52 \\
\hline . & . & . & . & . & + & + & . & + & + & + & + & + & . & $\mathrm{r}$ & $\mathrm{r}$ & . & 12 & 48 \\
\hline . & . & . & + & . & . & + & . & 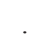 & . & + & + & + & . & + & . & . & 11 & 44 \\
\hline . & . & + & + & + & + & + & . & . & + & + & 1 & . & + & . & . & . & 11 & 44 \\
\hline . & . & . & . & . & + & . & . & . & . & . & . & $\mathrm{r}$ & + & + & + & + & 8 & 32 \\
\hline . & . & . & . & + & . & 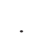 & . & 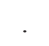 & . & 1 & + & 1 & + & . & . & . & 7 & 28 \\
\hline . & . & . & . & . & + & . & . & + & . & + & + & . & 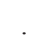 & . & + & . & 7 & 28 \\
\hline . & . & + & . & + & . & . & . & . & . & . & + & + & + & . & + & + & 7 & 28 \\
\hline . & . & . & . & . & . & + & + & + & . & . & . & . & $\mathrm{r}$ & $\mathrm{r}$ & 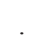 & . & 5 & 20 \\
\hline . & . & . & . & . & . & . & . & 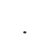 & 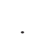 & . & . & $\mathrm{r}$ & + & . & . & . & 4 & 16 \\
\hline . & . & . & 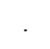 & . & . & . & + & . & + & . & + & . & . & . & . & + & 4 & 16 \\
\hline . & . & . & . & . & . & 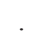 & . & . & . & . & . & + & + & . & + & + & 4 & 16 \\
\hline . & . & . & . & . & . & . & . & . & . & + & . & . & . & . & . & . & 3 & 12 \\
\hline . & . & . & . & + & + & 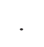 & . & . & . & 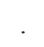 & . & . & . & . & 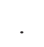 & + & 3 & 12 \\
\hline . & . & . & . & . & . & . & . & . & . & + & . & . & . & . & . & . & 2 & 8 \\
\hline . & . & . & . & . & . & 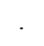 & $\mathrm{r}$ & . & . & + & . & . & . & . & 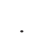 & . & 2 & 8 \\
\hline . & . & . & . & . & . & . & . & . & . & . & . & + & . & $\mathrm{r}$ & 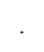 & . & 2 & 8 \\
\hline . & . & + & . & . & + & . & . & . & . & . & . & . & . & . & . & . & 6 & 24 \\
\hline . & . & . & . & . & . & 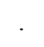 & . & . & . & + & . & . & . & . & 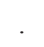 & . & 4 & 16 \\
\hline . & . & . & . & . & + & 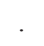 & + & + & . & 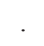 & + & . & . & . & . & . & 4 & 16 \\
\hline . & . & . & . & . & . & 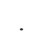 & . & . & . & 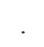 & . & . & . & . & 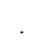 & . & 3 & 12 \\
\hline . & . & . & . & . & $\mathrm{r}$ & . & . & + & . & . & . & . & . & . & . & . & 3 & 12 \\
\hline . & . & . & . & . & . & 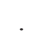 & . & + & . & . & + & . & . & . & 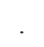 & . & 3 & 12 \\
\hline . & . & . & . & . & + & . & + & + & . & . & . & . & . & . & . & . & 3 & 12 \\
\hline 1 & 1 & 0 & 0 & 1 & 2 & 2 & 0 & 2 & 0 & 1 & 1 & 2 & 3 & 3 & 0 & 2 & & \\
\hline
\end{tabular}


Table 10 (Tabela 10): Cardamino kitaibelii-Fagetum sylvaticae Ubaldi, Zanotti, Puppi, Speranza et Corbetta ex Ubaldi 1993 corallorhizetosum trifidae subass. nova Moehringia trinervia variant.

\begin{tabular}{llccccccccccc}
\hline & Relevé number & 1 & 2 & 3 & 4 & 5 & 6 & 7 & $8^{*}$ & 9 & 10 & 11 \\
\hline Altitude (m a.s.1.) & 1650 & 1620 & 1630 & 1650 & 1350 & 1670 & 1640 & 1680 & 1600 & 1620 & 1660 \\
Aspect & $\mathrm{W}$ & $\mathrm{N}$ & $\mathrm{E}$ & $\mathrm{E}$ & $\mathrm{ENE}$ & $\mathrm{N}$ & $\mathrm{N}$ & $\mathrm{N}$ & $\mathrm{N}$ & $\mathrm{NW}$ & $\mathrm{N}$ \\
Slope $\left({ }^{\circ}\right)$ & 30 & 25 & 25 & 50 & 30 & 45 & 45 & 50 & 30 & 50 & 40 \\
Area $\left(\mathrm{m}^{2}\right)$ & 400 & 400 & 300 & 100 & 400 & 200 & 400 & 400 & 400 & 400 & 400 \\
& Cover $(\%)$ & 100 & 100 & 100 & 100 & 100 & 100 & 100 & 100 & 100 & 100 & 100 \\
\hline
\end{tabular}

\begin{tabular}{|c|c|c|}
\hline & & haracteristic species of the \\
\hline G rhiz & SE Europ. & Cardamine enneaphyllos* \\
\hline G rhiz & Circumbor. & Adoxa moschatellina* \\
\hline G rhiz & Eurasiat. & Lathyrus vernus subsp. vernus* \\
\hline G rhiz & Eurasiat. & Polystichum aculeatum* \\
\hline H scap & Eurasiat. & Epilobium montanum* \\
\hline G rhiz & Orof. SE Europ. & Cardamine kitaibelii* \\
\hline H scap & Paleotemp. & Silene dioica \\
\hline G rhiz & Circumbor. & Corallorhiza trifida \\
\hline G rhiz & Eurasiat. & Actaea spicata \\
\hline G rhiz & Circumbor. & Polystichum lonchitis \\
\hline
\end{tabular}

$\begin{array}{lll} & & \text { Species of the Moehringia trinervia var } \\ \text { H scap } & \text { Eurasiat. } & \text { Lamium maculatum } \\ \text { H bienn } & \text { Eurasiat. } & \text { Myosotis nemorosa } \\ \text { T scap } & \text { Eurasiat. } & \text { Moehringia trinervia } \\ \text { H scap } & \text { E Medit. mont. } & \text { Lamium garganicum subsp. laevigatum } \\ \text { G rhiz } & \text { Eurosib. } & \text { Aegopodium podagraria } \\ \text { H scap } & \text { Endem. } & \text { Campanula micrantha }\end{array}$

Charact. species of the alliance Aremonio-Fagion sylvaticae* and diff. of the suball. Cardamino kitaibelii-Fage-

$\begin{array}{lll}\text { H ros } & \text { NE Stenomedit. } & \text { Aremonia agrimonoides subsp. agrimonoides } \\ \text { H scap } & \text { NE Medit. mont. } & \text { Adenostyles glabra subsp. glabra } \\ \text { H scap } & \text { Endem. } & \text { Pulmonaria apennina } \\ \text { G rhiz } & \text { Europ.-Caucas. } & \text { Anemone ranunculoides* } \\ \text { H scap } & \text { Europ.-Caucas. } & \text { Ranunculus lanuginosus* } \\ \text { H scap } & \text { Orof. S Europ. } & \text { Saxifraga rotundifolia subsp. rotundifolia* }\end{array}$

\begin{tabular}{|c|c|c|c|c|c|c|c|c|c|c|}
\hline+ & + & + & 1 & 1 & 1 & 1 & + & + & 1 & 2 \\
\hline . & + & + & . & . & + & + & . & + & + & + \\
\hline+ & . & . & + & 1 & + & + & + & . & $\mathrm{r}$ & . \\
\hline . & . & . & . & + & 1 & + & + & + & + & + \\
\hline+ & . & + & . & . & + & + & . & + & . & + \\
\hline . & . & . & $\cdot$ & . & . & . & . & $\cdot$ & . & + \\
\hline+ & . & + & + & . & + & . & + & . & + & . \\
\hline . & + & . & + & + & . & + & + & + & . & $\cdot$ \\
\hline . & . & . & . & . & + & . & . & $\cdot$ & + & + \\
\hline+ & . & + & . & . & + & . & $\mathrm{r}$ & + & $r$ & \\
\hline
\end{tabular}

$\begin{array}{lll} & & \text { Charact. species of the order Fagetalia sylvaticae } \\ \text { P scap } & \text { C Europ. } & \text { Fagus sylvatica subsp. sylvatica } \\ \text { G rhiz } & \text { Europ.-Caucas. } & \text { Galium odoratum } \\ \text { G rhiz } & \text { C Europ. } & \text { Cardamine bulbifera } \\ \text { G bulb } & \text { Europ.-Caucas. } & \text { Corydalis cava subsp. cava } \\ \text { H scap } & \text { Europ.-Caucas. } & \text { Lactuca muralis } \\ \text { H caesp } & \text { Circumbor. } & \text { Poa nemoralis subsp. nemoralis } \\ \text { G bulb } & \text { Europ.-Caucas. } & \text { Scilla bifolia } \\ \text { H scap } & \text { Eurosib. } & \text { Viola reichenbachiana } \\ \text { H scap } & & \text { Stellaria nemorum } \text { (s.1.) } \\ \text { G rhiz } & \text { Eurasiat. } & \text { Neottia nidus-avis } \\ \text { H scap } & \text { Paleotemp. } & \text { Campanula trachelium subsp. trachelium } \\ \text { T scap } & \text { Subcosmop. } & \text { Geranium robertianum } \\ \text { G rhiz } & \text { Europ.-Caucas. } & \text { Epipactis microphylla } \\ \text { G rhiz } & \text { Subcosmop. } & \text { Dryopteris filix-mas } \\ \text { G bulb } & \text { Eurasiat. } & \text { Lilium martagon } \\ \text { Ch suffr } & \text { Europ.-Caucas. } & \text { Euphorbia amygdaloides subsp. amygdaloides } \\ \text { G rhiz } & \text { Eurasiat. } & \text { Cephalanthera rubra } \\ \text { H scap } & \text { Europ.-Caucas. } & \text { Prenanthes purpurea }\end{array}$




\begin{tabular}{cccccccccccccccccccccc}
\hline 12 & 13 & 14 & 15 & 16 & 17 & 18 & 19 & 20 & 21 & 22 & 23 & 24 & 25 & 26 & 27 & 28 & 29 & 30 & 31 & \\
\hline 1680 & 1600 & 1620 & 1630 & 1650 & 1650 & 1620 & 1700 & 1720 & 1710 & 1740 & 1740 & 1730 & 1710 & 1680 & 1680 & 1720 & 1710 & 1700 & 1650 & \\
$\mathrm{~N}$ & $\mathrm{~N}$ & $\mathrm{NNE}$ & $\mathrm{ENE}$ & $\mathrm{N}$ & $\mathrm{N}$ & $\mathrm{N}$ & $\mathrm{W}$ & $\mathrm{N}$ & $\mathrm{WSW}$ & $\mathrm{N}$ & $\mathrm{N}$ & $\mathrm{N}$ & $\mathrm{W}$ & $\mathrm{SW}$ & $\mathrm{S}$ & $\mathrm{SW}$ & $\mathrm{S}$ & $\mathrm{S}$ & $\mathrm{S}$ & $\mathrm{P}$ & $\mathrm{F}$ \\
20 & 60 & 30 & 30 & 45 & 45 & 25 & 40 & 40 & 45 & 40 & 20 & 30 & 35 & 20 & 35 & 45 & 40 & 40 & 45 & $\mathrm{r}$ & $\mathrm{r}$ \\
400 & 400 & 400 & 400 & 200 & 200 & 200 & 200 & 150 & 150 & 400 & 400 & 150 & 200 & 150 & 400 & 400 & 200 & 200 & 400 & $\mathrm{e}$ & $\mathrm{e}$ \\
100 & 100 & 100 & 98 & 100 & 100 & 100 & 100 & 100 & 100 & 100 & 100 & 100 & 100 & 100 & 100 & 100 & 100 & 100 & 100 & $\mathrm{~s}$ & $\mathrm{q}$ \\
\hline
\end{tabular}

subassociation corallorhizetosum trifidae

$\begin{array}{rrllllllllllllllllllll}2 & + & + & + & 1 & 1 & 1 & 1 & 2 & 1 & 2 & 1 & 2 & 2 & 1 & . & . & . & . & . & 26 & 84 \\ . & + & + & + & + & + & + & + & 1 & 1 & . & + & 2 & 1 & + & + & 1 & 1 & + & . & 24 & 77 \\ 1 & 1 & . & \mathrm{r} & . & . & . & . & . & . & . & . & . & . & . & 1 & + & . & + & 1 & 14 & 45 \\ + & + & + & . & + & + & . & . & . & . & + & . & . & . & . & . & . & . & . & . & 13 & 42 \\ . & + & + & . & + & . & . & + & . & . & + & + & . & . & . & . & + & + & . & . & 14 & 45 \\ . & \mathrm{r} & . & . & + & . & . & . & . & . & . & . & . & . & 2 & . & . & . & . & . & 4 & 13 \\ + & . & \mathrm{r} & + & + & . & + & . & + & . & + & . & + & + & + & + & 1 & + & . & + & 20 & 65 \\ . & \mathrm{r} & . & \mathrm{r} & . & + & . & + & + & + & + & + & + & . & + & . & . & . & . & . & 16 & 52 \\ + & . & + & 1 & + & + & . & . & + & . & . & + & . & . & . & . & . & . & . & . & 10 & 32 \\ . & + & . & . & + & . & . & . & . & + & . & . & + & . & . & . & + & . & . & . & 11 & 35\end{array}$

\section{nion sylvaticae}

\begin{tabular}{|cccccc|}
+ & + & + & + & + & + \\
+ & + & + & + & + & $\cdot$ \\
+ & + & + & + & + & + \\
+ & + & 1 & + & + & + \\
+ & $\cdot$ & + & $\cdot$ & + & $\cdot$ \\
$\cdot$ & + & + & + & $\cdot$ & + \\
\hline
\end{tabular}

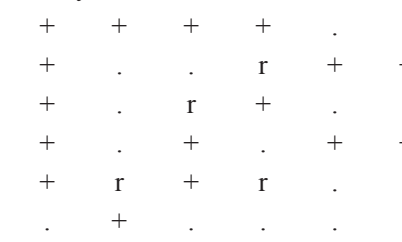




\begin{tabular}{|c|c|c|c|c|c|c|c|c|c|c|c|c|c|}
\hline & & Relevé number & 1 & 2 & 3 & 4 & 5 & 6 & 7 & $8^{*}$ & 9 & 10 & 11 \\
\hline Ch scap & Europ.-Caucas. & Stellaria holostea subsp. holostea & . & . & . & + & . & . & . & . & . & . & . \\
\hline P caesp & Orof. S Europ. & Laburnum alpinum & . & . & . & . & + & . & + & + & . & + & . \\
\hline H caesp & Europ.-Caucas. & Festuca heterophylla & . & . & . & . & . & . & . & . & . & . & . \\
\hline $\mathrm{NP}$ & Eurosib. & Ribes alpinum & . & . & . & + & . & . & . & . & . & . & . \\
\hline G bulb & Eurosib. & Gagea lutea & . & . & . & . & . & . & . & . & . & . & . \\
\hline P caesp & Orof. S Europ. & Lonicera alpigena subsp. alpigena & . & . & . & . & . & . & . & . & . & . & . \\
\hline G rhiz & Europ.-Caucas. & Mercurialis perennis & . & . & . & . & $\mathrm{r}$ & . & . & . & . & . & . \\
\hline G rhiz & C Europ. & Euphorbia dulcis & . & . & . & . & . & . & + & + & . & . & . \\
\hline P caesp & Europ. & Sorbus aucuparia subsp. aucuparia & . & . & . & . & . & . & . & + & . & . & . \\
\hline G rhiz & Circumbor. & Polygonatum odoratum & . & . & . & . & . & . & . & . & . & . & + \\
\hline H scap & Paleotemp. & Heracleum sphondylium subsp. ternatum & . & . & . & . & . & . & . & . & . & . & . \\
\hline T rept & Cosmopol. & Stellaria media subsp. media & . & . & . & . & . & . & . & . & . & . & . \\
\hline G rhiz & Orof. SE-Europ. & Doronicum columnae & . & . & . & . & . & . & . & . & . & . & . \\
\hline \multirow[t]{2}{*}{ P scap } & Europ.-Caucas. & Acer pseudoplatanus & . & . & . & . & 1 & . & . & . & . & . & . \\
\hline & & \multicolumn{12}{|c|}{ Transgressive species from the order Quercetalia pubescenti-petraeae } \\
\hline H scap & Endem. & Digitalis lutea subsp. australis & . & . & . & . & $\mathrm{r}$ & . & . & . & . & . & . \\
\hline G rhiz & Paleotemp. & Epipactis helleborine (s.1.) & . & . & . & . & . & + & . & . & . & $\cdot$ & . \\
\hline P caesp & Paleotemp. & Sorbus aria subsp. aria & . & . & . & . & . & . & . & . & + & . & . \\
\hline G rhiz & Circumbor. & Hepatica nobilis & . & . & . & + & . & . & . & . & . & . & . \\
\hline $\mathrm{H}$ ros & Eurimedit. & Viola alba subsp. dehnhardtii & . & . & + & . & . & . & . & . & . & . & . \\
\hline $\mathrm{H}$ ros & Eurimedit. & Silene italica subsp. italica & . & + & . & . & . & . & . & . & . & . & . \\
\hline G Par & Circumbor. & Monotropa hypopitys & . & . & . & . & . & . & . & . & . & . & . \\
\hline \multirow[t]{2}{*}{$\mathrm{H}$ caesp } & Euromedit. & Poa sylvicola & . & . & . & . & . & . & . & . & . & . & . \\
\hline & & \multicolumn{12}{|c|}{ Charact. species of the class Querco-Fagetea } \\
\hline NP & N Eurimedit. & Rubus hirtus & 1 & + & 1 & + & . & . & $\mathrm{r}$ & + & $\mathrm{r}$ & $\mathrm{r}$ & . \\
\hline H ros & Eurimedit. & Potentilla micrantha & . & + & + & + & $\mathrm{r}$ & . & . & . & . & $\mathrm{r}$ & . \\
\hline G rhiz & Eurimedit. & Cephalanthera damasonium & + & + & . & . & $\mathrm{r}$ & . & $\mathrm{r}$ & + & $\mathrm{r}$ & + & + \\
\hline G bulb & Europ.-Caucas. & Galanthus nivalis subsp. nivalis & . & 1 & . & . & 1 & . & . & + & + & . & + \\
\hline H rept & Europ.-Caucas. & Ajuga reptans & . & . & + & . & . & . & $\mathrm{r}$ & . & + & + & + \\
\hline H scap & Paleotemp. & Sanicula europaea & . & + & . & . & . & . & . & . & + & + & + \\
\hline H scap & Eurosib. & Hieracium murorum & . & . & . & + & $\mathrm{r}$ & . & . & . & . & . & + \\
\hline H caesp & Paleotemp. & Melica uniflora & . & . & . & . & 1 & + & $\mathrm{r}$ & . & + & $\mathrm{r}$ & . \\
\hline H scap & Eurasiat. & Cruciata glabra subsp. glabra & . & . & . & . & . & . & . & . & . & . & . \\
\hline \multirow[t]{2}{*}{ H scap } & Europ.-Caucas. & Hieracium racemosum & . & . & . & . & . & . & + & + & . & . & . \\
\hline & & Accompanying taxa & & & & & & & & & & & \\
\hline T scap & Eurasiat. & Galium aparine & . & + & + & + & . & . & . & . & . & . & . \\
\hline H scap & Paleotemp. & Alliaria petiolata & . & . & . & . & . & . & . & . & . & . & . \\
\hline H scap & S Europ.-Sudsib. & Anthriscus nemorosa & . & . & 1 & . & . & . & . & . & . & . & . \\
\hline H scap & Eurasiat. & Scrophularia scopolii & . & . & . & . & . & . & . & . & . & . & . \\
\hline T scap & Paleotemp. & Lapsana communis & . & . & . & . & . & . & . & . & . & . & . \\
\hline \multirow[t]{2}{*}{ G bulb } & W Europ. & Bunium bulbocastanum & . & . & . & . & . & . & . & . & . & . & . \\
\hline & & Sporadic taxa & 0 & 0 & 0 & 2 & 1 & 1 & 2 & 0 & 0 & 2 & 1 \\
\hline
\end{tabular}




\begin{tabular}{|c|c|c|c|c|c|c|c|c|c|c|c|c|c|c|c|c|c|c|c|c|c|}
\hline 12 & 13 & 14 & 15 & 16 & 17 & 18 & 19 & 20 & 21 & 22 & 23 & 24 & 25 & 26 & 27 & 28 & 29 & 30 & 31 & Pres & Freq \\
\hline . & . & . & $\mathrm{r}$ & . & . & . & . & $\cdot$ & . & . & + & . & & 1 & & . & . & . & + & 5 & $\overline{16}$ \\
\hline . & . & & . & . & . & . & . & . & & . & . & . & 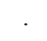 & . & . & & . & . & . & 4 & 13 \\
\hline . & . & . & $\mathrm{r}$ & & . & . & . & . & . & . & & + & . & . & . & . & . & + & + & 4 & 13 \\
\hline+ & $\mathrm{r}$ & & . & . & . & . & . & . & & . & . & . & . & & . & & . & . & 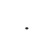 & 3 & 10 \\
\hline+ & + & + & & & & & . & & . & & & & & . & & . & & & & 3 & 10 \\
\hline+ & . & & . & . & . & . & . & . & & . & . & . & . & & + & & . & . & + & 3 & 10 \\
\hline . & . & & $\mathrm{r}$ & . & . & . & . & . & . & . & . & . & . & . & . & . & . & . & 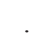 & 2 & 6 \\
\hline & . & . & . & & . & . & . & . & . & . & & & . & . & . & . & . & . & & 2 & 6 \\
\hline . & . & + & . & . & . & . & . & . & . & . & . & . & . & . & . & . & . & . & 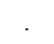 & 2 & 6 \\
\hline . & . & & . & . & . & . & . & . & . & . & . & . & . & . & . & . & . & . & + & 2 & 6 \\
\hline . & . & $\mathrm{r}$ & . & . & . & . & & . & & . & . & . & . & & 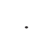 & & . & . & + & 2 & 6 \\
\hline & . & . & . & & & . & . & . & + & & & & . & . & . & . & + & & & 2 & 6 \\
\hline . & . & & . & . & . & . & . & . & . & . & . & + & & + & . & . & . & . & . & 2 & 6 \\
\hline . & . & . & & . & . & & . & & . & & & & & . & & & & & & 1 & 3 \\
\hline
\end{tabular}
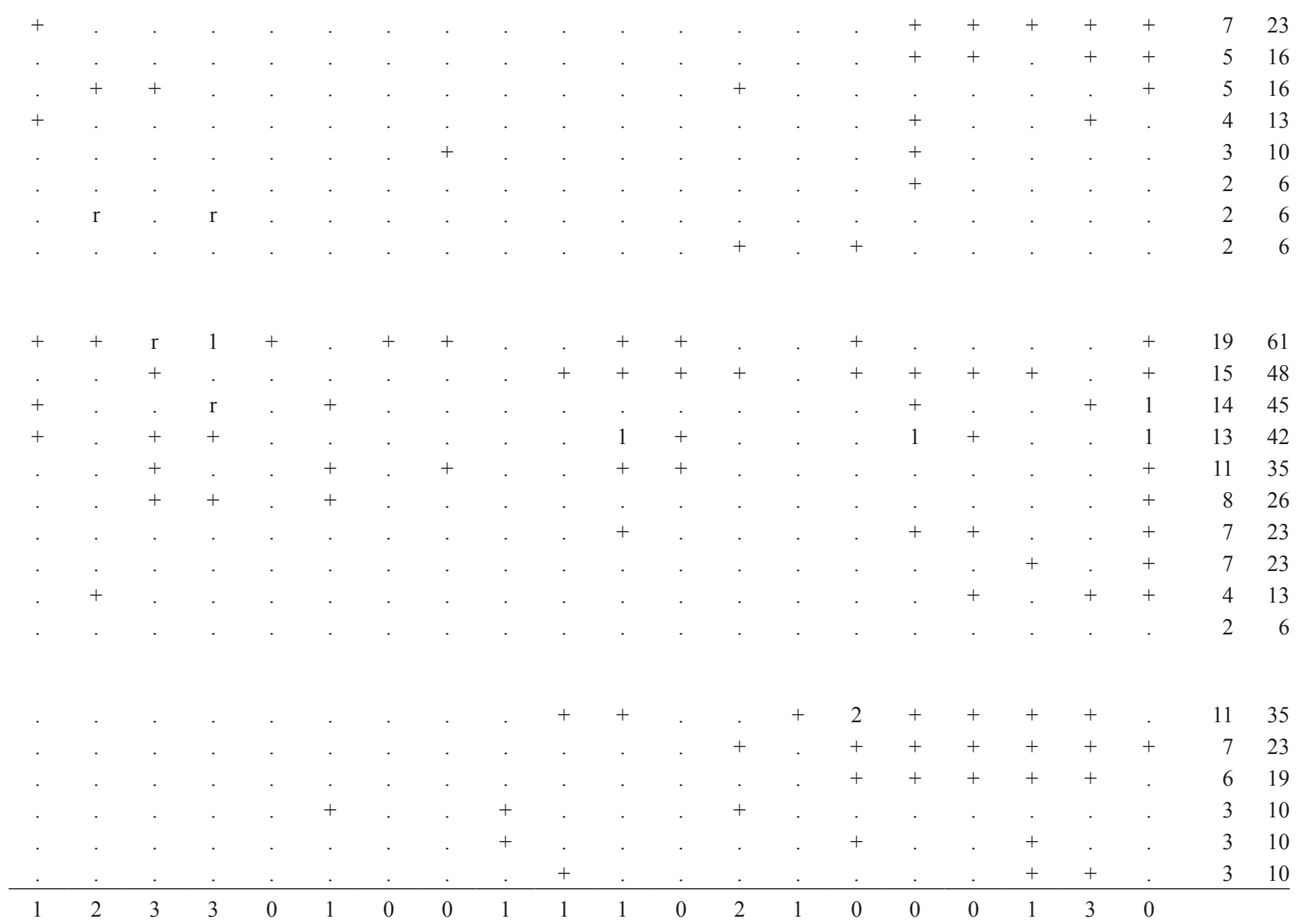
Table 11: Impoverished facies of the association Cardamino kitaibelii-Fagetum sylvaticae Ubaldi, Zanotti, Puppi, Speranza et Corbetta ex Ubaldi 1993.

Tabela 11: Osiromašeni facies asociacije Cardamino kitaibelii-Fagetum sylvaticae Ubaldi, Zanotti, Puppi, Speranza et Corbetta ex Ubaldi 1993.

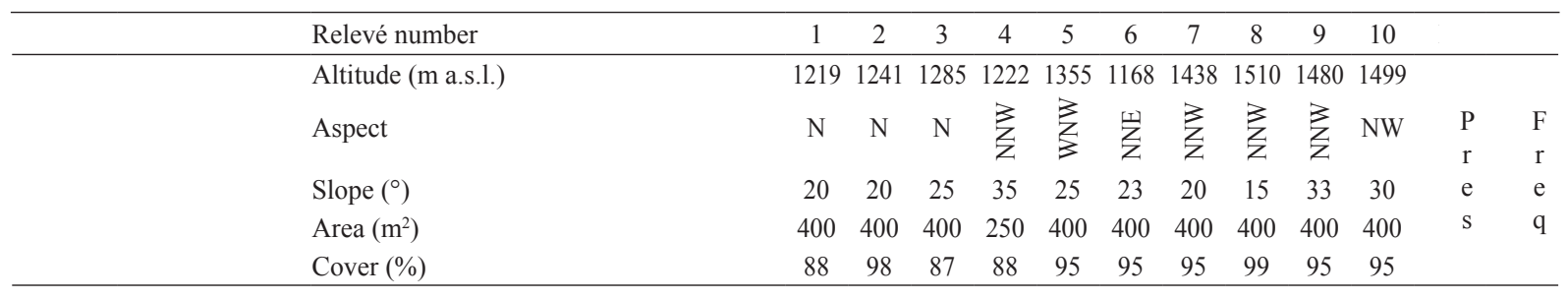

Diagnostic species of the impoverished facies of the association Cardamino kitaibelii-Fagetum sylvaticae

$\mathrm{H}$ caesp Subatl. $\quad$ Brachypodium rupestre $\quad 2 \quad 1 \quad 2 \quad 1+t_{+}++_{+}^{+}+10$

P caesp S Europ.-Sudsib. Laburnum anagyroides subsp. anagyroides $++++\quad+\quad$. . . $\quad+\quad$. $\quad 50$

Charact. species of the alliance Aremonio-Fagion sylvaticae* and diff. of the sub-all. Cardamino kitaibelii-

\section{Fagenion sylvaticae}

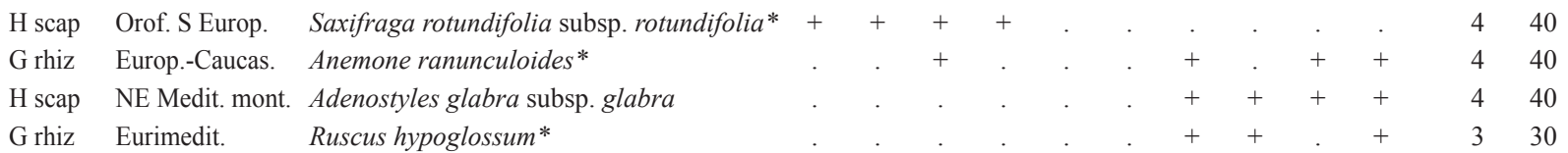

Charact. species of the order Fagetalia sylvaticae

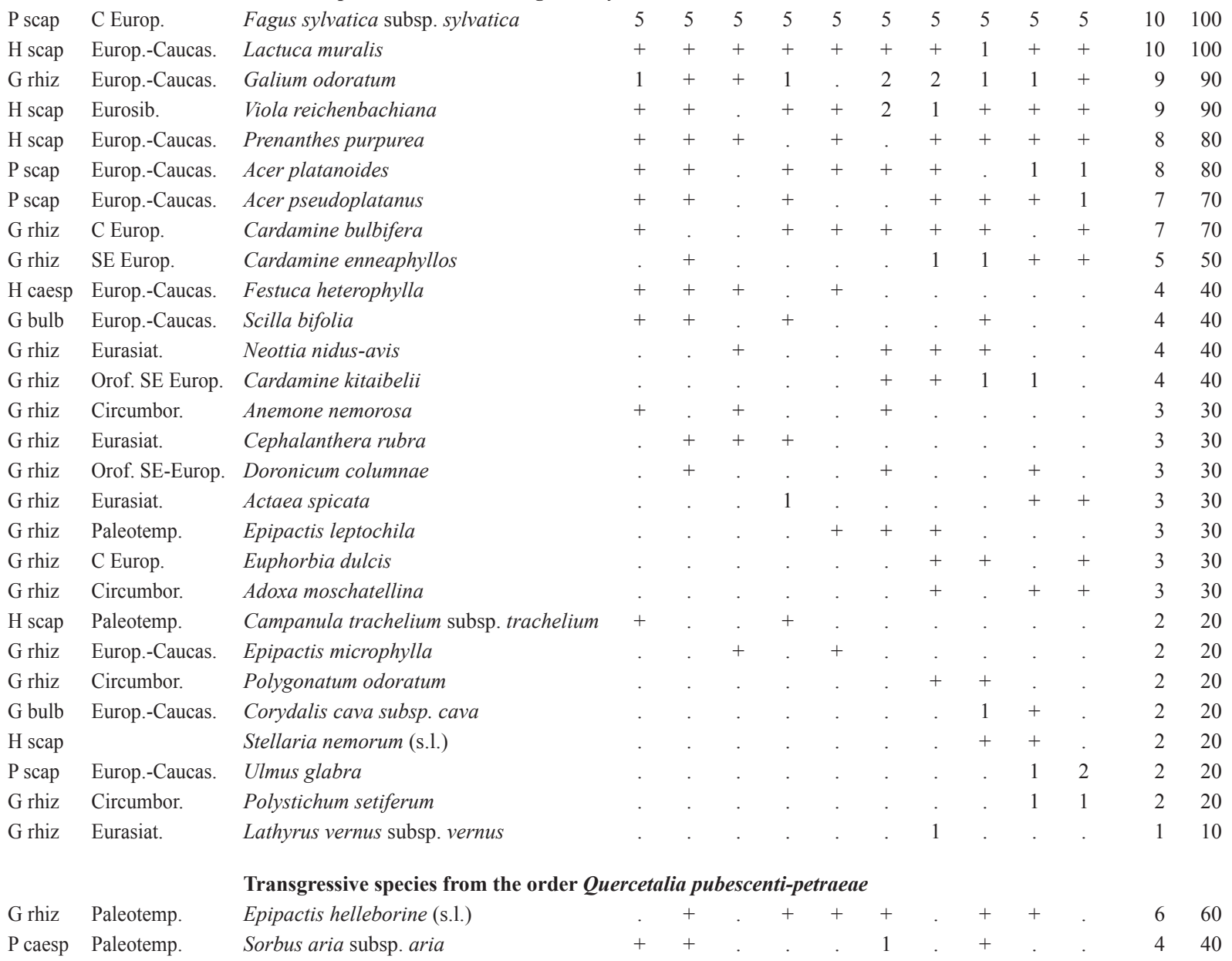




\begin{tabular}{|c|c|c|c|c|c|c|c|c|c|c|c|c|c|c|}
\hline & & Relevé number & 1 & 2 & 3 & 4 & 5 & 6 & 7 & 8 & 9 & 10 & Pres & Freq \\
\hline P scap & S Europ.-Sudsib. & Fraxinus ornus subsp. ornus & + & . & . & . & . & + & + & . & + & . & 4 & 40 \\
\hline $\mathrm{H}$ ros & Eurimedit. & Silene italica subsp. italica & + & + & + & . & . & . & . & . & . & . & 3 & 30 \\
\hline P scap & SE Europ. & Acer opalus subsp. obtusatum & + & . & + & . & . & . & . & . & . & . & 2 & 20 \\
\hline H scap & Endem. & Digitalis lutea subsp. australis & . & + & . & + & . & . & . & . & . & . & 2 & 20 \\
\hline \multirow[t]{2}{*}{ G bulb } & Orof. C Europ. & Lilium bulbiferum subsp. croceum & . & . & . & . & . & . & + & . & . & + & 2 & 20 \\
\hline & & Character species of the class Querco-Fa & etea & & & & & & & & & & & \\
\hline H scap & Europ.-Caucas. & Hieracium racemosum & + & 1 & 1 & . & + & . & + & + & . & . & 6 & 60 \\
\hline P caesp & Subatlant. & Daphne laureola & + & + & . & + & . & + & + & + & . & . & 6 & 60 \\
\hline H scap & Eurosib. & Hieracium murorum & + & 1 & 2 & . & . & + & . & . & . & . & 4 & 40 \\
\hline NP & N Eurimedit. & Rubus hirtus & . & . & . & . & . & . & + & + & + & + & 4 & 40 \\
\hline H caesp & Orof. SE Europ. & Luzula sylvatica subsp. sylvatica & 1 & 1 & + & . & . & . & . & . & . & . & 3 & 30 \\
\hline H scap & Paleotemp. & Sanicula europaea & + & . & . & . & + & . & . & + & . & . & 3 & 30 \\
\hline G bulb & Europ.-Caucas. & Galanthus nivalis subsp. nivalis & . & + & . & + & . & . & . & . & . & . & 2 & 20 \\
\hline H caesp & Paleotemp. & Melica uniflora & . & + & . & . & . & . & + & . & . & . & 2 & 20 \\
\hline H scap & Circumbor. & Solidago virgaurea subsp. virgaurea & . & + & . & . & . & . & + & . & . & . & 2 & 20 \\
\hline H rept & Europ.-Caucas. & Ajuga reptans & . & . & . & . & . & . & + & + & . & . & 2 & 20 \\
\hline \multirow[t]{2}{*}{ G rhiz } & S Europ.-Sudsib. & Lathyrus venetus & . & . & . & . & . & . & + & + & . & . & 2 & 20 \\
\hline & & Accompanying taxa & & & & & & & & & & & & \\
\hline $\mathrm{H}$ bienn & S Europ.-Sudsib. & Arabis turrita & + & + & + & . & . & . & . & . & . & . & 3 & 30 \\
\hline NP & Circumbor. & Rubus idaeus & + & . & . & + & . & . & . & . & . & . & 2 & 20 \\
\hline H scap & Sudsib. & Veronica chamaedrys subsp. chamaedrys & . & . & . & . & . & . & . & . & + & + & 2 & 20 \\
\hline \multirow[t]{2}{*}{ NP } & Eurasiat. & Rosa canina & . & . & . & . & . & . & . & . & + & + & 2 & 20 \\
\hline & & Sporadic taxa & 2 & 2 & 4 & 1 & 0 & 3 & 2 & 2 & 2 & 3 & & \\
\hline
\end{tabular}

Table 12 (Tabela 12): Aceretum obtusati-pseudoplatani Biondi, Casavecchia, Pinzi, Allegrezza et Baldoni 2002.

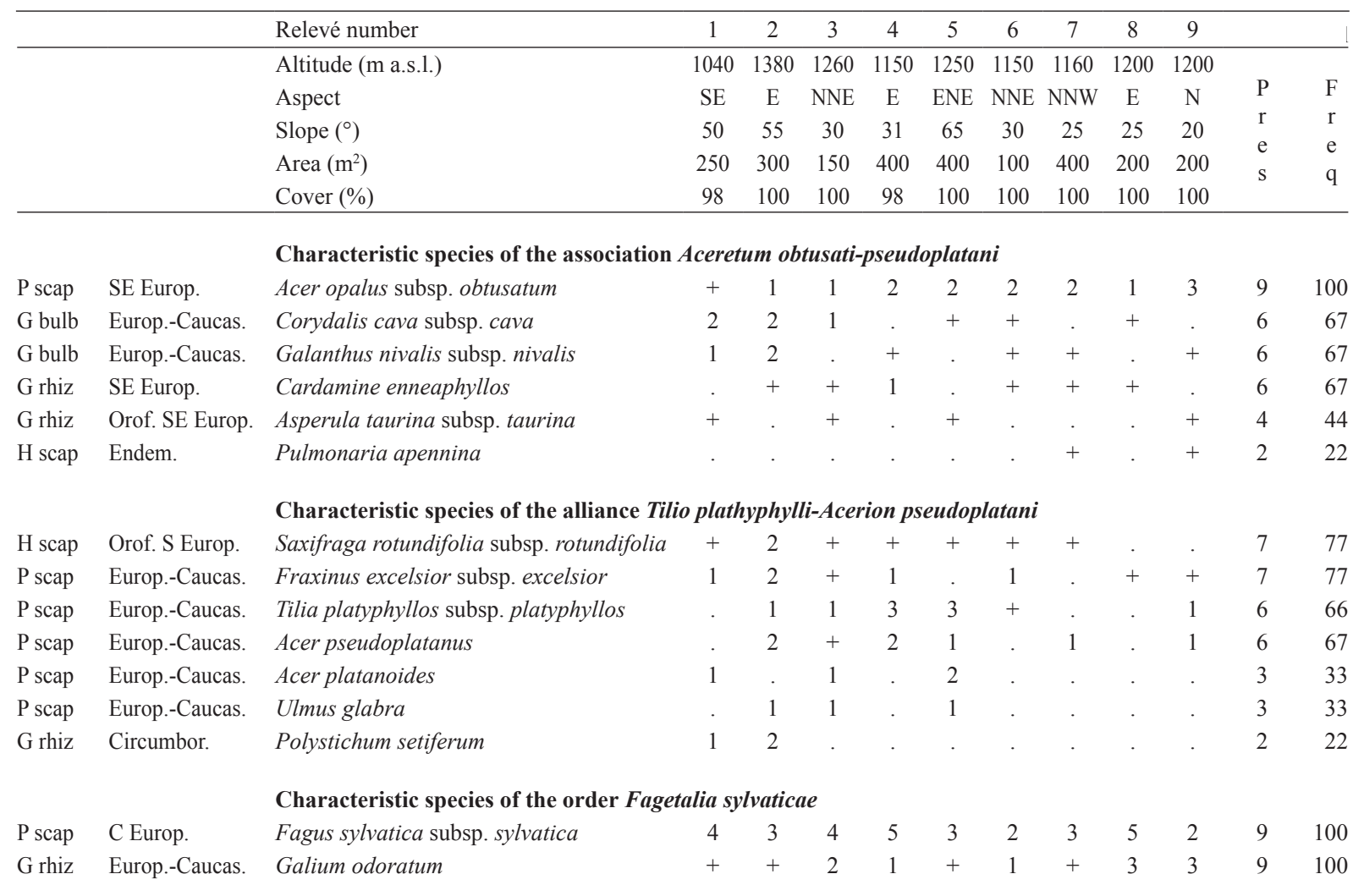




\begin{tabular}{|c|c|c|c|c|c|c|c|c|c|c|c|c|c|}
\hline & & Relevé number & 1 & 2 & 3 & 4 & 5 & 6 & 7 & 8 & 9 & Pres & Freq \\
\hline H caesp & Europ.-Caucas. & Festuca heterophylla & + & + & + & + & + & + & 1 & + & + & 9 & 100 \\
\hline H scap & Europ.-Caucas. & Lactuca muralis & + & + & + & + & . & + & 1 & + & + & 8 & 89 \\
\hline H caesp & Paleotemp. & Melica uniflora & + & 2 & + & . & + & 2 & 1 & 1 & 1 & 8 & 89 \\
\hline G rhiz & Europ.-Caucas. & Mercurialis perennis & $\mathrm{r}$ & + & + & 2 & 2 & . & + & 1 & . & 7 & 78 \\
\hline G rhiz & Orof. SE-Europ. & Doronicum columnae & $\mathrm{r}$ & 1 & . & + & 1 & + & + & + & . & 7 & 78 \\
\hline H scap & Eurosib. & Viola reichenbachiana & + & + & + & . & . & . & + & + & + & 6 & 67 \\
\hline NP & Eurosib. & Ribes alpinum & + & . & . & + & . & 1 & + & + & + & 6 & 67 \\
\hline G rhiz & Europ.-Caucas. & Anemone ranunculoides & + & . & . & + & . & + & + & + & + & 6 & 67 \\
\hline H scap & Paleotemp. & Campanula trachelium subsp. trachelium & . & . & . & 1 & 1 & + & 1 & + & + & 6 & 67 \\
\hline G rhiz & C Europ. & Arum maculatum & + & + & + & . & . & . & + & . & + & 5 & 56 \\
\hline G rhiz & C Europ. & Cardamine bulbifera & + & 1 & . & 1 & . & . & + & + & . & 5 & 56 \\
\hline G bulb & Europ.-Caucas. & Scilla bifolia & + & . & + & . & + & . & + & + & . & 5 & 56 \\
\hline T scap & Subcosmop. & Geranium robertianum & + & . & 1 & . & . & 1 & . & + & 1 & 5 & 56 \\
\hline G rhiz & C Europ. & Euphorbia dulcis & $\mathrm{r}$ & . & . & + & + & . & + & . & . & 4 & 44 \\
\hline G rhiz & Circumbor. & Adoxa moschatellina & . & + & + & . & . & . & . & + & + & 4 & 44 \\
\hline P scap & Paleotemp. & Taxus baccata & . & . & . & . & 1 & 2 & 3 & . & + & 4 & 44 \\
\hline H scap & & Stellaria nemorum (s.1.) & 1 & . & 2 & + & . & . & . & . & . & 3 & 33 \\
\hline H caesp & Circumbor. & Poa nemoralis subsp. nemoralis & . & + & . & . & . & + & + & . & . & 3 & 33 \\
\hline G bulb & Eurosib. & Gagea lutea & . & . & + & + & . & . & . & + & . & 3 & 33 \\
\hline G rhiz & Circumbor. & Anemone nemorosa & . & . & . & + & . & + & + & . & . & 3 & 33 \\
\hline G rhiz & Eurasiat. & Neottia nidus-avis & . & . & . & + & . & + & . & + & . & 3 & 33 \\
\hline P scap & Europ.-Caucas. & Acer campestre & . & . & . & . & . & + & + & . & 1 & 3 & 33 \\
\hline G rhiz & C e S Europ. & Epipactis muelleri & . & . & . & . & . & + & . & + & + & 3 & 33 \\
\hline G rhiz & Orof. SE Europ. & Cardamine kitaibelii & + & + & . & . & . & . & . & . & . & 2 & 22 \\
\hline H scap & NE Medit. mont. & Adenostyles glabra subsp. glabra & $\mathrm{r}$ & . & + & . & . & . & . & . & . & 2 & 22 \\
\hline G bulb & C Europ. & Corydalis pumila & . & + & + & . & . & . & . & . & . & 2 & 22 \\
\hline H scap & Europ.-Caucas. & Prenanthes purpurea & . & + & . & + & . & . & . & . & . & 2 & 22 \\
\hline G bulb & Eurasiat. & Lilium martagon & . & + & . & + & . & . & . & . & . & 2 & 22 \\
\hline G rhiz & Eurasiat. & Polygonatum multiflorum & . & . & + & . & . & . & . & . & + & 2 & 22 \\
\hline G rhiz & Circumbor. & Polygonatum odoratum & . & . & . & + & + & . & . & . & . & 2 & 22 \\
\hline G rhiz & Europ.-Caucas. & Epipactis microphylla & . & . & . & . & . & . & . & + & + & 2 & 22 \\
\hline
\end{tabular}

$\begin{array}{lll}\text { G rhiz } & & \text { Epipactis helleborine (s.1.) } \\ \text { H scap } & \text { Endem. } & \text { Digitalis lutea } \text { subsp. australis } \\ \text { G rhiz } & \text { Circumbor. } & \text { Hepatica nobilis } \\ \text { P caesp } & \text { Paleotemp. } & \text { Sorbus aria subsp. aria } \\ \text { P caesp } & \text { Medit. mont. } & \text { Euonymus latifolius } \\ \text { P scap } & \text { SE Europ. } & \text { Ostrya carpinifolia } \\ \text { P caesp } & \text { S Europ.-Sudsib. } & \text { Euonymus verrucosus } \\ & & \text { Melittis melissophyllum subsp. } \\ \text { H scap } & \text { C Europ. } & \text { melissophyllum } \\ \text { G bulb } & \text { Orof. C Europ. } & \text { Lilium bulbiferum subsp. croceum } \\ \text { NP } & & \text { Emerus majus } \text { (s.l.) } \\ \text { P caesp } & \text { C Europ. } & \text { Crataegus laevigata } \\ \text { H scap } & \text { Paleotemp. } & \text { Sanicula europaea } \\ \text { H caesp } & \text { Euromedit. } & \text { Poa sylvicola } \\ \text { P caesp } & \text { Eurasiat. } & \text { Euonymus europaeus } \\ \text { P caesp } & \text { S Europ.-Sudsib. } & \text { Laburnum anagyroides subsp. anagyroides } \\ \text { P caesp } & \text { C Europ. } & \text { Viburnum lantana } \\ \text { Ch suffr } & \text { Subatl. } & \text { Helleborus foetidus subsp. foetidus }\end{array}$

\section{cetalia pubescenti-petraeae}




\begin{tabular}{|c|c|c|c|c|c|c|c|c|c|c|c|c|c|}
\hline & & Relevé number & 1 & 2 & 3 & 4 & 5 & 6 & 7 & 8 & 9 & Pres & Freq \\
\hline P lian & Eurimedit. & Hedera helix subsp. helix & + & . & . & 1 & + & . & + & . & + & 5 & 56 \\
\hline G rhiz & S Europ.-Sudsib. & Lathyrus venetus & . & + & + & 1 & . & . & . & 1 & + & 5 & 56 \\
\hline P lian & Europ.-Caucas. & Clematis vitalba & . & . & . & + & + & . & + & + & + & 5 & 56 \\
\hline H caesp & Paleotemp. & $\begin{array}{l}\text { Brachypodium sylvaticum subsp. } \\
\text { sylvaticum }\end{array}$ & $\mathrm{r}$ & + & $\cdot$ & . & + & . & . & $\cdot$ & $\cdot$ & 3 & 33 \\
\hline G bulb & S Europ. & $\begin{array}{l}\text { Cyclamen hederifolium subsp. } \\
\text { hederifolium }\end{array}$ & 1 & . & . & + & + & . & . & . & . & 3 & 33 \\
\hline H scap & Eurasiat. & Aquilegia vulgaris & $\mathrm{r}$ & . & . & . & + & . & . & . & . & 2 & 22 \\
\hline NP & N Eurimedit. & Rubus hirtus & . & + & 1 & . & + & . & . & . & . & 3 & 33 \\
\hline G rhiz & SE Europ. & Anemone apennina subsp. apennina & . & + & + & . & + & . & . & . & . & 3 & 33 \\
\hline H ros & NE Stenomedit. & $\begin{array}{l}\text { Aremonia agrimonoides subsp. } \\
\text { agrimonoides }\end{array}$ & . & . & $\cdot$ & . & . & + & . & 1 & + & 3 & 33 \\
\hline $\mathrm{H}$ caesp & Orof. SE Europ. & Luzula sylvatica subsp. sylvatica & . & . & . & . & 1 & . & . & . & + & 2 & 22 \\
\hline H scap & Circumbor. & Solidago virgaurea subsp. virgaurea & . & . & . & . & + & . & . & . & 1 & 2 & 22 \\
\hline \multirow[t]{2}{*}{$\mathrm{H}$ ros } & Eurimedit. & Potentilla micrantha & . & . & . & . & . & . & . & + & + & 2 & 22 \\
\hline & & Accompanying taxa & & & & & & & & & & & \\
\hline T scap & Eurasiat. & Galium aparine & $\mathrm{r}$ & . & 1 & . & . & + & + & + & . & 5 & 56 \\
\hline H scap & E Medit. mont. & Lamium garganicum subsp. laevigatum & + & . & + & . & . & . & . & + & + & 4 & 44 \\
\hline H caesp & Cosmopol. & Cystopteris fragilis & $\mathrm{r}$ & + & . & . & . & . & + & . & . & 3 & 33 \\
\hline T scap & Eurasiat. & Veronica hederifolia subsp. hederifolia & . & . & + & . & . & + & . & . & + & 3 & 33 \\
\hline H scap & Europ. & Laserpitium latifolium & . & . & . & + & + & . & . & . & + & 3 & 33 \\
\hline H bienn & S Europ.-Sudsib. & Arabis turrita & . & . & . & . & + & . & . & . & + & 2 & 22 \\
\hline NP & Circumbor. & Rubus idaeus & . & . & . & . & . & + & . & + & . & 2 & 22 \\
\hline T scap & Eurasiat. & Chaerophyllum temulum & . & . & . & . & . & + & . & . & 1 & 2 & 22 \\
\hline \multirow[t]{2}{*}{ G bulb } & W Europ. & Bunium bulbocastanum & . & . & . & . & . & . & . & + & + & 2 & 22 \\
\hline & & Sporadic taxa & 9 & 1 & 5 & 0 & 3 & 5 & 5 & 2 & 8 & & \\
\hline
\end{tabular}

Table 13: Scheme of the correlation between shrubberies coenoses and the considered woods syntaxa.

Tabela 13: Shema korelacije med robnimi grmovnimi združbami in obravnavanimi gozdnimi sintaksoni.

\begin{tabular}{|c|c|c|}
\hline & Shrub plant communities & Principal species \\
\hline $\begin{array}{l}\text { Scutellario columnae-Ostryetum carpi- } \\
\text { nifoliae violetosum reichenbachianae }\end{array}$ & $\begin{array}{c}\text { Spartio juncei-Cytisetum sessilifolii } \\
\text { Cytision sessilifolii }\end{array}$ & $\begin{array}{l}\text { Spartium junceum, Cytisophyllum sessilifolius, } \\
\text { Lonicera etrusca, Emerus majus subsp. majus and } \\
\text { Juniperus communis }\end{array}$ \\
\hline $\begin{array}{l}\text { Carici digitatae-Ostryetum carpini- } \\
\text { foliae }\end{array}$ & $\begin{array}{c}\text { Cytiso sessilifolii-Crataegetum laevigatae } \\
\text { Berberidion vulgaris }\end{array}$ & $\begin{array}{l}\text { Cytisophyllum sessilifolius, Crataegus monogyna, } \\
\text { Juniperus communis, Emerus majus subsp. majus } \\
\text { and Rosa canina }\end{array}$ \\
\hline $\begin{array}{l}\text { Lathyro veneti-Fagetum sylvaticae } \\
\text { lathyretosum veneti }\end{array}$ & $\begin{array}{c}\text { Cytiso sessilifolii-Crataegetum laevigatae } \\
\text { Berberidion vulgaris }\end{array}$ & $\begin{array}{l}\text { Crataegus laevigata, Cytisophyllum sessilifolius, } \\
\text { Rhamnus cathartica, Juniperus communis and } \\
\text { Viburnum lantana }\end{array}$ \\
\hline $\begin{array}{l}\text { Lathyro veneti-Fagetum sylvaticae } \\
\text { hieracietosum murori }\end{array}$ & $\begin{array}{l}\text { Grouping Erica arborea and Pteridium aqulinum } \\
\text { Cytision sessilifolii }\end{array}$ & $\begin{array}{l}\text { Erica arborea, Pteridium aquilinum subsp. } \\
\text { aquilinum, Juniperus communis, Cytisophyllum } \\
\text { sessilifolius and Rosa canina }\end{array}$ \\
\hline $\begin{array}{l}\text { Solidagini-Fagetum sylvaticae luzule- } \\
\text { tosum sylvaticae }\end{array}$ & $\begin{array}{c}\text { Grouping Cytisus scoparius subsp. scoparius and } \\
\text { Juniperus communis } \\
\text { Berberidion vulgaris }\end{array}$ & $\begin{array}{l}\text { Crataegus laevigata, Cytisus scoparius subsp. } \\
\text { scoparius, Juniperus communis, Vaccinium myr- } \\
\text { tillusand Erica arborea }\end{array}$ \\
\hline $\begin{array}{l}\text { Cardamino kitaibelii-Fagetum sylvati- } \\
\text { cae anemonetosum nemorosae }\end{array}$ & $\begin{array}{c}\text { Rhamno alpinae-Amelanchieretum ovalis } \\
\text { Berberidion vulgaris }\end{array}$ & $\begin{array}{l}\text { Crataegus laevigata, Rhamnus alpina subsp. } \\
\text { alpina, Rhamnus cathartica, Juniperus communis } \\
\text { and Laburnum alpinum }\end{array}$ \\
\hline $\begin{array}{l}\text { Cardamino kitaibelii-Fagetum sylvati- } \\
\text { cae cardaminetosum kitaibelii }\end{array}$ & $\begin{array}{l}\text { Rhamno alpinae-Amelanchieretum ovalis } \\
\text { Berberidion vulgaris }\end{array}$ & $\begin{array}{l}\text { Rhamnus alpina subsp. alpina, Amelanchier } \\
\text { ovalis subsp. ovalis, Cotoneaster integerrimus, } \\
\text { Juniperus communis and Laburnum alpinum }\end{array}$ \\
\hline $\begin{array}{l}\text { Cardamino kitaibelii-Fagetum sylvati- } \\
\text { cae corallhorizetosum trifidae }\end{array}$ & $\begin{array}{c}\text { Grouping Sorbus aucuparia subsp. aucuparia } \\
\text { and Lonicera alpigena subsp. alpigena } \\
\text { Berberidion vulgaris }\end{array}$ & $\begin{array}{l}\text { Sorbus aucuparia subsp. aucuparia, Rosa pendu- } \\
\text { lina, Juniperus nana, Lonicera alpigena subsp. } \\
\text { alpigena and Laburnum alpinum }\end{array}$ \\
\hline
\end{tabular}

